\title{
Oxidative functionalization of trinor-18 $\alpha$-olean-17(22)-ene derivatives. Annulation of the E-ring by an intramolecular aldol reaction
}

\author{
Kinga Kuczynska, ${ }^{*}$, Jarosław Jaźwiński, ${ }^{a}$ Zbigniew Pakulski, ${ }^{*}, a$ Piotr Cmoch, ${ }^{a}$ Roman Luboradzki ${ }^{b}$ \\ ${ }^{a}$ Institute of Organic Chemistry, Polish Academy of Sciences, Kasprzaka 44/52, 01-224 Warsaw, \\ Poland \\ ${ }^{b}$ Institute of Physical Chemistry, Polish Academy of Sciences, Kasprzaka 44/52, 01-224 Warsaw, \\ Poland
}

\begin{tabular}{|c|c|}
\hline Table of contents & Page \\
\hline Crystal structure determination of compounds 21,23 , and 24 & S2-S5 \\
\hline Key HMBC and NOE correlations & S5 \\
\hline DFT calculation - Figures 1S-11S & S6-S9 \\
\hline DFT calculation - atomic coordinates of the key structures & S9-S51 \\
\hline NMR spectra of compounds $2,8-14,17,19,20,21,23$, and 24 & S52-S79 \\
\hline
\end{tabular}


Single crystal X-ray diffraction measurements were carried out on an Agilent Supernova diffractometer at $100 \mathrm{~K}$ with monochromated $\mathrm{Cu} \mathrm{K} \alpha$ radiation $(1.54184 \mathrm{~A})$. The data reduction was made by using CrysAlisPRO software. ${ }^{1}$ All non-hydrogen atoms were refined as anisotropic while hydrogen atoms were placed in calculated positions and refined in riding mode. CCDC 2055466 (21), CCDC 2031957 (23), CCDC 2031169 (24) contain the supplementary crystallographic data for this paper. These data can be obtained free of charge from The Cambridge Crystallographic Data Centre via www.ccdc.cam.ac.uk/data request/cif

The structures of compounds $\mathbf{2 1}, \mathbf{2 3}$, and $\mathbf{2 4}$ were determined on crystals prepared in a chloroform / methanol solvent system by slow evaporation at room temperature.

Using Olex2, structure 21 was solved with olex2.solve structure solution program using Charge Flipping and refined with olex2.refine refinement package using Gauss-Newton minimisation. ${ }^{2}$ The crystal was of poor quality $[0.09 \times 0.03 \times 0.01 \mathrm{~mm}]$ that a sharp drop in diffraction intensity was observed at high theta angles. Therefore, the measurement was carried out for the theta angle values ranging up to $104.7^{\circ}$. This caused an alert A in the checkCIF report, however the data obtained is absolutely sufficient to determine the correct structure and conformation of the molecule.

The structures $\mathbf{2 3}$ and $\mathbf{2 4}$ were solved by direct methods and refined on $\mathrm{F}^{2}$ by full-matrix least-squares by using SHELXS97 and SHELXL97. ${ }^{3}$ The fragment of molecule 24 was refined as disordered in two parts with occupancy 0.85 and 0.15 respectively.

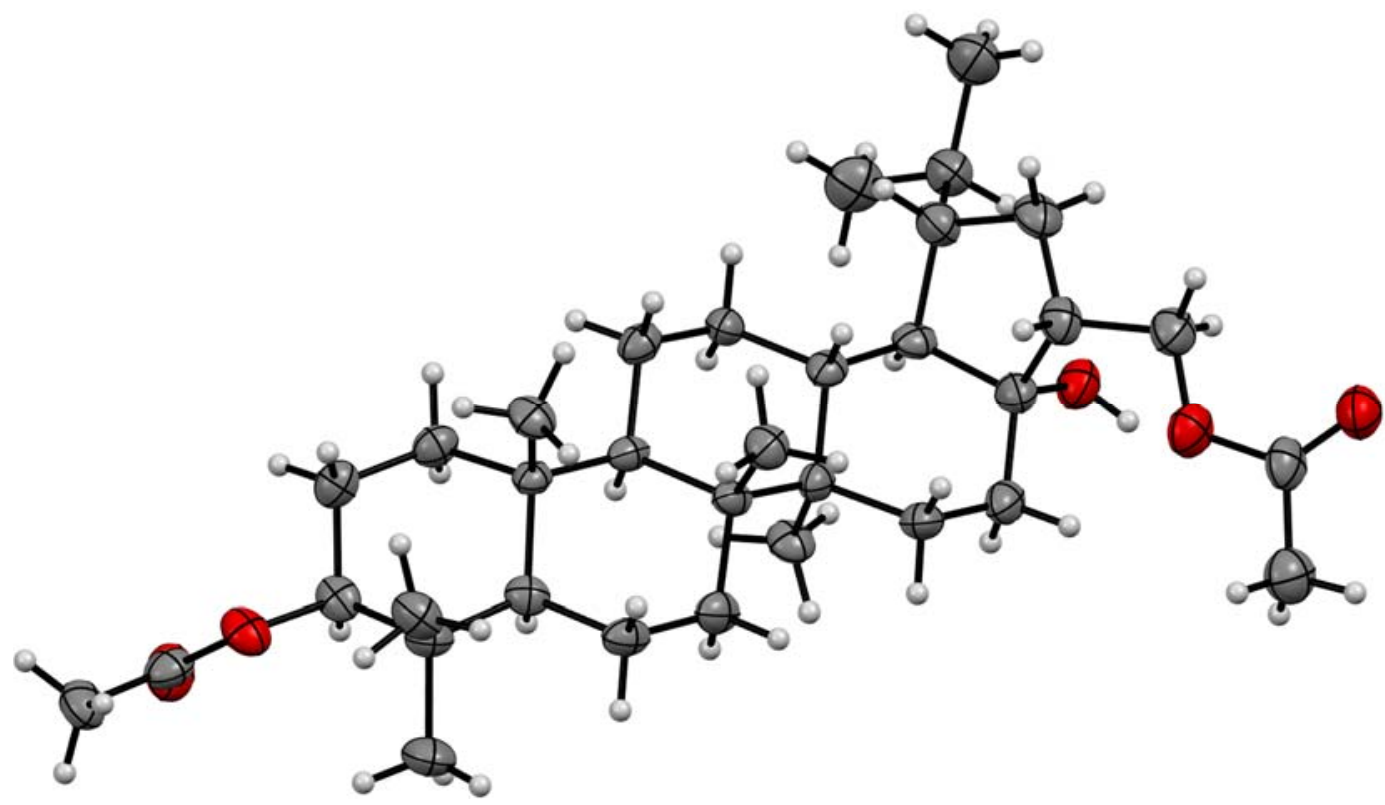

Structure S1. ORTEP diagram of compound 21 (CCDC 2055466). Contour probability level: 50\%.

Table S1. Crystal data for 21.

\begin{tabular}{|l|l|}
\hline Compound & KK768B_010 \\
\hline Empirical formula & $\mathrm{C}_{34} \mathrm{H}_{56} \mathrm{O}_{5}$ \\
\hline Moiety formula & $\mathrm{C}_{34} \mathrm{H}_{56} \mathrm{O}_{5}$ \\
\hline Formula weight & 544.821 \\
\hline CCDC No. & CCDC 2055466 \\
\hline Wavelength & 1.54184 \\
\hline
\end{tabular}

\footnotetext{
${ }^{1}$ Agilent. CrysAlis PRO; Agilent Technologies, Yarnton, England, 2011.

${ }^{2}$ Bourhis, L. J.; Dolomanov, O. V.; Gildea, R. J.; Howard, J. A. K.; Puschmann, H., The anatomy of a comprehensive constrained, restrained refinement program for the modern computing environment - Olex2 dissected, Acta Crystallogr., Sect. A: Found. Crystallogr. 2015, A71, 59-75.

${ }^{3}$ Sheldrick, G. M. A short history of SHELX. Acta Crystallogr., Sect. A: Found. Crystallogr. 2008, A64, 112-122.
} 


\begin{tabular}{|c|c|}
\hline Crystal system & orthorhombic \\
\hline Space group & $P 2_{1} 2_{1} 2_{1}$ \\
\hline \multirow{3}{*}{ Unit cell dimensions } & $a=6.6009(3) \AA$ \\
\hline & $b=13.0155(8) \AA$ \\
\hline & $c=35.547(2) \AA$ \\
\hline Volume & $3054.0(3) \AA^{3}$ \\
\hline $\mathrm{Z}$ & 4 \\
\hline Density Calc. & $1.185 \mathrm{~g} / \mathrm{cm}^{3}$ \\
\hline Absorption coefficient & $0.604 \mathrm{~mm}^{-1}$ \\
\hline $\mathrm{F}(000)$ & 1200 \\
\hline Crystal & Colourless needle \\
\hline Crystal size & $0.09 \times 0.03 \times 0.01 \mathrm{~mm}$ \\
\hline Index ranges & $-6 \leq \mathrm{h} \leq 6,-13 \leq \mathrm{k} \leq 12,-32 \leq 1 \leq 36$ \\
\hline $\begin{array}{l}\text { Reflections collected } \\
\text { (all / independent) }\end{array}$ & $7403 / 3287\left[R_{\text {int }}=0.0545\right]$ \\
\hline Absorption correction & multi-scan \\
\hline Refinement method & Full-matrix least-squares on $F^{2}$ \\
\hline Restraints / parameters & $0 / 362$ \\
\hline Goodness-of-fit on $F^{2}$ & 1.0502 \\
\hline Final $\mathrm{R}$ indices $\left[F^{2}>2 \sigma\left(F^{2}\right)\right]$ & $R_{1}=0.0540, \mathrm{wR}_{2}=0.1214$ \\
\hline$R$ indices (all data) & $R_{1}=0.0715, \mathrm{wR}_{2}=0.1361$ \\
\hline
\end{tabular}

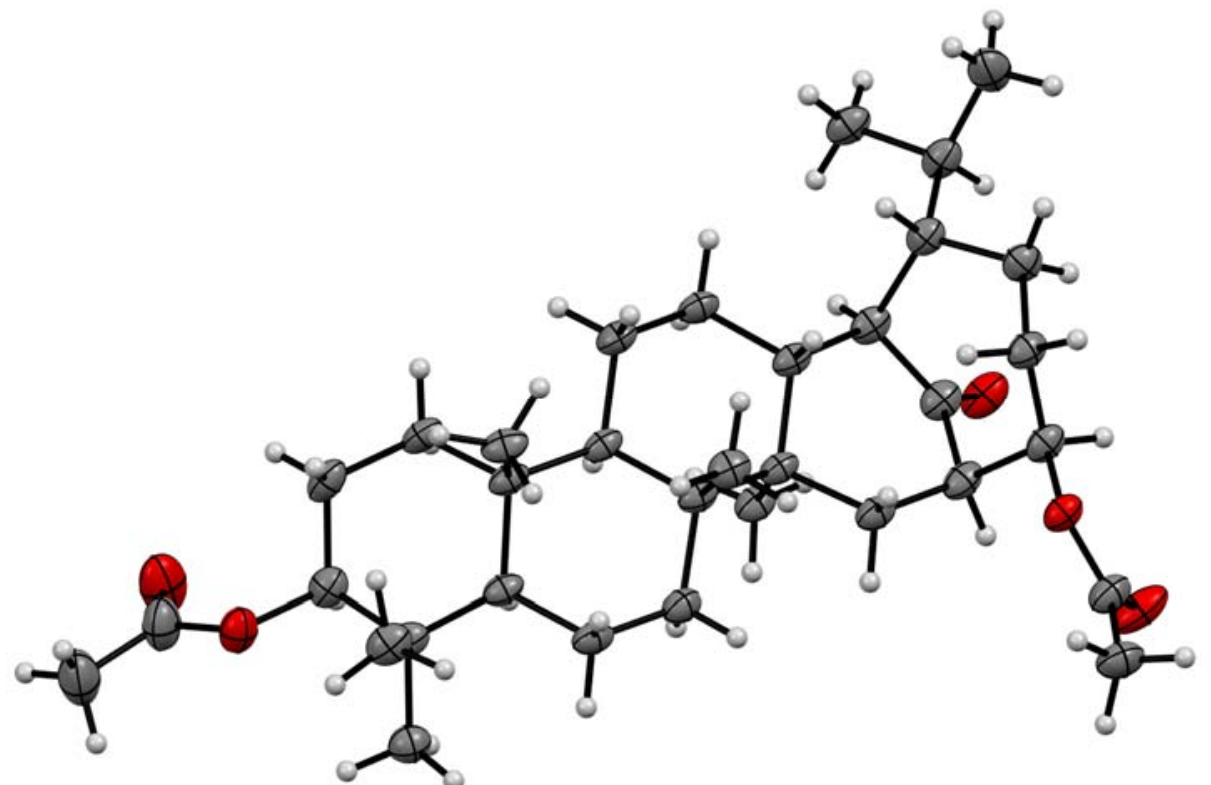

Structure S2. ORTEP diagram of compound 23 (CCDC 2031957). Contour probability level: 50\%.

Table S2. Crystal data for 23.

\begin{tabular}{|c|c|c|}
\hline Compound & \multicolumn{2}{|l|}{$\mathrm{kk} 767 \mathrm{a}$} \\
\hline Empirical formula & \multicolumn{2}{|l|}{$\mathrm{C}_{34} \mathrm{H}_{54} \mathrm{O}_{5}$} \\
\hline Moiety formula & \multicolumn{2}{|l|}{$\mathrm{C}_{34} \mathrm{H}_{54} \mathrm{O}_{5}$} \\
\hline Formula weight & \multicolumn{2}{|l|}{542.77} \\
\hline CCDC No. & \multicolumn{2}{|l|}{ CCDC 2031957} \\
\hline Wavelength & \multicolumn{2}{|l|}{1.54184} \\
\hline Crystal system & \multicolumn{2}{|l|}{ monoclinic } \\
\hline Space group & \multicolumn{2}{|l|}{$P 21$} \\
\hline \multirow{2}{*}{ Unit cell dimensions } & $a=14.7524(6) \AA$ & \\
\hline & $b=7.2005(2) \AA$ & $\beta=101.562(4)^{\circ}$ \\
\hline
\end{tabular}




\begin{tabular}{|l|l|}
\hline & $c=14.8903(6) \AA$ \\
\hline Volume & $1549.62(10)) \AA^{3}$ \\
\hline $\mathrm{Z}$ & 2 \\
\hline Density Calc. & $1.163 \mathrm{~g} / \mathrm{cm}^{3}$ \\
\hline Absorption coefficient & $0.595 \mathrm{~mm}^{-1}$ \\
\hline $\mathrm{F}(000)$ & 596 \\
\hline Crystal & Colorless needle \\
\hline Crystal size & $0.5 \times 0.05 \times 0.01 \mathrm{~mm}$ \\
\hline Index ranges & $-16 \leq \mathrm{h} \leq 18,-8 \leq \mathrm{k} \leq 6,-18 \leq 1 \leq 18$ \\
\hline $\begin{array}{l}\text { Reflections collected } \\
\text { (all / independent) }\end{array}$ & $9447 / 4782\left[R_{\text {int }}=0.0508\right]$ \\
\hline Absorption correction & multi-scan \\
\hline Refinement method & Full-matrix least-squares on $F^{2}$ \\
\hline Restraints / parameters & $1 / 361$ \\
\hline Goodness-of-fit on $F^{2}$ & 1.075 \\
\hline Final R indices $\left[F^{2}>2 \sigma\left(F^{2}\right)\right]$ & $R_{1}=0.0428, \mathrm{wR}_{2}=0.1179$ \\
\hline$R$ indices (all data) & $R_{1}=0.0597, \mathrm{wR}_{2}=0.1242$ \\
\hline
\end{tabular}

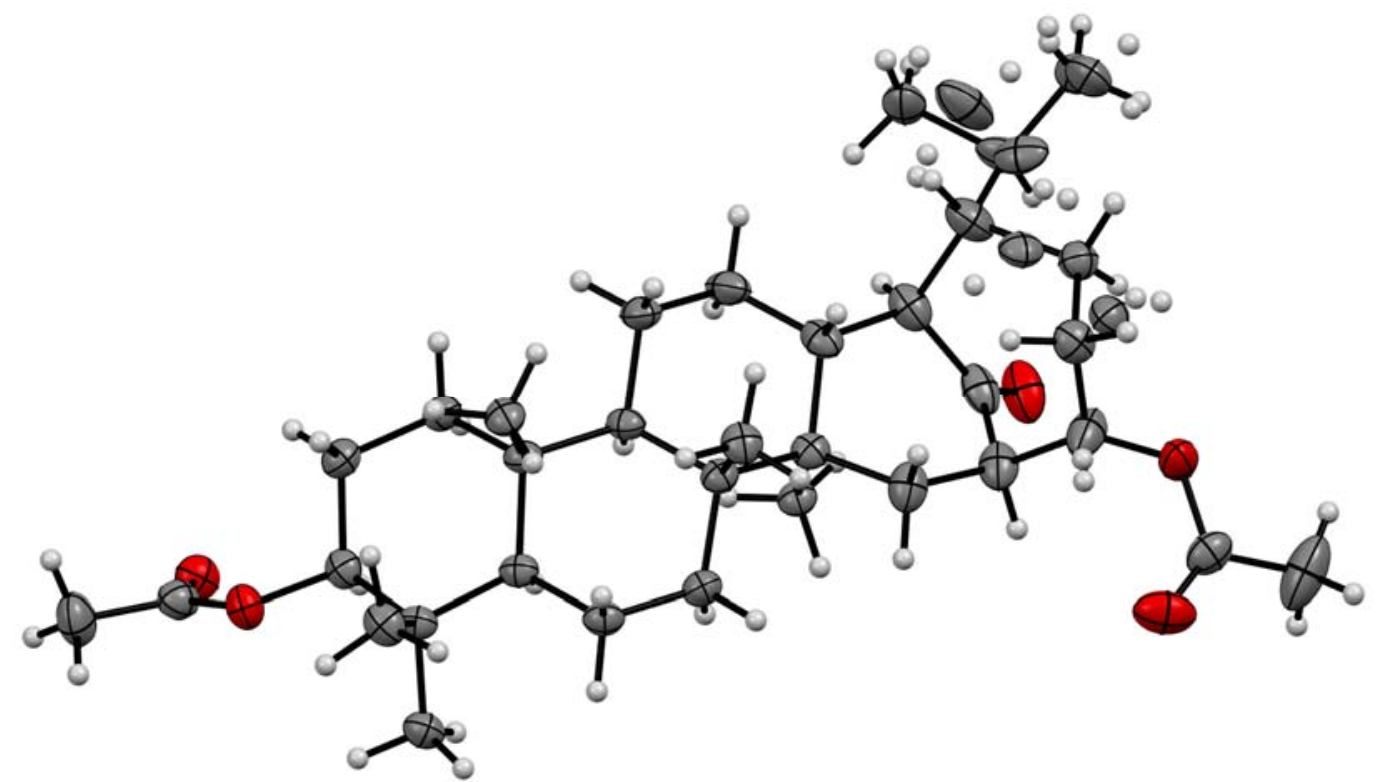

Structure S3. ORTEP diagram of compound 24 (CCDC 2031169). Contour probability level: 50\%.

Table S3. Crystal data for 24.

\begin{tabular}{|l|l|}
\hline Compound & $\mathrm{kk} 767 \mathrm{~b}$ \\
\hline Empirical formula & $\mathrm{C}_{34} \mathrm{H}_{54} \mathrm{O}_{5}$ \\
\hline Moiety formula & $\mathrm{C}_{34} \mathrm{H}_{54} \mathrm{O}_{5}$ \\
\hline Formula weight & 542.77 \\
\hline CCDC No. & $\mathrm{CCDC} 2031169$ \\
\hline Wavelength & 1.54184 \\
\hline Crystal system & orthorhombic \\
\hline Space group & $P 212121$ \\
\hline \multirow{3}{*}{ Unit cell dimensions } & $a=8.12000(10) \AA$ \\
\hline & $b=11.32180(10) \AA$ \\
\hline Volume & $c=32.5914(4) \AA$ \\
\hline Z & $2996.23(6) \AA^{3}$ \\
\hline Density Calc. & 4 \\
\hline
\end{tabular}




\begin{tabular}{|l|l|}
\hline Absorption coefficient & $0.616 \mathrm{~mm}^{-1}$ \\
\hline $\mathrm{F}(000)$ & 1192 \\
\hline Crystal & Colorless plate \\
\hline Crystal size & $0.4 \times 0.3 \times 0.02 \mathrm{~mm}$ \\
\hline Index ranges & $-9 \leq \mathrm{h} \leq 6,-13 \leq \mathrm{k} \leq 13,-38 \leq 1 \leq 39$ \\
\hline $\begin{array}{l}\text { Reflections collected } \\
(\text { all / independent })\end{array}$ & $27660 / 5655\left[R_{\text {int }}=0.0370\right]$ \\
\hline Absorption correction & multi-scan \\
\hline Refinement method & Full-matrix least-squares on $F^{2}$ \\
\hline Restraints / parameters & $0 / 409$ \\
\hline Goodness-of-fit on $F^{2}$ & 1.056 \\
\hline Final R indices $\left[F^{2}>2 \sigma\left(F^{2}\right)\right]$ & $R_{1}=0.0382, \mathrm{wR}_{2}=0.0929$ \\
\hline$R$ indices (all data) & $R_{1}=0.0423, \mathrm{wR}_{2}=0.0943$ \\
\hline
\end{tabular}

Table S4. Key HMBC (------) and NOE (------) correlations.

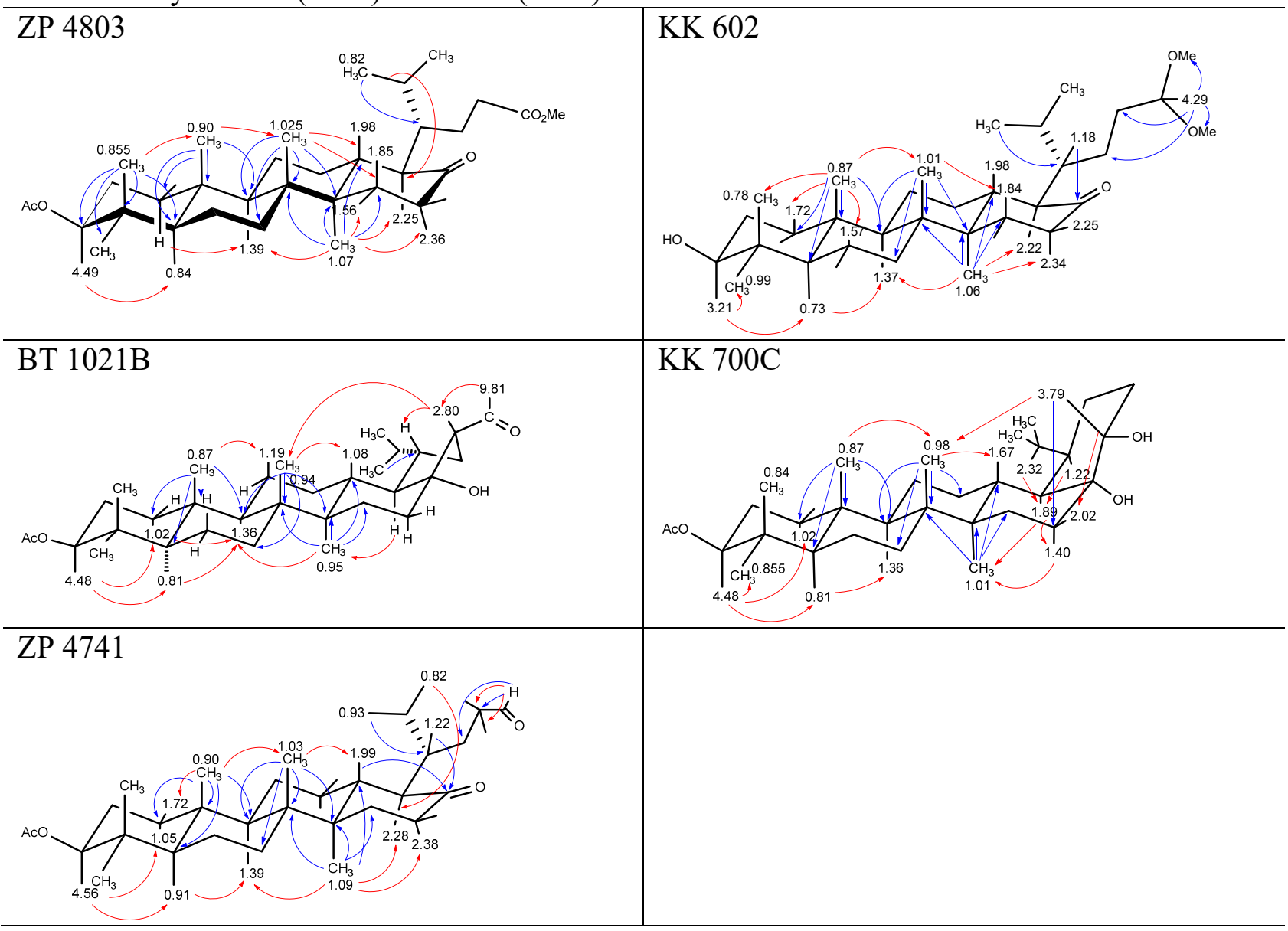




\section{DFT calculations:}

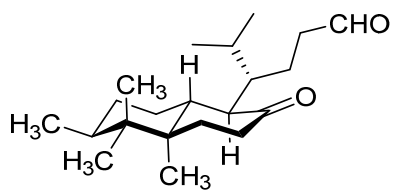

Figure 1S. A simplified structure used in the preliminary calculations

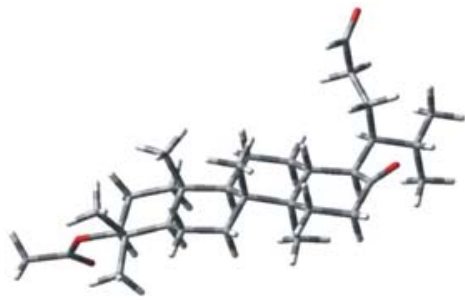

1

$-1552.0802$

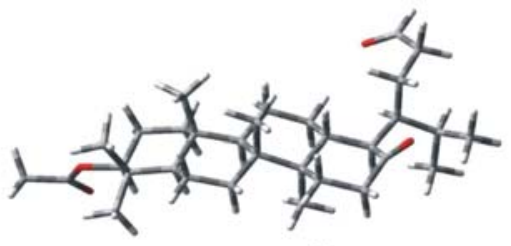

II

$-1552.0797$

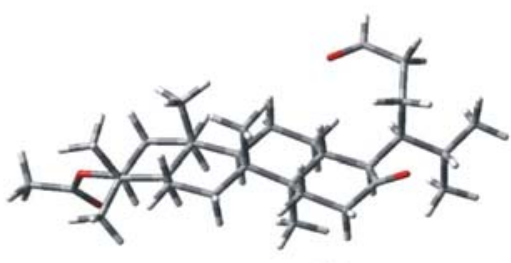

III

Figure $2 \mathrm{~S}$. Three the most populated rotamers of $\mathbf{1 0}(0.22,0.13$, and 0.48 molar fractions, respectively).

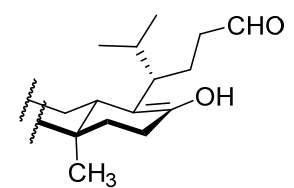

$-1552.0655$

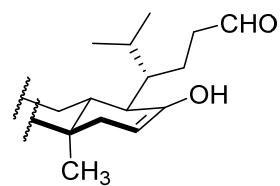

$-1552.0653$

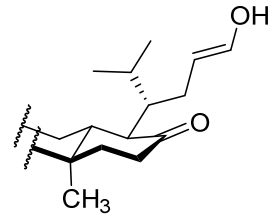

$-1552.0668$

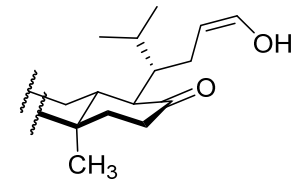

$-1552.0680$

Figure 3S. Enol structures used as input in the calculations; the structures were constructed starting from III (Fig.2S). Boltzmann distribution revealed molar fractions of $0.05,0.05,0.20$, and 0.70 , respectively, if only these four tautomers were considered. However, the molar fractions were much smaller than 0.01 when III was included. Molecular energy differences between the above tautomers and III vary from 34 to $41 \mathrm{~kJ} / \mathrm{mol}$.

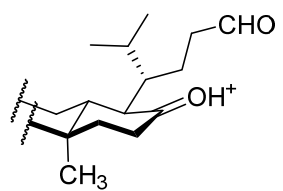

$-1552.4322$

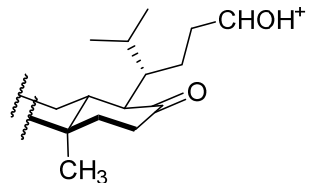

$-1552.4069$

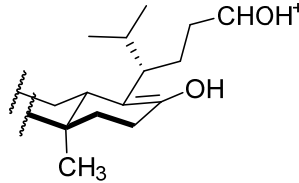

$-1552.3905$

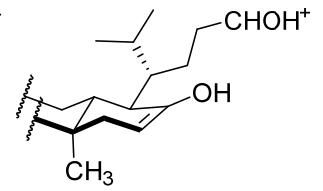

$-1552.3864$

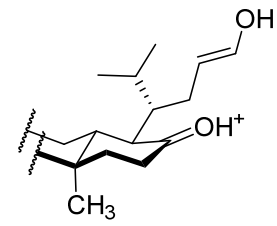

$-1552.4138$

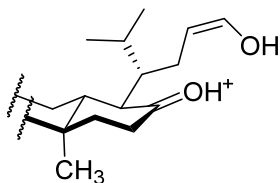

$-1552.4120$

Figure 4S. Molecular energies of protonated compounds; the input structures were constructed starting from III (Fig.2S). The first structure is the most populated $(\mathrm{x} \approx 1)$ 


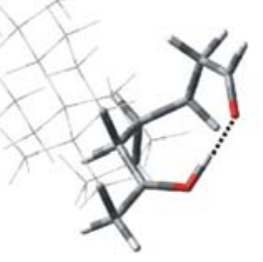

IV

$-1552.4370$

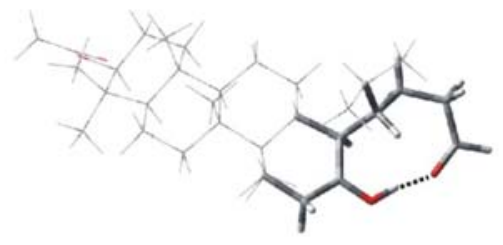

V

$-1552.4458$

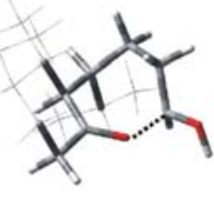

VI

$-1552.4394$

Figure 5S. The rotamers stabilized by hydrogen bonds or the $\mathrm{O} \cdots \mathrm{C}$ interaction (dotted lines). Bond lengths are as

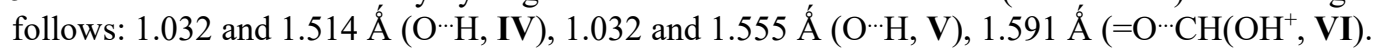

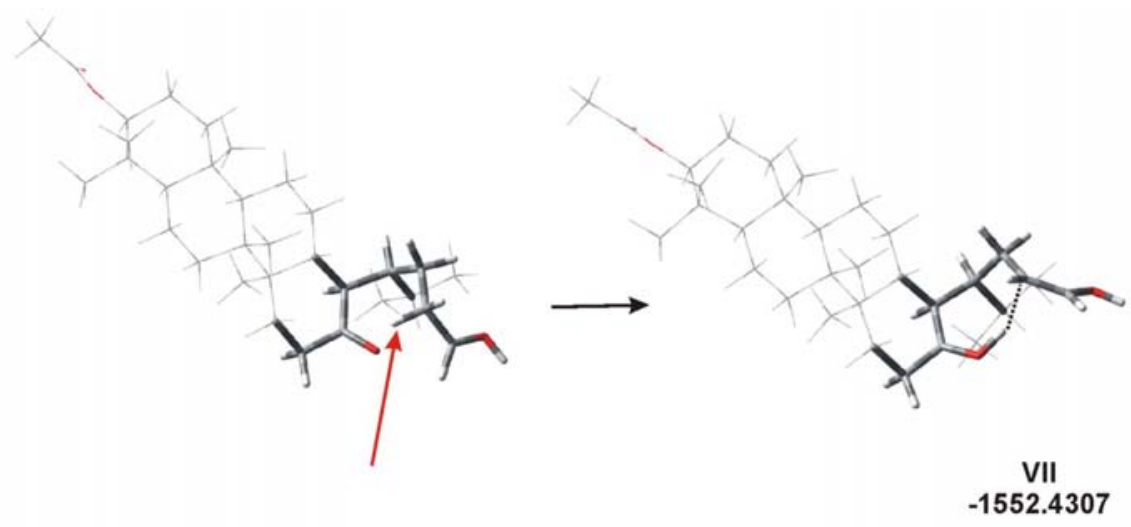

Figure 6S. The formation of enol during structure optimization. The $\mathrm{H}$ atom from $\mathrm{CH}_{2}$ group (red arrow) moved to $\mathrm{C}=\mathrm{O}$

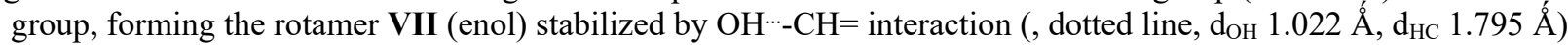

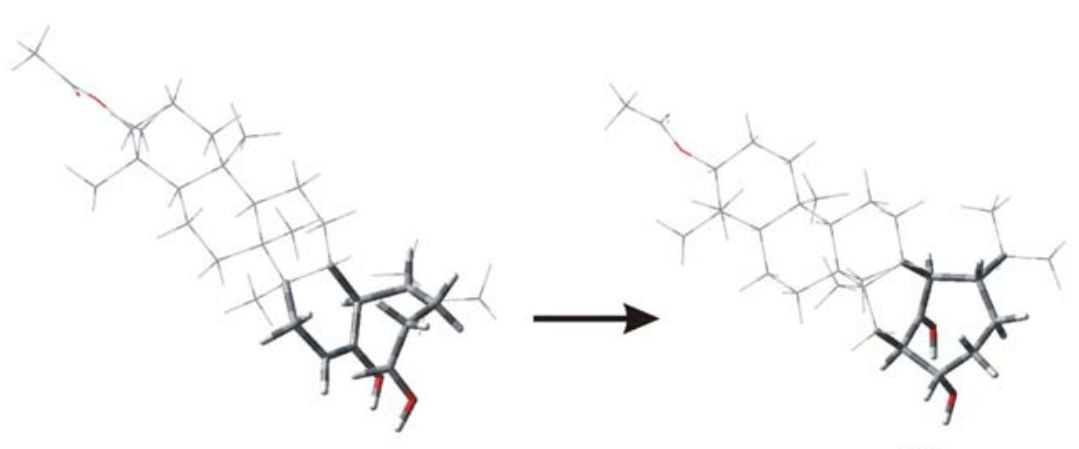

VIII
-1552.4407
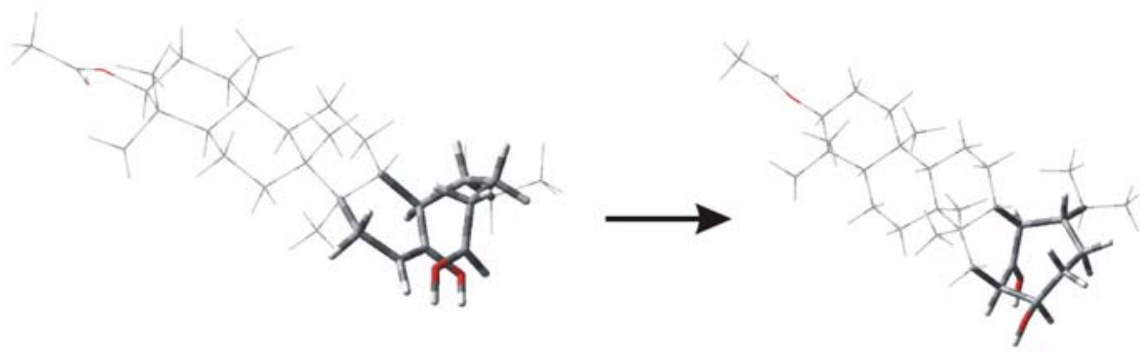

IX

$-1552.4319$

Figure 7S. The transformation of $\mathbf{1 0}$ (enole form) to cyclic product 22. Depending on the orientation of $\left.\mathrm{CH}^{(}=\mathrm{OH}^{+}\right)$ group, two configurations of $\mathrm{CH}(\mathrm{OH})$ center were obtained. 


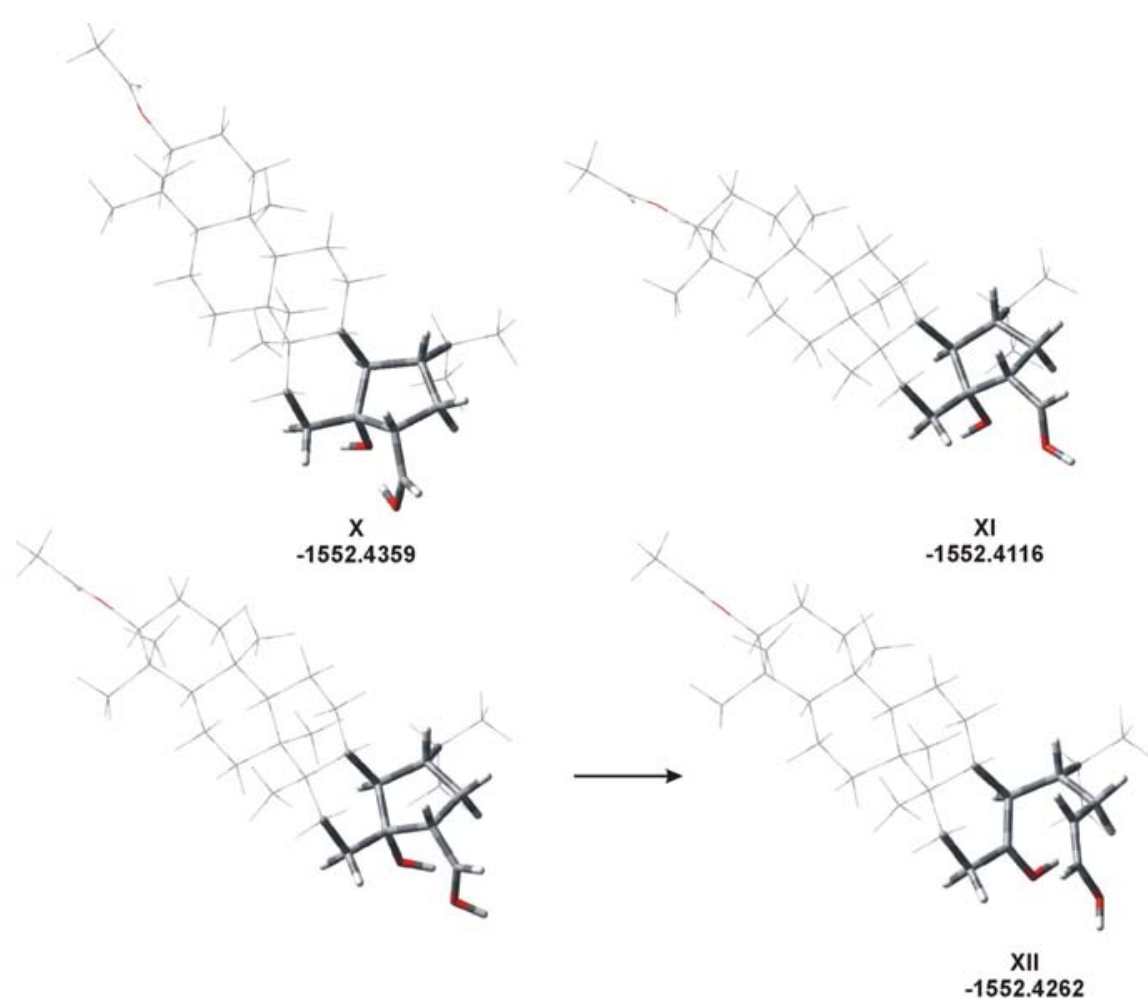

Figure 8S. Two structures, 11 with and without hydrogen bonds (on the top). Below, the structure with opposite hydrogen bond patterns that in X. During optimization, the isomer XII with the open chain fragment was obtained.

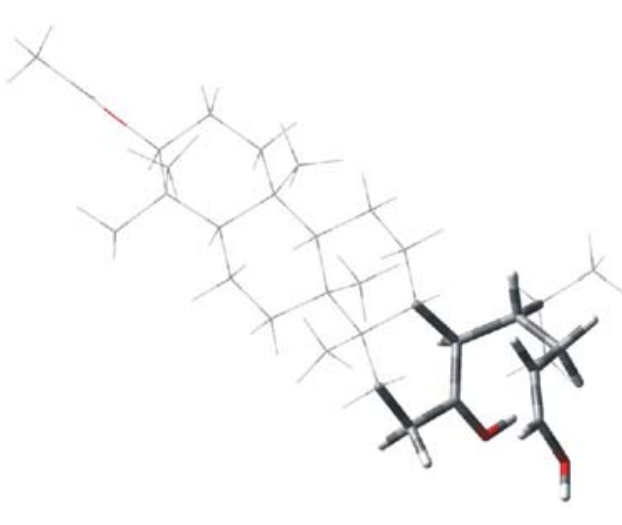

XII

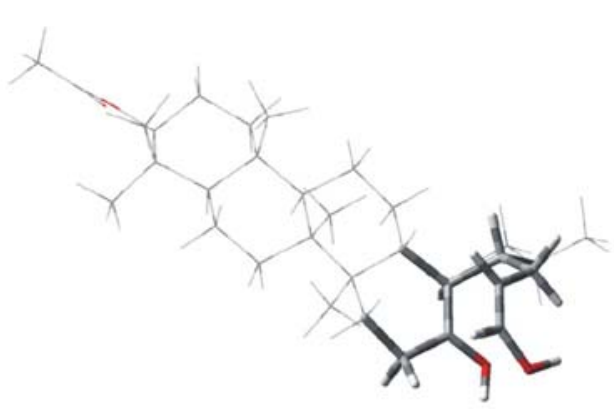

XIII

Figure 9S. Two arrangements of hydrogen bond pattern obtained during various optimizations. In both structures, $\mathrm{O}-\mathrm{H}$ bonds are parallel.

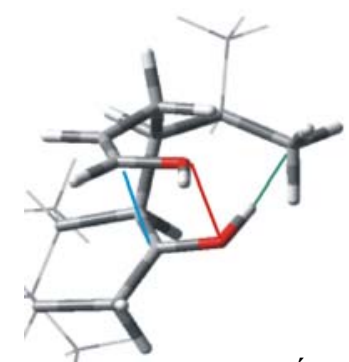

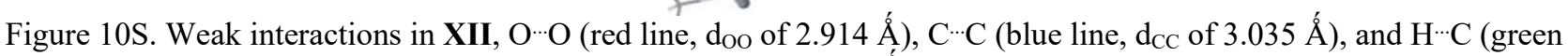
line, $\mathrm{d}_{\mathrm{HC}}$ of $2.275 \AA$ ). 


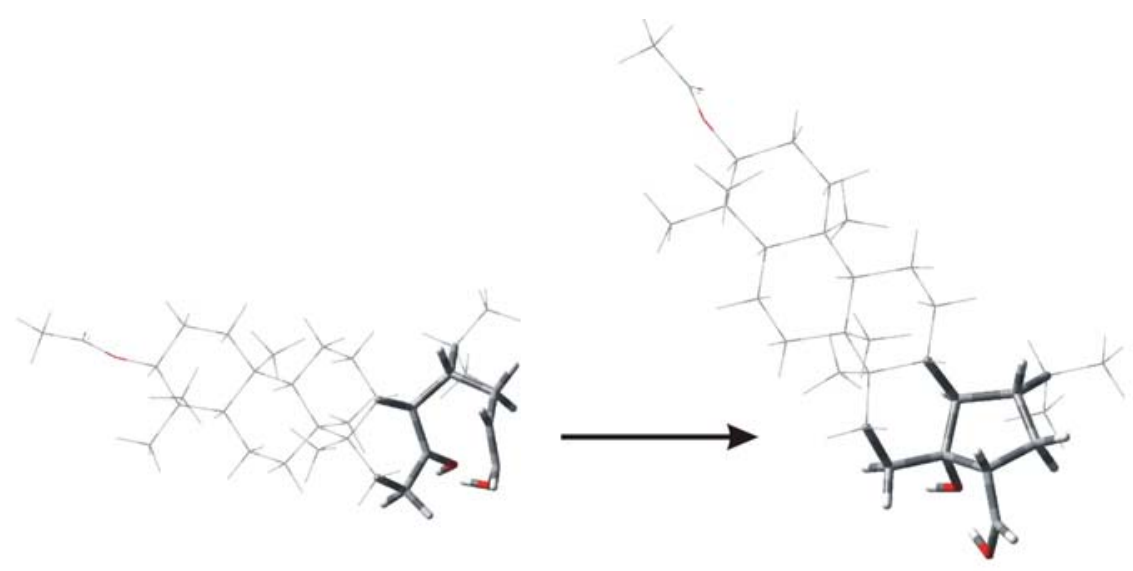

Figure 11S. Formation of $\mathbf{1 1}$ from a linear rotamer (cf. Fig. 8S).

Atomic coordinates of the key structures. Structures have been optimized at the B3LYP/6-31G(2d,p) theory level; the molecular energies have been estimated at the B3LYP/6-311++G(2d,p) theory level.

$\begin{array}{lrrr}\text { I (Fig.2S), E }=\mathbf{- 1 5 5 2 . 0 8 0 2} & & \\ \text { O 1 } & & & \\ \mathrm{C} & 0.00000000 & 0.00000000 & 0.00000000 \\ \mathrm{C} & 1.54654447 & 0.00000000 & 0.00000000 \\ \mathrm{C} & 1.99058940 & 1.50516543 & 0.00000000 \\ \mathrm{C} & 1.39502281 & 2.43392187 & 1.11498412 \\ \mathrm{C} & -0.15025455 & 2.28314783 & 1.06202134 \\ \mathrm{C} & -0.62869066 & 0.82761398 & 1.11278400 \\ \mathrm{H} & -0.35210133 & 0.38447222 & -0.96288258 \\ \mathrm{H} & -0.61618760 & 2.82967194 & 1.88798071 \\ \mathrm{H} & -0.52468966 & 2.74209045 & 0.13689034 \\ \mathrm{H} & -0.40457998 & 0.36889792 & 2.08175519 \\ \mathrm{H} & -1.71620847 & 0.79821156 & 0.99638087 \\ \mathrm{H} & 1.55426156 & 1.90017294 & -0.93243628 \\ \mathrm{C} & 1.85305782 & 2.08296457 & 2.55403338 \\ \mathrm{H} & 2.86349734 & 1.67528911 & 2.60355580 \\ \mathrm{H} & 1.82172152 & 2.96177419 & 3.20229725 \\ \mathrm{H} & 1.19497337 & 1.34040443 & 3.00942152 \\ \mathrm{C} & 1.75134374 & 3.91931377 & 0.69866210 \\ \mathrm{H} & 1.23943541 & 4.01951442 & -0.26569905 \\ \mathrm{C} & 3.50454035 & 1.72028297 & -0.13788201 \\ \mathrm{H} & 3.91688797 & 1.04823006 & -0.89564457 \\ \mathrm{H} & 4.02409541 & 1.47149434 & 0.79373830 \\ \mathrm{C} & 3.81105288 & 3.16078265 & -0.56735862 \\ \mathrm{H} & 3.37722366 & 3.29808064 & -1.56285848 \\ \mathrm{H} & 4.89406427 & 3.27978519 & -0.68193742 \\ \mathrm{C} & 3.26253240 & 4.24025023 & 0.40138667 \\ \mathrm{C} & 2.00179190 & -0.66627397 & -1.31914670 \\ \mathrm{H} & 3.07900935 & -0.85076014 & -1.33511119 \\ \mathrm{H} & 1.50583653 & -1.63356057 & -1.43629862 \\ \mathrm{H} & 1.74979899 & -0.04984762 & -2.18931925 \\ \mathrm{C} & 2.11825542 & -0.83549927 & 1.16314140 \\ \mathrm{H} & 1.72625144 & -0.54424447 & 2.13769107 \\ \mathrm{H} & 1.87568337 & -1.89072952 & 1.01586126 \\ \mathrm{H} & 3.20809981 & -0.74750114 & 1.20152212 \\ \mathrm{O} & -0.45120108 & -1.37648176 & 0.10220555 \\ \mathrm{C} & -1.62917408 & -1.68451111 & -0.48181208 \\ \mathrm{O} & -2.32135398 & -0.89234606 & -1.07356085 \\ \mathrm{C} & 4.13114906 & 4.18708417 & 1.68282972 \\ \mathrm{H} & 5.18250720 & 4.37126285 & 1.45188531 \\ \mathrm{H} & 3.83123217 & 4.90670742 & 2.44657078 \\ \mathrm{H} & 3.36169858 & 5.70106757 & -0.27646009\end{array}$




\begin{tabular}{|c|c|c|c|}
\hline $\mathrm{C}$ & 1.13295382 & 4.99662681 & 1.60458692 \\
\hline $\mathrm{H}$ & 0.07141248 & 4.79259815 & 1.76919760 \\
\hline $\mathrm{H}$ & 1.59923662 & 4.98915052 & 2.59671405 \\
\hline $\mathrm{C}$ & 2.72530201 & 6.76614850 & 0.69005544 \\
\hline $\mathrm{C}$ & 1.26699888 & 6.40293079 & 1.00537005 \\
\hline $\mathrm{H}$ & 0.83658014 & 7.11875359 & 1.71019605 \\
\hline $\mathrm{H}$ & 0.66071457 & 6.47807146 & 0.09270902 \\
\hline $\mathrm{C}$ & 2.65528245 & 5.71873964 & -1.65938545 \\
\hline $\mathrm{H}$ & 2.48801405 & 6.73682281 & -2.01593801 \\
\hline $\mathrm{H}$ & 3.26684436 & 5.22250644 & -2.41746595 \\
\hline $\mathrm{H}$ & 1.67985580 & 5.23160480 & -1.65811795 \\
\hline $\mathrm{H}$ & 3.28444822 & 6.70253003 & 1.63095764 \\
\hline $\mathrm{C}$ & 2.88473264 & 8.24722115 & 0.18778604 \\
\hline $\mathrm{H}$ & 2.32740681 & 8.30770553 & -0.75447139 \\
\hline $\mathrm{C}$ & 4.84101150 & 6.09597368 & -0.51231586 \\
\hline $\mathrm{H}$ & 5.39436471 & 6.06737172 & 0.43042127 \\
\hline $\mathrm{H}$ & 5.32538230 & 5.37949500 & -1.18264840 \\
\hline $\mathrm{C}$ & 5.00134349 & 7.51287884 & -1.09140683 \\
\hline $\mathrm{H}$ & 4.54226675 & 7.57638136 & -2.08520400 \\
\hline $\mathrm{C}$ & 4.34418796 & 8.52725143 & -0.17790748 \\
\hline $\mathrm{H}$ & 6.05516924 & 7.78236568 & -1.19463765 \\
\hline $\mathrm{C}$ & 2.12897018 & 9.22730483 & 3.73673675 \\
\hline $\mathrm{H}$ & 1.37062115 & 10.01242008 & 3.79243976 \\
\hline $\mathrm{C}$ & 2.29619570 & 9.32708575 & 1.14168766 \\
\hline $\mathrm{H}$ & 1.26113749 & 9.02392615 & 1.33996172 \\
\hline $\mathrm{C}$ & 3.04508825 & 9.38222713 & 2.50680824 \\
\hline $\mathrm{H}$ & 3.81275854 & 8.60329092 & 2.55122322 \\
\hline $\mathrm{H}$ & 3.59755922 & 10.32311656 & 2.58205418 \\
\hline $\mathrm{O}$ & 4.96081316 & 9.48173990 & 0.24927394 \\
\hline $\mathrm{C}$ & 2.91783989 & 9.23243188 & 5.02440788 \\
\hline $\mathrm{O}$ & 2.72090599 & 9.97244387 & 5.95558061 \\
\hline $\mathrm{H}$ & 3.73909702 & 8.47701794 & 5.06388118 \\
\hline $\mathrm{C}$ & 2.18307049 & 10.73960948 & 0.49141339 \\
\hline $\mathrm{C}$ & 1.41043105 & 11.70517454 & 1.40561114 \\
\hline $\mathrm{H}$ & 1.35295187 & 12.69889791 & 0.94967965 \\
\hline $\mathrm{H}$ & 0.38193079 & 11.35897507 & 1.56936498 \\
\hline $\mathrm{H}$ & 1.88291086 & 11.82625158 & 2.38383334 \\
\hline $\mathrm{C}$ & -1.94844104 & -3.14788343 & -0.29241256 \\
\hline $\mathrm{H}$ & -2.91102198 & -3.37124281 & -0.75064954 \\
\hline $\mathrm{H}$ & -1.16720496 & -3.76397609 & -0.74703780 \\
\hline $\mathrm{H}$ & -1.97455181 & -3.39144537 & 0.77342339 \\
\hline $\mathrm{C}$ & 1.51126744 & 10.71817155 & -0.89088726 \\
\hline $\mathrm{H}$ & 2.11870646 & 10.21767454 & -1.65003349 \\
\hline $\mathrm{H}$ & 0.53420848 & 10.21908831 & -0.85542840 \\
\hline $\mathrm{H}$ & 1.34086849 & 11.74051992 & -1.24358552 \\
\hline $\mathrm{H}$ & 3.19868125 & 11.12803443 & 0.36912078 \\
\hline $\mathrm{H}$ & 1.60757900 & 8.25990061 & 3.681365 \\
\hline
\end{tabular}

II (Fig. 2S) E = -1552.0797

01

$\begin{array}{ll}\text { C } & 0.00000000\end{array}$

C $\quad 1.54642065$

C $\quad 1.98870736$

C $\quad 1.39188644$

C $\quad-0.15346435$

$\mathrm{C} \quad-0.62875235$

$\mathrm{H} \quad-0.35188190$

$\mathrm{H} \quad-0.61786721$

$\mathrm{H} \quad-0.53155579$

$\mathrm{H} \quad-0.40023424$

$\mathrm{H} \quad-1.71644211$

$\mathrm{H} \quad 1.55303552$

C
$0.00000000 \quad 0.00000000$ $\begin{array}{ll}0.00000000 & 0.00000000\end{array}$ $\begin{array}{ll}1.50594377 & 0.00000000\end{array}$ $\begin{array}{ll}2.43467947 & 1.11389923\end{array}$ $2.28060522 \quad 1.06534277$ $\begin{array}{lll}0.82400090 & 1.11531007\end{array}$ $\begin{array}{ll}0.38724750 & -0.96189018\end{array}$ $\begin{array}{ll}2.82561170 & 1.89279618\end{array}$ $\begin{array}{ll}2.73991524 & 0.14199669\end{array}$ $\begin{array}{ll}0.36405710 & 2.08263063\end{array}$ $\begin{array}{ll}0.79139345 & 1.00133600\end{array}$ $\begin{array}{ll}1.90017875 & -0.93303006\end{array}$ $\begin{array}{ll}2.08932406 & 2.55262698\end{array}$ 


\begin{tabular}{|c|c|c|c|}
\hline $\mathrm{H}$ & 2.86971255 & 1.69678449 & 2.60382013 \\
\hline $\mathrm{H}$ & 1.80699744 & 2.96841106 & 3.19936519 \\
\hline $\mathrm{H}$ & 1.20484891 & 1.33812996 & 3.00765757 \\
\hline $\mathrm{C}$ & 1.74203427 & 3.92011684 & 0.69366212 \\
\hline $\mathrm{H}$ & 1.22132256 & 4.01832867 & -0.26588545 \\
\hline $\mathrm{C}$ & 3.50260751 & 1.72272421 & -0.13498600 \\
\hline $\mathrm{H}$ & 3.91951803 & 1.04671778 & -0.88695037 \\
\hline $\mathrm{H}$ & 4.01789730 & 1.48116349 & 0.80061453 \\
\hline $\mathrm{C}$ & 3.81008599 & 3.16155655 & -0.57073320 \\
\hline $\mathrm{H}$ & 3.38779810 & 3.28961420 & -1.57222434 \\
\hline $\mathrm{H}$ & 4.89418454 & 3.28208760 & -0.67492487 \\
\hline $\mathrm{C}$ & 3.25001613 & 4.24696863 & 0.38444351 \\
\hline $\mathrm{C}$ & 2.00155035 & -0.66743128 & -1.31823535 \\
\hline $\mathrm{H}$ & 3.07864327 & -0.85246135 & -1.33308949 \\
\hline $\mathrm{H}$ & 1.50494330 & -1.63457294 & -1.43457933 \\
\hline $\mathrm{H}$ & 1.75040167 & -0.05185702 & -2.18925019 \\
\hline $\mathrm{C}$ & 2.11900196 & -0.83522869 & 1.16325230 \\
\hline $\mathrm{H}$ & 1.73371466 & -0.53939244 & 2.13922151 \\
\hline $\mathrm{H}$ & 1.87118370 & -1.88988728 & 1.01966931 \\
\hline $\mathrm{H}$ & 3.20938803 & -0.75146138 & 1.19570115 \\
\hline $\mathrm{O}$ & -0.45323006 & -1.37697358 & 0.09787480 \\
\hline $\mathrm{C}$ & -1.63194528 & -1.68109534 & -0.48530616 \\
\hline $\mathrm{O}$ & -2.32243505 & -0.88772965 & -1.07764254 \\
\hline $\mathrm{C}$ & 4.11860128 & 4.21405090 & 1.66733522 \\
\hline $\mathrm{H}$ & 5.16544767 & 4.42841473 & 1.44207749 \\
\hline $\mathrm{H}$ & 3.79630323 & 4.91997151 & 2.43406881 \\
\hline $\mathrm{H}$ & 4.10440453 & 3.22645044 & 2.12402269 \\
\hline $\mathrm{C}$ & 3.33017455 & 5.70078871 & -0.31052916 \\
\hline $\mathrm{C}$ & 1.13497566 & 4.99537541 & 1.60895781 \\
\hline $\mathrm{H}$ & 0.07508164 & 4.79334329 & 1.78567271 \\
\hline $\mathrm{H}$ & 1.61039873 & 4.98080605 & 2.59614276 \\
\hline $\mathrm{C}$ & 2.70639130 & 6.77224891 & 0.65950649 \\
\hline $\mathrm{C}$ & 1.25882908 & 6.41082879 & 1.02773602 \\
\hline $\mathrm{H}$ & 0.86800169 & 7.11039878 & 1.76937495 \\
\hline $\mathrm{H}$ & 0.61714012 & 6.50561703 & 0.14087671 \\
\hline $\mathrm{C}$ & 2.59700049 & 5.70503122 & -1.67850107 \\
\hline $\mathrm{H}$ & 2.49148311 & 6.71611985 & -2.07555274 \\
\hline $\mathrm{H}$ & 3.15624119 & 5.13902523 & -2.42758012 \\
\hline $\mathrm{H}$ & 1.59086523 & 5.28812153 & -1.63568507 \\
\hline $\mathrm{H}$ & 3.29457768 & 6.73020422 & 1.58380751 \\
\hline $\mathrm{C}$ & 2.85189116 & 8.25106044 & 0.12353489 \\
\hline $\mathrm{H}$ & 2.37749851 & 8.26229074 & -0.86370846 \\
\hline $\mathrm{C}$ & 4.80675917 & 6.08903075 & -0.58500866 \\
\hline $\mathrm{H}$ & 5.39451494 & 6.01631908 & 0.33362129 \\
\hline $\mathrm{H}$ & 5.25681147 & 5.39479038 & -1.30143945 \\
\hline $\mathrm{C}$ & 4.97418052 & 7.52871122 & -1.10851597 \\
\hline $\mathrm{H}$ & 4.50093555 & 7.64079416 & -2.09071614 \\
\hline $\mathrm{C}$ & 4.33984406 & 8.49044645 & -0.12309651 \\
\hline $\mathrm{H}$ & 6.03146915 & 7.78548219 & -1.20810767 \\
\hline $\mathrm{C}$ & 1.90714247 & 10.36330664 & 3.35646769 \\
\hline $\mathrm{H}$ & 2.47362468 & 10.51664934 & 4.28887991 \\
\hline $\mathrm{C}$ & 2.70505939 & 9.47577359 & 2.39576272 \\
\hline $\mathrm{H}$ & 2.76768520 & 8.47965258 & 2.84696880 \\
\hline $\mathrm{H}$ & 3.72740160 & 9.84966321 & 2.33401245 \\
\hline $\mathrm{O}$ & 5.00753680 & 9.32119752 & 0.45869218 \\
\hline $\mathrm{C}$ & 0.56139795 & 9.83700092 & 3.78717839 \\
\hline $\mathrm{O}$ & 0.09618677 & 8.76220312 & 3.48980159 \\
\hline $\mathrm{H}$ & -0.00928367 & 10.52562104 & 4.45216873 \\
\hline $\mathrm{C}$ & 1.88793761 & 10.69975930 & 0.24672930 \\
\hline $\mathrm{C}$ & 1.18067357 & 10.52825566 & -1.10772972 \\
\hline $\mathrm{H}$ & 0.82088582 & 11.49541583 & -1.47399840 \\
\hline $\mathrm{H}$ & 1.85279435 & 10.12882173 & -1.87552371 \\
\hline $\mathrm{H}$ & 0.31707871 & 9.85795545 & -1.03388817 \\
\hline
\end{tabular}




$\begin{array}{lccc}\mathrm{C} & -1.95690552 & -3.14335838 & -0.29467040 \\ \mathrm{H} & -2.92064854 & -3.36295164 & -0.75236352 \\ \mathrm{H} & -1.17858717 & -3.76316658 & -0.74924815 \\ \mathrm{H} & -1.98366736 & -3.38587159 & 0.77138091 \\ \mathrm{C} & 3.09708408 & 11.63541451 & 0.09820868 \\ \mathrm{H} & 3.61551541 & 11.79856818 & 1.04641767 \\ \mathrm{H} & 3.83568400 & 11.24781298 & -0.60620749 \\ \mathrm{H} & 2.76096217 & 12.61083725 & -0.27211961 \\ \mathrm{H} & 1.16682554 & 11.22737810 & 0.88660779 \\ \mathrm{H} & 1.75393185 & 11.38017840 & 2.97038496 \\ \mathrm{C} & 2.11888213 & 9.33319634 & 0.97109473 \\ \mathrm{H} & 1.10290151 & 8.94696190 & 1.09243870\end{array}$

\section{III (Fig. 2S) $E=\mathbf{- 1 5 5 2 . 0 8 1 0}$}

01

C

C

C

C

$\mathrm{H}$

$\mathrm{H}$

$\mathrm{H}$

$\mathrm{H}$

$\mathrm{H}$

$\mathrm{H}$

C

$\mathrm{H}$

$\mathrm{H}$

$\mathrm{H}$

C

$\mathrm{H}$

C

$\mathrm{H}$

$\mathrm{H}$

C

$\mathrm{H}$

$\mathrm{H}$

C

C

$\mathrm{H}$

$\mathrm{H}$

$\mathrm{H}$

$\mathrm{C}$

$\mathrm{H}$

$\mathrm{H}$

$\mathrm{H}$

$\mathrm{O}$

C

$\mathrm{O}$

$\mathrm{C}$

$\mathrm{H}$

$\mathrm{H}$

$\mathrm{H}$

C

$\mathrm{C}$

$\mathrm{H}$

$\mathrm{H}$

C

C

$\mathrm{H}$

$\mathrm{H}$ $\begin{array}{lll}0.00000000 & 0.00000000 & 0.00000000\end{array}$ $\begin{array}{lll}1.54618155 & 0.00000000 & 0.00000000\end{array}$ $\begin{array}{lll}1.98617191 & 1.50684957 & 0.00000000\end{array}$ $\begin{array}{lll}1.39178675 & 2.43009266 & 1.11941777\end{array}$ $\begin{array}{lll}-0.15334580 & 2.27395929 & 1.07830716\end{array}$ $\begin{array}{lll}-0.62862958 & 0.81719330 & 1.12040243\end{array}$ $\begin{array}{lll}-0.35161712 & 0.39348112 & -0.95909237\end{array}$ $\begin{array}{lll}-0.61256171 & 2.81299558 & 1.91247237\end{array}$ $\begin{array}{lll}-0.53632091 & 2.73932759 & 0.15986217\end{array}$ $\begin{array}{lll}-0.40187101 & 0.35188389 & 2.08565518\end{array}$ $\begin{array}{lll}-1.71642684 & 0.78552071 & 1.00504497\end{array}$ $\begin{array}{lll}1.54487386 & 1.90092765 & -0.93054730\end{array}$ $\begin{array}{lll}1.86369542 & 2.08674005 & 2.55539538\end{array}$ $\begin{array}{lll}2.87890411 & 1.68995240 & 2.59708392\end{array}$ $\begin{array}{lll}1.83062965 & 2.96947379 & 3.19799203\end{array}$ $\begin{array}{lll}1.21679524 & 1.33977794 & 3.02022657\end{array}$ $\begin{array}{lll}1.73690299 & 3.91632434 & 0.70015848\end{array}$ $\begin{array}{lll}1.22167597 & 4.00863586 & -0.26323141\end{array}$ $\begin{array}{llll}3.49883928 & 1.72908629 & -0.14097857\end{array}$ $\begin{array}{llll}3.91323457 & 1.05914924 & -0.89963450\end{array}$ $\begin{array}{lll}4.01876597 & 1.48205976 & 0.79037189\end{array}$ $\begin{array}{llll}3.80372854 & 3.17248904 & -0.56651303\end{array}$ $3.37815298 \quad 3.31110948-1.56580433$ $\begin{array}{llll}4.88776770 & 3.29316370 & -0.67190677\end{array}$ $\begin{array}{llll}3.24578925 & 4.24858341 & 0.39973583\end{array}$ $\begin{array}{llll}2.00237483 & -0.66647211 & -1.31836629\end{array}$ $\begin{array}{llll}3.08039513 & -0.84596886 & -1.33412140\end{array}$ $\begin{array}{llll}1.51023337 & -1.63599390 & -1.43405205\end{array}$ $\begin{array}{lll}1.74733525 & -0.05141148 & -2.18861588\end{array}$ $\begin{array}{lll}2.11768878 & -0.83473449 & 1.16418586\end{array}$ $\begin{array}{lll}1.73318038 & -0.53503259 & 2.13906279\end{array}$ $\begin{array}{lll}1.86762315 & -1.88942452 & 1.02366332\end{array}$ $\begin{array}{lll}3.20827833 & -0.75381752 & 1.19674331\end{array}$ $\begin{array}{lll}-0.45541038 & -1.37709142 & 0.08957768\end{array}$ $\begin{array}{llll}-1.62618870 & -1.67947910 & -0.50995127\end{array}$ $\begin{array}{lll}-2.30790592 & -0.88557458 & -1.11187204\end{array}$ $\begin{array}{llll}4.11298737 & 4.21499019 & 1.68321218\end{array}$ $\begin{array}{llll}5.15551390 & 4.44861186 & 1.45502752\end{array}$ $\begin{array}{lll}3.77917873 & 4.91017829 & 2.45497499\end{array}$ $\begin{array}{lll}4.11706483 & 3.22334601 & 2.13170064\end{array}$ $\begin{array}{lll}3.32656730 & 5.70671575 & -0.28247856\end{array}$ $\begin{array}{lll}1.11167411 & 4.98959135 & 1.60569037\end{array}$ $\begin{array}{lll}0.05067021 & 4.77778584 & 1.76619469\end{array}$ $\begin{array}{lll}1.57507903 & 4.99853769 & 2.59743879\end{array}$ $\begin{array}{llll}2.69387097 & 6.76439104 & 0.68991791\end{array}$ $\begin{array}{lll}1.23486187 & 6.39397992 & 1.00210876\end{array}$ $\begin{array}{lll}0.80291895 & 7.10345645 & 1.71104491\end{array}$ $\begin{array}{lll}0.62865253 & 6.46485603 & 0.08827944\end{array}$ 


\begin{tabular}{|c|c|c|c|}
\hline $\mathrm{C}$ & 2.60995482 & 5.71662857 & -1.66152947 \\
\hline $\mathrm{H}$ & 2.38133350 & 6.73199937 & -1.99250877 \\
\hline $\mathrm{H}$ & 3.24109519 & 5.27116724 & -2.43575984 \\
\hline $\mathrm{H}$ & 1.66360799 & 5.17661402 & -1.66578998 \\
\hline $\mathrm{H}$ & 3.24217726 & 6.69781051 & 1.63303952 \\
\hline $\mathrm{C}$ & 2.83988253 & 8.24915512 & 0.19999230 \\
\hline $\mathrm{H}$ & 2.22737800 & 8.32551574 & -0.70741716 \\
\hline $\mathrm{C}$ & 4.79968104 & 6.11732282 & -0.53496022 \\
\hline $\mathrm{H}$ & 5.35696369 & 6.12304752 & 0.40606600 \\
\hline $\mathrm{H}$ & 5.29493400 & 5.39294866 & -1.18933905 \\
\hline $\mathrm{C}$ & 4.92512735 & 7.51837963 & -1.15254046 \\
\hline $\mathrm{H}$ & 4.45152528 & 7.54630585 & -2.14140496 \\
\hline $\mathrm{C}$ & 4.26661665 & 8.55678816 & -0.26821252 \\
\hline $\mathrm{H}$ & 5.97106714 & 7.80706387 & -1.28322791 \\
\hline $\mathrm{C}$ & 2.49793091 & 9.50025726 & 3.80597762 \\
\hline $\mathrm{H}$ & 3.10587227 & 10.01454971 & 4.56803495 \\
\hline $\mathrm{C}$ & 2.31723913 & 9.31752278 & 1.20780893 \\
\hline $\mathrm{H}$ & 1.33525528 & 8.96607532 & 1.54604771 \\
\hline $\mathrm{C}$ & 3.23468960 & 9.44261765 & 2.46120892 \\
\hline $\mathrm{H}$ & 3.92908127 & 8.60021037 & 2.50718531 \\
\hline $\mathrm{H}$ & 3.86217458 & 10.32932428 & 2.35480629 \\
\hline $\mathrm{O}$ & 4.85798058 & 9.57186491 & 0.04140068 \\
\hline $\mathrm{C}$ & 2.15009717 & 8.16822259 & 4.42283337 \\
\hline $\mathrm{O}$ & 2.50173302 & 7.08721339 & 4.01249519 \\
\hline $\mathrm{H}$ & 1.53481417 & 8.23894835 & 5.34873596 \\
\hline $\mathrm{C}$ & 2.05379908 & 10.70269915 & 0.53820720 \\
\hline $\mathrm{C}$ & 1.46143469 & 11.71939567 & 1.52875517 \\
\hline $\mathrm{H}$ & 1.29393509 & 12.67952226 & 1.02967251 \\
\hline $\mathrm{H}$ & 0.49158517 & 11.38173431 & 1.91693296 \\
\hline $\mathrm{H}$ & 2.11961860 & 11.91096313 & 2.38021018 \\
\hline $\mathrm{C}$ & -1.95705678 & -3.14089075 & -0.32250272 \\
\hline $\mathrm{H}$ & -2.90216279 & -3.36435350 & -0.81583838 \\
\hline $\mathrm{H}$ & -1.16083871 & -3.76403201 & -0.73958563 \\
\hline $\mathrm{H}$ & -2.02731732 & -3.37460795 & 0.74368101 \\
\hline $\mathrm{C}$ & 1.12289425 & 10.61297270 & -0.68311155 \\
\hline $\mathrm{H}$ & 1.57308591 & 10.08236200 & -1.52615228 \\
\hline $\mathrm{H}$ & 0.18056163 & 10.10914166 & -0.43262307 \\
\hline $\mathrm{H}$ & 0.87050861 & 11.61677875 & -1.04040316 \\
\hline $\mathrm{H}$ & 3.02085806 & 11.08996884 & 0.20319014 \\
\hline $\mathrm{H}$ & 1.57194171 & 10.08782145 & 3.75957210 \\
\hline \multicolumn{4}{|c|}{ tatomer $\mathrm{E}=-\mathbf{1 5 5 2 . 0 6 5 5}($ Fig.3S), } \\
\hline $\mathrm{C}$ & 0.00000000 & 0.00000000 & 0.00000000 \\
\hline $\mathrm{C}$ & 1.54618058 & 0.00000000 & 0.00000000 \\
\hline $\mathrm{C}$ & 1.98952156 & 1.50500586 & 0.00000000 \\
\hline $\mathrm{C}$ & 1.39469640 & 2.43298496 & 1.11738103 \\
\hline $\mathrm{C}$ & -0.15050579 & 2.27943933 & 1.06930277 \\
\hline $\mathrm{C}$ & -0.62745916 & 0.82312792 & 1.11649158 \\
\hline $\mathrm{H}$ & -0.35362293 & 0.38625386 & -0.96149773 \\
\hline $\mathrm{H}$ & -0.61262100 & 2.82209928 & 1.89960837 \\
\hline $\mathrm{H}$ & -0.52821550 & 2.74273528 & 0.14755365 \\
\hline $\mathrm{H}$ & -0.39773872 & 0.36203677 & 2.08304477 \\
\hline $\mathrm{H}$ & -1.71540083 & 0.79112883 & 1.00356609 \\
\hline $\mathrm{H}$ & 1.55297752 & 1.90052448 & -0.93199432 \\
\hline $\mathrm{C}$ & 1.86010783 & 2.08630979 & 2.55507282 \\
\hline $\mathrm{H}$ & 2.86802039 & 1.67196960 & 2.59824109 \\
\hline $\mathrm{H}$ & 1.84257212 & 2.97081030 & 3.19607350 \\
\hline $\mathrm{H}$ & 1.20023905 & 1.35194943 & 3.02227174 \\
\hline $\mathrm{C}$ & 1.74985842 & 3.91623707 & 0.69740348 \\
\hline $\mathrm{H}$ & 1.25378308 & 4.00736770 & -0.27616289 \\
\hline $\mathrm{C}$ & 3.50418425 & 1.71845953 & -0.14042140 \\
\hline $\mathrm{H}$ & 3.91324124 & 1.05118419 & -0.90449770 \\
\hline
\end{tabular}




\begin{tabular}{|c|c|c|c|}
\hline $\mathrm{H}$ & 4.02384298 & 1.46023182 & 0.78857134 \\
\hline $\mathrm{C}$ & 3.81616406 & 3.16216863 & -0.55595709 \\
\hline $\mathrm{H}$ & 3.38937774 & 3.31352442 & -1.55309410 \\
\hline $\mathrm{H}$ & 4.90073492 & 3.27727545 & -0.66326620 \\
\hline $\mathrm{C}$ & 3.26602021 & 4.23406837 & 0.41837077 \\
\hline $\mathrm{C}$ & 2.00195849 & -0.66979469 & -1.31663123 \\
\hline $\mathrm{H}$ & 3.07934187 & -0.85276101 & -1.33098895 \\
\hline $\mathrm{H}$ & 1.50688123 & -1.63797230 & -1.43091635 \\
\hline $\mathrm{H}$ & 1.74990122 & -0.05639376 & -2.18885281 \\
\hline $\mathrm{C}$ & 2.11512467 & -0.83411033 & 1.16571465 \\
\hline $\mathrm{H}$ & 1.72128244 & -0.53995995 & 2.13884626 \\
\hline $\mathrm{H}$ & 1.87269289 & -1.88989550 & 1.02063336 \\
\hline $\mathrm{H}$ & 3.20491520 & -0.74633534 & 1.20645326 \\
\hline $\mathrm{O}$ & -0.45177659 & -1.37801214 & 0.10007758 \\
\hline $\mathrm{C}$ & -1.62681480 & -1.68785664 & -0.48604628 \\
\hline $\mathrm{O}$ & -2.31857605 & -0.89979454 & -1.08414552 \\
\hline $\mathrm{C}$ & 4.12977759 & 4.17634072 & 1.70295050 \\
\hline $\mathrm{H}$ & 5.16948938 & 4.42516902 & 1.47868607 \\
\hline $\mathrm{H}$ & 3.79268253 & 4.85558024 & 2.48770113 \\
\hline $\mathrm{H}$ & 4.14089434 & 3.17589602 & 2.13063084 \\
\hline $\mathrm{C}$ & 3.36895780 & 5.69229867 & -0.24348962 \\
\hline $\mathrm{C}$ & 1.10930903 & 5.00289750 & 1.57958153 \\
\hline $\mathrm{H}$ & 0.04467191 & 4.79586275 & 1.72070998 \\
\hline $\mathrm{H}$ & 1.55391378 & 5.01106710 & 2.58180570 \\
\hline $\mathrm{C}$ & 2.73563910 & 6.74763810 & 0.72311774 \\
\hline $\mathrm{C}$ & 1.25500987 & 6.40440149 & 0.96847083 \\
\hline $\mathrm{H}$ & 0.80024511 & 7.12181558 & 1.65600893 \\
\hline $\mathrm{H}$ & 0.68084490 & 6.46971265 & 0.03646102 \\
\hline $\mathrm{C}$ & 2.67082669 & 5.73773049 & -1.63072422 \\
\hline $\mathrm{H}$ & 2.46314246 & 6.77081890 & -1.91776476 \\
\hline $\mathrm{H}$ & 3.30699025 & 5.30523881 & -2.40887657 \\
\hline $\mathrm{H}$ & 1.71958889 & 5.20658706 & -1.66130397 \\
\hline $\mathrm{H}$ & 3.22023773 & 6.62713149 & 1.70030716 \\
\hline $\mathrm{C}$ & 3.02108169 & 8.20521341 & 0.33026051 \\
\hline $\mathrm{C}$ & 4.83918231 & 6.11806871 & -0.46736689 \\
\hline $\mathrm{H}$ & 5.35485799 & 6.19682061 & 0.49340804 \\
\hline $\mathrm{H}$ & 5.37825919 & 5.37478164 & -1.06337890 \\
\hline $\mathrm{C}$ & 4.93081058 & 7.48381847 & -1.15551983 \\
\hline $\mathrm{H}$ & 4.71368278 & 7.40680806 & -2.23047894 \\
\hline $\mathrm{C}$ & 4.02495162 & 8.49373729 & -0.51217775 \\
\hline $\mathrm{H}$ & 5.96685184 & 7.84930037 & -1.08536974 \\
\hline $\mathrm{C}$ & 2.80672734 & 10.32988929 & 3.43035320 \\
\hline $\mathrm{H}$ & 3.44338296 & 11.05434815 & 3.96308607 \\
\hline $\mathrm{C}$ & 2.29728135 & 9.32908922 & 1.08207425 \\
\hline $\mathrm{H}$ & 1.57481486 & 8.85362342 & 1.75117825 \\
\hline $\mathrm{C}$ & 3.29648116 & 10.11082629 & 1.99631712 \\
\hline $\mathrm{H}$ & 4.23702846 & 9.55765787 & 2.04757479 \\
\hline $\mathrm{H}$ & 3.53512151 & 11.07865939 & 1.54656808 \\
\hline $\mathrm{O}$ & 4.31606585 & 9.81309566 & -0.81001314 \\
\hline $\mathrm{C}$ & 2.78321128 & 9.09153390 & 4.29259849 \\
\hline $\mathrm{O}$ & 3.13476743 & 7.98824180 & 3.94828451 \\
\hline $\mathrm{H}$ & 2.41300066 & 9.26168208 & 5.33025467 \\
\hline $\mathrm{C}$ & 1.46964335 & 10.27435204 & 0.15820731 \\
\hline $\mathrm{C}$ & 0.71598746 & 11.35510550 & 0.94855156 \\
\hline $\mathrm{H}$ & 0.14036417 & 11.99267176 & 0.26876318 \\
\hline $\mathrm{H}$ & 0.00332987 & 10.90800269 & 1.65390986 \\
\hline $\mathrm{H}$ & 1.38645193 & 12.00949158 & 1.51325074 \\
\hline $\mathrm{C}$ & -1.94709421 & -3.15081417 & -0.29074390 \\
\hline $\mathrm{H}$ & -2.90515078 & -3.37759146 & -0.75681617 \\
\hline $\mathrm{H}$ & -1.16113139 & -3.76961954 & -0.73333447 \\
\hline $\mathrm{H}$ & -1.98385537 & -3.38738494 & 0.77640363 \\
\hline $\mathrm{C}$ & 0.47207408 & 9.50114019 & -0.71516606 \\
\hline $\mathrm{H}$ & 0.97235757 & 8.75194578 & -1.33335306 \\
\hline
\end{tabular}




$\begin{array}{cccc}\mathrm{H} & -0.27751638 & 8.98555629 & -0.10234615 \\ \mathrm{H} & -0.06513267 & 10.18371675 & -1.38285565 \\ \mathrm{H} & 2.17886827 & 10.77715438 & -0.50714920 \\ \mathrm{H} & 1.80010318 & 10.76896749 & 3.47197103 \\ \mathrm{H} & 5.10312036 & 9.83465932 & -1.36343275\end{array}$

tautomer $\mathrm{E}=\mathbf{- 1 5 5 2 . 0 6 5 3}$ (Fig.3S),

01

$\begin{array}{llll}\text { C } & 0.00000000 & 0.00000000 & 0.00000000\end{array}$

$\begin{array}{llll}\mathrm{C} & 1.54619375 & 0.00000000 & 0.00000000\end{array}$

$\begin{array}{llll}\mathrm{C} & 1.98921574 & 1.50522580 & 0.00000000\end{array}$

$\begin{array}{llll}\mathrm{C} & 1.39336918 & 2.43415134 & 1.11431521\end{array}$

$\begin{array}{llll}\text { C } & -0.15236963 & 2.27943928 & 1.06695612\end{array}$

$\begin{array}{llll}\mathrm{C} & -0.62871465 & 0.82305282 & 1.11599224\end{array}$

$\begin{array}{llll}\mathrm{H} & -0.35289385 & 0.38681369 & -0.96144006\end{array}$

$\begin{array}{llll}\mathrm{H} & -0.61560101 & 2.82425799 & 1.89534053\end{array}$

$\begin{array}{llll}\mathrm{H} & -0.53067930 & 2.73955131 & 0.14401538\end{array}$

$\begin{array}{llll}\mathrm{H} & -0.40068354 & 0.36202591 & 2.08295193\end{array}$

$\begin{array}{llll}\mathrm{H} & -1.71652734 & 0.79062400 & 1.00151818\end{array}$

$\begin{array}{llll}\mathrm{H} & 1.55354186 & 1.89921870 & -0.93318371\end{array}$

$\begin{array}{llll}\text { C } & 1.85607685 & 2.08577036 & 2.55222443\end{array}$

$\begin{array}{lllll}\mathrm{H} & 2.87543746 & 1.69977774 & 2.60093561\end{array}$

$\begin{array}{llll}\mathrm{H} & 1.80551294 & 2.96259196 & 3.20188426\end{array}$

$\begin{array}{llll}\mathrm{H} & 1.21373568 & 1.32784056 & 3.00550611\end{array}$

$\begin{array}{lllll}\mathrm{C} & 1.74230664 & 3.92057505 & 0.69550684\end{array}$

$\begin{array}{llll}\mathrm{H} & 1.20633116 & 4.02825570 & -0.25515606\end{array}$

$\begin{array}{lllll}\text { C } & 3.50377741 & 1.72417807 & -0.13726734\end{array}$

$\begin{array}{llll}\mathrm{H} & 3.92390328 & 1.03967778 & -0.87985867\end{array}$

$\begin{array}{llll}\mathrm{H} & 4.02112858 & 1.50116698 & 0.80199335\end{array}$

$\begin{array}{llll}\mathrm{C} & 3.79449130 & 3.15933321 & -0.59365105\end{array}$

$\mathrm{H} \quad 3.35065464 \quad 3.27366137 \quad-1.58741076$

$\begin{array}{llll}\mathrm{H} & 4.87511155 & 3.29107508 & -0.72063477\end{array}$

$\begin{array}{llll}\mathrm{C} & 3.24540296 & 4.24779505 & 0.36235498\end{array}$

$\begin{array}{llll}\mathrm{C} & 2.00184184 & -0.66819204 & -1.31757690\end{array}$

$\mathrm{H} \quad 3.07864236 \quad-0.85471023 \quad-1.33033980$

$\mathrm{H} \quad \begin{array}{llll}\mathrm{H} & 1.50384002 & -1.63449784 & -1.43514862\end{array}$

$\begin{array}{llll}\mathrm{H} & 1.75372280 & -0.05173843 & -2.18869272\end{array}$

$\begin{array}{llll}\mathrm{C} & 2.11682425 & -0.83522055 & 1.16396449\end{array}$

$\begin{array}{llll}\mathrm{H} & 1.72427329 & -0.54252935 & 2.13794781\end{array}$

$\begin{array}{llll}\mathrm{H} & 1.87514012 & -1.89120112 & 1.01818518\end{array}$

$\begin{array}{llll}\mathrm{H} & 3.20656297 & -0.74629137 & 1.20329297\end{array}$

$\begin{array}{llll}\mathrm{O} & -0.45258581 & -1.37801193 & 0.09829134\end{array}$

C $\quad-1.62770939-1.68547400 \quad-0.48876189$

$\begin{array}{llll}\text { O } & -2.31828669 & -0.89566771 & -1.08606246\end{array}$

$\begin{array}{llll}\mathrm{C} & 4.13112255 & 4.22698174 & 1.63533513\end{array}$

$\begin{array}{llll}\mathrm{H} & 5.18839025 & 4.31314412 & 1.37268360\end{array}$

$\begin{array}{llll}\mathrm{H} & 3.90575280 & 5.02327907 & 2.34555101\end{array}$

$\begin{array}{llll}\mathrm{H} & 4.02796034 & 3.28583853 & 2.17175877\end{array}$

$\begin{array}{llll}\text { C } & 3.31175735 & 5.69322457 & -0.34093999\end{array}$

$\begin{array}{llll}\mathrm{C} & 1.15537044 & 4.99544884 & 1.62780955\end{array}$

$\begin{array}{llll}\mathrm{H} & 0.10623088 & 4.77980830 & 1.85030384\end{array}$

$\begin{array}{llll}\mathrm{H} & 1.67788961 & 5.00825376 & 2.59045152\end{array}$

$\begin{array}{llll}\mathrm{C} & 2.68770074 & 6.76346930 & 0.61412208\end{array}$

$\begin{array}{llll}\mathrm{C} & 1.24707632 & 6.38862175 & 0.99841171\end{array}$

$\begin{array}{llll}\mathrm{H} & 0.83866597 & 7.12343864 & 1.70005690\end{array}$

$\begin{array}{lllll}\mathrm{H} & 0.60188289 & 6.43273734 & 0.11061958\end{array}$

$\begin{array}{lllll}\text { C } & 2.58179879 & 5.70659607 & -1.71017409\end{array}$

$\mathrm{H} \quad 2.56004958 \quad 6.71838994 \quad-2.11917096$

$\begin{array}{lllll}\mathrm{H} & 3.10258524 & 5.08899965 & -2.44616947\end{array}$

$\mathrm{H} \quad \begin{array}{llll}\mathrm{H} & 1.55001894 & 5.35606421 & -1.65970420\end{array}$

$\begin{array}{llll}\mathrm{H} & 3.27808977 & 6.75140870 & 1.53439585\end{array}$

$\begin{array}{llll}\mathrm{C} & 2.75308016 & 8.22827481 & 0.08830405\end{array}$

$\mathrm{H} \quad \begin{array}{llll}\mathrm{H} & 1.90286493 & 8.38643705 & -0.58748604\end{array}$ 


$\begin{array}{rcc}4.78127107 & 6.11243759 & -0.61584532 \\ 5.40320110 & 5.94786816 & 0.27070807 \\ 5.20657140 & 5.47423423 & -1.39964298 \\ 4.89863033 & 7.55159548 & -1.04100766 \\ 3.99278533 & 8.48119959 & -0.73263150 \\ 5.76376640 & 7.83295296 & -1.64154232 \\ 3.95632460 & 9.73320369 & 3.45753286 \\ 4.90541812 & 10.16914797 & 3.80909405 \\ 2.60625012 & 9.25900170 & 1.27329621 \\ 1.97990904 & 8.76888076 & 2.02794196 \\ 3.98165844 & 9.53138973 & 1.93871429 \\ 4.64889671 & 8.68883396 & 1.73470975 \\ 4.45005219 & 10.40020280 & 1.46709406 \\ 4.08397985 & 9.78586296 & -1.16026598 \\ 3.76354103 & 8.48402025 & 4.28086856 \\ 3.69149044 & 7.35675600 & 3.85248526 \\ 3.70286372 & 8.66707181 & 5.37842163 \\ 1.83559155 & 10.53992481 & 0.82589310 \\ 1.90411146 & 11.70752968 & 1.82328408 \\ 1.36993663 & 12.57391907 & 1.41846877 \\ 1.42287786 & 11.45394369 & 2.77634267 \\ 2.92844237 & 12.02820083 & 2.03082455 \\ -1.95066311 & -3.14817440 & -0.29573257 \\ -2.90631302 & -3.37359286 & -0.76741127 \\ -1.16283870 & -3.76802324 & -0.73342065 \\ -1.99439357 & -3.38462913 & 0.77120388 \\ 0.35399536 & 10.23268729 & 0.54862816 \\ 0.21305665 & 9.44049703 & -0.19092846 \\ -0.15676741 & 9.92036322 & 1.46809448 \\ -0.16057840 & 11.12391009 & 0.17395694 \\ 2.29541227 & 10.88405695 & -0.10621196 \\ 3.19379774 & 10.45119235 & 3.78421464 \\ 4.88283736 & 9.87615686 & -1.69395694\end{array}$

\begin{tabular}{lrrr}
\multicolumn{5}{l}{ tautomer } & $\mathbf{E}=\mathbf{- 1 5 5 2 . 0 6 6 8}$ & (Fig. 3S), & \\
0 1 & & & \\
$\mathrm{C}$ & 0.00000000 & 0.00000000 & 0.00000000 \\
$\mathrm{C}$ & 1.54657305 & 0.00000000 & 0.00000000 \\
$\mathrm{C}$ & 1.99158584 & 1.50513936 & 0.00000000 \\
$\mathrm{C}$ & 1.39608028 & 2.43340828 & 1.11494806 \\
$\mathrm{C}$ & -0.14901329 & 2.28376396 & 1.05943244 \\
$\mathrm{C}$ & -0.62902925 & 0.82895045 & 1.11135279 \\
$\mathrm{H}$ & -0.35214021 & 0.38270655 & -0.96356091 \\
$\mathrm{H}$ & -0.61577868 & 2.83194409 & 1.88365811 \\
$\mathrm{H}$ & -0.52096245 & 2.74211518 & 0.13292709 \\
$\mathrm{H}$ & -0.40542154 & 0.37101377 & 2.08087704 \\
$\mathrm{H}$ & -1.71654183 & 0.79979069 & 0.99428194 \\
$\mathrm{H}$ & 1.55612705 & 1.89962723 & -0.93315889 \\
$\mathrm{C}$ & 1.85280453 & 2.08130361 & 2.55426396 \\
$\mathrm{H}$ & 2.85965614 & 1.66477616 & 2.60185985 \\
$\mathrm{H}$ & 1.83148967 & 2.96146849 & 3.20070602 \\
$\mathrm{H}$ & 1.18945268 & 1.34520189 & 3.01286876 \\
$\mathrm{C}$ & 1.75345431 & 3.91930124 & 0.70243147 \\
$\mathrm{H}$ & 1.24314247 & 4.02230523 & -0.26285997 \\
$\mathrm{C}$ & 3.50586659 & 1.71958830 & -0.13748294 \\
$\mathrm{H}$ & 3.91727562 & 1.04880000 & -0.89710325 \\
$\mathrm{H}$ & 4.02513774 & 1.46792993 & 0.79362358 \\
$\mathrm{C}$ & 3.81389142 & 3.16143043 & -0.56267555 \\
$\mathrm{H}$ & 3.38024231 & 3.30260630 & -1.55789627 \\
$\mathrm{H}$ & 4.89719783 & 3.28000969 & -0.67723692 \\
$\mathrm{C}$ & 3.26580552 & 4.23916871 & 0.40846450 \\
$\mathrm{C}$ & 2.00133659 & -0.66655055 & -1.31909898 \\
$\mathrm{H}$ & 3.07867001 & -0.85029976 & -1.33549263 \\
& & & \\
& & &
\end{tabular}




\begin{tabular}{|c|c|c|c|}
\hline $\mathrm{H}$ & 1.50593556 & -1.63426350 & -1.43602927 \\
\hline $\mathrm{H}$ & 1.74888157 & -0.05069026 & -2.18960227 \\
\hline $\mathrm{C}$ & 2.11799057 & -0.83616656 & 1.16281817 \\
\hline $\mathrm{H}$ & 1.73198455 & -0.54038805 & 2.13866730 \\
\hline $\mathrm{H}$ & 1.86943912 & -1.89052101 & 1.01888384 \\
\hline $\mathrm{H}$ & 3.20843848 & -0.75378798 & 1.19630676 \\
\hline $\mathrm{O}$ & -0.45144592 & -1.37691312 & 0.10414787 \\
\hline $\mathrm{C}$ & -1.63003810 & -1.68594523 & -0.47668758 \\
\hline $\mathrm{O}$ & -2.32383661 & -0.89587744 & -1.06959613 \\
\hline $\mathrm{C}$ & 4.13080952 & 4.18005016 & 1.69227525 \\
\hline $\mathrm{H}$ & 5.18240009 & 4.36843081 & 1.46537339 \\
\hline $\mathrm{H}$ & 3.82555770 & 4.89164893 & 2.46123828 \\
\hline $\mathrm{H}$ & 4.09210685 & 3.19288168 & 2.14713075 \\
\hline $\mathrm{C}$ & 3.36983281 & 5.70136222 & -0.26460727 \\
\hline $\mathrm{C}$ & 1.13375364 & 4.99539939 & 1.60900165 \\
\hline $\mathrm{H}$ & 0.07125433 & 4.79083845 & 1.76906390 \\
\hline $\mathrm{H}$ & 1.59635735 & 4.99259812 & 2.60254257 \\
\hline $\mathrm{C}$ & 2.73056550 & 6.75997697 & 0.70405851 \\
\hline $\mathrm{C}$ & 1.27034787 & 6.40102245 & 1.01118063 \\
\hline $\mathrm{H}$ & 0.84917230 & 7.11420312 & 1.72260591 \\
\hline $\mathrm{H}$ & 0.66566257 & 6.48110254 & 0.09755923 \\
\hline $\mathrm{C}$ & 2.67136551 & 5.72476685 & -1.65103380 \\
\hline $\mathrm{H}$ & 2.50081522 & 6.74506466 & -2.00056291 \\
\hline $\mathrm{H}$ & 3.28821383 & 5.23631957 & -2.41040735 \\
\hline $\mathrm{H}$ & 1.69820131 & 5.23336103 & -1.65719419 \\
\hline $\mathrm{H}$ & 3.27993732 & 6.69126460 & 1.64794023 \\
\hline $\mathrm{C}$ & 2.89273035 & 8.24598852 & 0.21927501 \\
\hline $\mathrm{H}$ & 2.33936152 & 8.32233412 & -0.72593311 \\
\hline $\mathrm{C}$ & 4.85030283 & 6.09754828 & -0.49055376 \\
\hline $\mathrm{H}$ & 5.39816496 & 6.06583199 & 0.45520473 \\
\hline $\mathrm{H}$ & 5.34008973 & 5.38478969 & -1.16137526 \\
\hline $\mathrm{C}$ & 5.01059058 & 7.51664338 & -1.06245760 \\
\hline $\mathrm{H}$ & 4.55449042 & 7.58351791 & -2.05759789 \\
\hline $\mathrm{C}$ & 4.34979984 & 8.53118042 & -0.15000392 \\
\hline $\mathrm{H}$ & 6.06411202 & 7.78893741 & -1.16267365 \\
\hline $\mathrm{C}$ & 2.27672890 & 8.50183246 & 3.63883741 \\
\hline $\mathrm{H}$ & 1.22788043 & 8.73963292 & 3.82798860 \\
\hline $\mathrm{C}$ & 2.29703349 & 9.31465779 & 1.18089941 \\
\hline $\mathrm{H}$ & 1.24669150 & 9.03463965 & 1.32549999 \\
\hline $\mathrm{C}$ & 2.98259483 & 9.31269341 & 2.58511600 \\
\hline $\mathrm{H}$ & 4.01847223 & 8.97691514 & 2.49336214 \\
\hline $\mathrm{H}$ & 3.05400764 & 10.35085266 & 2.93146573 \\
\hline $\mathrm{O}$ & 4.96366115 & 9.49487296 & 0.26209876 \\
\hline $\mathrm{C}$ & 2.85562771 & 7.54325240 & 4.36355372 \\
\hline $\mathrm{O}$ & 2.27762383 & 6.78354906 & 5.33909740 \\
\hline $\mathrm{H}$ & 3.89860819 & 7.26692144 & 4.23652764 \\
\hline $\mathrm{C}$ & 2.24825917 & 10.75898213 & 0.58934249 \\
\hline $\mathrm{C}$ & 1.23527793 & 11.61412433 & 1.36950329 \\
\hline $\mathrm{H}$ & 1.26396594 & 12.65528840 & 1.03160714 \\
\hline $\mathrm{H}$ & 0.21322023 & 11.24525040 & 1.21508160 \\
\hline $\mathrm{H}$ & 1.42625579 & 11.61492476 & 2.44664652 \\
\hline $\mathrm{C}$ & -1.94936833 & -3.14895029 & -0.28266181 \\
\hline $\mathrm{H}$ & -2.91108830 & -3.37413193 & -0.74185417 \\
\hline $\mathrm{H}$ & -1.16711293 & -3.76666938 & -0.73326366 \\
\hline $\mathrm{H}$ & -1.97768986 & -3.38846849 & 0.78406779 \\
\hline $\mathrm{C}$ & 1.89592922 & 10.81544747 & -0.90450677 \\
\hline $\mathrm{H}$ & 2.67174997 & 10.37204816 & -1.53444907 \\
\hline $\mathrm{H}$ & 0.94858402 & 10.30305361 & -1.11689326 \\
\hline $\mathrm{H}$ & 1.77897561 & 11.85622905 & -1.22459733 \\
\hline $\mathrm{H}$ & 3.24289938 & 11.19880207 & 0.71054765 \\
\hline $\mathrm{H}$ & 1.35990906 & 7.06380722 & 5.44498310 \\
\hline
\end{tabular}

Tautomer E = -1552.0680 (Fig. 3S) 


\begin{tabular}{|c|c|c|c|}
\hline & & & \\
\hline $\mathrm{C}$ & 0.00000000 & 0.00000000 & 0.00000000 \\
\hline $\mathrm{C}$ & 1.54632788 & 0.00000000 & 0.00000000 \\
\hline $\mathrm{C}$ & 1.98592143 & 1.50681494 & 0.00000000 \\
\hline $\mathrm{C}$ & 1.39207670 & 2.43116199 & 1.11903931 \\
\hline $\mathrm{C}$ & -0.15319918 & 2.27596328 & 1.07656402 \\
\hline $\mathrm{C}$ & -0.62839495 & 0.81906847 & 1.11921726 \\
\hline $\mathrm{H}$ & -0.35171809 & 0.39202526 & -0.95975170 \\
\hline $\mathrm{H}$ & -0.61319909 & 2.81455028 & 1.91067300 \\
\hline $\mathrm{H}$ & -0.53569865 & 2.74143079 & 0.15803607 \\
\hline $\mathrm{H}$ & -0.40156735 & 0.35456475 & 2.08483154 \\
\hline $\mathrm{H}$ & -1.71611009 & 0.78748788 & 1.00398705 \\
\hline $\mathrm{H}$ & 1.54449972 & 1.90082548 & -0.93048473 \\
\hline $\mathrm{C}$ & 1.86181403 & 2.08473454 & 2.55537673 \\
\hline $\mathrm{H}$ & 2.87265451 & 1.67680477 & 2.59769289 \\
\hline $\mathrm{H}$ & 1.83774361 & 2.96721906 & 3.19865416 \\
\hline $\mathrm{H}$ & 1.20713885 & 1.34464705 & 3.01999415 \\
\hline $\mathrm{C}$ & 1.73879799 & 3.91760146 & 0.70082960 \\
\hline $\mathrm{H}$ & 1.22372339 & 4.01247601 & -0.26259315 \\
\hline $\mathrm{C}$ & 3.49867512 & 1.72867902 & -0.14214025 \\
\hline $\mathrm{H}$ & 3.91215427 & 1.05965966 & -0.90203072 \\
\hline $\mathrm{H}$ & 4.01964484 & 1.48074795 & 0.78831449 \\
\hline $\mathrm{C}$ & 3.80208591 & 3.17191369 & -0.56776868 \\
\hline $\mathrm{H}$ & 3.37135049 & 3.31030568 & -1.56461214 \\
\hline $\mathrm{H}$ & 4.88549325 & 3.29284008 & -0.67862986 \\
\hline $\mathrm{C}$ & 3.24839071 & 4.24771154 & 0.40149737 \\
\hline $\mathrm{C}$ & 2.00278812 & -0.66599050 & -1.31860147 \\
\hline $\mathrm{H}$ & 3.08053180 & -0.84730598 & -1.33344270 \\
\hline $\mathrm{H}$ & 1.50936596 & -1.63469734 & -1.43535999 \\
\hline $\mathrm{H}$ & 1.74984329 & -0.05033459 & -2.18907274 \\
\hline $\mathrm{C}$ & 2.11849008 & -0.83487348 & 1.16353689 \\
\hline $\mathrm{H}$ & 1.73446496 & -0.53591368 & 2.13885100 \\
\hline $\mathrm{H}$ & 1.86841695 & -1.88938787 & 1.02244800 \\
\hline $\mathrm{H}$ & 3.20910623 & -0.75402055 & 1.19553475 \\
\hline $\mathrm{O}$ & -0.45481682 & -1.37669759 & 0.09187742 \\
\hline $\mathrm{C}$ & -1.62750807 & -1.67962655 & -0.50432977 \\
\hline $\mathrm{O}$ & -2.31198967 & -0.88495464 & -1.10192721 \\
\hline $\mathrm{C}$ & 4.11808938 & 4.20358752 & 1.68363363 \\
\hline $\mathrm{H}$ & 5.16582980 & 4.40740706 & 1.45010310 \\
\hline $\mathrm{H}$ & 3.80911093 & 4.90886560 & 2.45704085 \\
\hline $\mathrm{H}$ & 4.09954612 & 3.21502723 & 2.13776558 \\
\hline $\mathrm{C}$ & 3.33429260 & 5.70743449 & -0.27884122 \\
\hline $\mathrm{C}$ & 1.11528806 & 4.99126064 & 1.60707942 \\
\hline $\mathrm{H}$ & 0.05630057 & 4.77817717 & 1.77681140 \\
\hline $\mathrm{H}$ & 1.58679194 & 4.99585531 & 2.59621444 \\
\hline $\mathrm{C}$ & 2.68947659 & 6.76678786 & 0.68732010 \\
\hline $\mathrm{C}$ & 1.23299258 & 6.39627801 & 1.00348360 \\
\hline $\mathrm{H}$ & 0.81009300 & 7.10773755 & 1.71479969 \\
\hline $\mathrm{H}$ & 0.62544575 & 6.46412029 & 0.09073730 \\
\hline $\mathrm{C}$ & 2.62901128 & 5.71786119 & -1.66279663 \\
\hline $\mathrm{H}$ & 2.42634841 & 6.73359275 & -2.00830440 \\
\hline $\mathrm{H}$ & 3.25635885 & 5.25010508 & -2.42641009 \\
\hline $\mathrm{H}$ & 1.67099812 & 5.19851825 & -1.66721267 \\
\hline $\mathrm{H}$ & 3.24608732 & 6.70553848 & 1.62841802 \\
\hline $\mathrm{C}$ & 2.83827662 & 8.24808030 & 0.18516251 \\
\hline $\mathrm{H}$ & 2.29663008 & 8.30160108 & -0.76707351 \\
\hline $\mathrm{C}$ & 4.81110350 & 6.11474966 & -0.51887921 \\
\hline $\mathrm{H}$ & 5.36845801 & 6.08759582 & 0.42162190 \\
\hline $\mathrm{H}$ & 5.30056722 & 5.40512486 & -1.19309980 \\
\hline $\mathrm{C}$ & 4.95699178 & 7.53572553 & -1.09302670 \\
\hline $\mathrm{H}$ & 4.48763415 & 7.60091718 & -2.08166271 \\
\hline $\mathrm{C}$ & 4.30020228 & 8.53315251 & -0.16163229 \\
\hline $\mathrm{H}$ & 6.00832581 & 7.81272168 & -1.20259237 \\
\hline
\end{tabular}




$\begin{array}{lrcc}\mathrm{C} & 2.07401550 & 8.66056072 & 3.60840378 \\ \mathrm{H} & 1.07028927 & 9.03568021 & 3.79025654 \\ \mathrm{C} & 2.22014012 & 9.32862743 & 1.11587751 \\ \mathrm{H} & 1.16798134 & 9.04759348 & 1.24290972 \\ \mathrm{C} & 2.87573089 & 9.34971881 & 2.53695688 \\ \mathrm{H} & 3.88839030 & 8.94064647 & 2.46787590 \\ \mathrm{H} & 3.03081548 & 10.39375938 & 2.83267257 \\ \mathrm{O} & 4.92299468 & 9.47015257 & 0.29742564 \\ \mathrm{C} & 2.46763417 & 7.63630186 & 4.36871351 \\ \mathrm{O} & 3.67409799 & 6.99320180 & 4.34028650 \\ \mathrm{H} & 1.82268347 & 7.19105464 & 5.11915385 \\ \mathrm{C} & 2.18933898 & 10.76073493 & 0.49730410 \\ \mathrm{C} & 1.18634414 & 11.64212285 & 1.26056570 \\ \mathrm{H} & 1.22664245 & 12.67613852 & 0.90277133 \\ \mathrm{H} & 0.16051372 & 11.28105820 & 1.11383442 \\ \mathrm{H} & 1.37783464 & 11.66189148 & 2.33753144 \\ \mathrm{C} & -1.95462435 & -3.14220187 & -0.32038967 \\ \mathrm{H} & -2.91137775 & -3.36148587 & -0.79266261 \\ \mathrm{H} & -1.16940446 & -3.76136441 & -0.76374454 \\ \mathrm{H} & -1.99747513 & -3.38586071 & 0.74489167 \\ \mathrm{C} & 1.83879770 & 10.79203725 & -0.99814111 \\ \mathrm{H} & 2.61673451 & 10.34365735 & -1.62210944 \\ \mathrm{H} & 0.89420339 & 10.27213902 & -1.20340851 \\ \mathrm{H} & 1.71768021 & 11.82725167 & -1.33367945 \\ \mathrm{H} & 3.18941418 & 11.19039348 & 0.61225341 \\ \mathrm{H} & 4.26608136 & 7.46749191 & 3.74332927\end{array}$

Protonated compounds calculated as cations (Fig. 4S):

$\begin{array}{lrrr}\mathbf{H}^{+} \text {at } \mathbf{C}=\mathbf{O}, \mathbf{E}=\mathbf{- 1 5 5 2 . 4 3 2 2} & & \\ 1 \mathrm{l} & & & \\ \mathrm{C} & 0.00000000 & 0.00000000 & 0.00000000 \\ \mathrm{C} & 1.54720649 & 0.00000000 & 0.00000000 \\ \mathrm{C} & 1.98377350 & 1.50722698 & 0.00000000 \\ \mathrm{C} & 1.38913102 & 2.42931875 & 1.12111756 \\ \mathrm{C} & -0.15647352 & 2.27224026 & 1.08480863 \\ \mathrm{C} & -0.62583875 & 0.81401746 & 1.12582413 \\ \mathrm{H} & -0.35095345 & 0.40354907 & -0.95589768 \\ \mathrm{H} & -0.61386822 & 2.80680726 & 1.92354951 \\ \mathrm{H} & -0.54538984 & 2.73724041 & 0.16833514 \\ \mathrm{H} & -0.39500580 & 0.34806164 & 2.08941729 \\ \mathrm{H} & -1.71320600 & 0.78188349 & 1.01430165 \\ \mathrm{H} & 1.53972302 & 1.89972985 & -0.93006175 \\ \mathrm{C} & 1.86621141 & 2.09049087 & 2.55617575 \\ \mathrm{H} & 2.87843364 & 1.68564816 & 2.59833210 \\ \mathrm{H} & 1.83609107 & 2.97094306 & 3.20282316 \\ \mathrm{H} & 1.21655811 & 1.34696265 & 3.01989908 \\ \mathrm{C} & 1.72928264 & 3.91477672 & 0.69370593 \\ \mathrm{H} & 1.21815792 & 3.99704230 & -0.27230373 \\ \mathrm{C} & 3.49622822 & 1.72989577 & -0.14416566 \\ \mathrm{H} & 3.90715535 & 1.06503277 & -0.90788758 \\ \mathrm{H} & 4.02126811 & 1.47824643 & 0.78241691 \\ \mathrm{C} & 3.80325661 & 3.17327410 & -0.56868506 \\ \mathrm{H} & 3.37738441 & 3.31182348 & -1.56754128 \\ \mathrm{H} & 4.88816296 & 3.29501687 & -0.67108897 \\ \mathrm{C} & 3.24011134 & 4.24239000 & 0.40187259 \\ \mathrm{C} & 2.00518735 & -0.66215205 & -1.31980874 \\ \mathrm{H} & 3.08169776 & -0.85116806 & -1.33268735 \\ \mathrm{H} & 1.50722761 & -1.62742476 & -1.43937198 \\ \mathrm{H} & 1.75609670 & -0.04479065 & -2.19029937 \\ \mathrm{C} & 2.11952001 & -0.83569503 & 1.16268304 \\ \mathrm{H} & 1.73695669 & -0.53952488 & 2.13952606 \\ & & & \\ & & & \end{array}$




\begin{tabular}{|c|c|c|c|}
\hline $\mathrm{H}$ & 1.86443626 & -1.88817055 & 1.01959068 \\
\hline $\mathrm{H}$ & 3.21078726 & -0.76234102 & 1.19430944 \\
\hline $\mathrm{O}$ & -0.45426514 & -1.37117809 & 0.07914463 \\
\hline $\mathrm{C}$ & -1.64708209 & -1.65174225 & -0.50131031 \\
\hline $\mathrm{O}$ & -2.32876552 & -0.82880880 & -1.06110934 \\
\hline $\mathrm{C}$ & 4.10331122 & 4.21255762 & 1.68727870 \\
\hline $\mathrm{H}$ & 5.14947283 & 4.44578410 & 1.47342766 \\
\hline $\mathrm{H}$ & 3.76051816 & 4.89105293 & 2.47052829 \\
\hline $\mathrm{H}$ & 4.10702426 & 3.21614505 & 2.12185471 \\
\hline $\mathrm{C}$ & 3.31717955 & 5.70618112 & -0.28602638 \\
\hline $\mathrm{C}$ & 1.09439384 & 4.98921258 & 1.59025840 \\
\hline $\mathrm{H}$ & 0.03103034 & 4.78224353 & 1.73156971 \\
\hline $\mathrm{H}$ & 1.53991570 & 4.99870830 & 2.58936018 \\
\hline $\mathrm{C}$ & 2.68059530 & 6.74003889 & 0.69868346 \\
\hline $\mathrm{C}$ & 1.21455860 & 6.39936071 & 0.99058793 \\
\hline $\mathrm{H}$ & 0.78458781 & 7.10613923 & 1.70227526 \\
\hline $\mathrm{H}$ & 0.62160461 & 6.47645559 & 0.07031515 \\
\hline $\mathrm{C}$ & 2.60237735 & 5.71760084 & -1.66442611 \\
\hline $\mathrm{H}$ & 2.36709612 & 6.72916621 & -2.00976247 \\
\hline $\mathrm{H}$ & 3.23209199 & 5.26661148 & -2.43493756 \\
\hline $\mathrm{H}$ & 1.65821275 & 5.17686945 & -1.66466393 \\
\hline $\mathrm{H}$ & 3.22325430 & 6.69127161 & 1.64508877 \\
\hline $\mathrm{C}$ & 2.82792004 & 8.26696354 & 0.18816279 \\
\hline $\mathrm{H}$ & 2.29220268 & 8.26791147 & -0.76648697 \\
\hline $\mathrm{C}$ & 4.78655822 & 6.11298936 & -0.52632916 \\
\hline $\mathrm{H}$ & 5.36390576 & 6.09266149 & 0.40015437 \\
\hline $\mathrm{H}$ & 5.28308874 & 5.44160544 & -1.22999977 \\
\hline $\mathrm{C}$ & 4.90699267 & 7.55661632 & -1.12148227 \\
\hline $\mathrm{H}$ & 4.38724687 & 7.59595464 & -2.08297748 \\
\hline $\mathrm{C}$ & 4.24581217 & 8.45129221 & -0.16197466 \\
\hline $\mathrm{H}$ & 5.94941826 & 7.84808221 & -1.25803341 \\
\hline $\mathrm{C}$ & 2.01400283 & 9.52972873 & 3.71663050 \\
\hline $\mathrm{H}$ & 2.43725403 & 10.15774668 & 4.51346996 \\
\hline $\mathrm{C}$ & 2.20342200 & 9.36284133 & 1.10228681 \\
\hline $\mathrm{H}$ & 1.18572593 & 9.01533502 & 1.29868651 \\
\hline $\mathrm{C}$ & 2.92842549 & 9.51360123 & 2.47975162 \\
\hline $\mathrm{H}$ & 3.63227272 & 8.69070168 & 2.64348189 \\
\hline $\mathrm{H}$ & 3.50484643 & 10.45183991 & 2.49135106 \\
\hline $\mathrm{O}$ & 5.02235605 & 9.29354076 & 0.42564576 \\
\hline $\mathrm{C}$ & 1.80306670 & 8.16337229 & 4.33383987 \\
\hline $\mathrm{O}$ & 2.35672864 & 7.15644905 & 3.96023736 \\
\hline $\mathrm{H}$ & 1.10208217 & 8.14106205 & 5.19245913 \\
\hline $\mathrm{C}$ & 2.04618382 & 10.73486818 & 0.36808976 \\
\hline $\mathrm{C}$ & 1.28533366 & 11.74868670 & 1.23822525 \\
\hline $\mathrm{H}$ & 1.21224992 & 12.70764879 & 0.71856259 \\
\hline $\mathrm{H}$ & 0.26345171 & 11.40465773 & 1.43537031 \\
\hline $\mathrm{H}$ & 1.77091426 & 11.93896025 & 2.19859022 \\
\hline $\mathrm{C}$ & -1.98532863 & -3.11251373 & -0.34737195 \\
\hline $\mathrm{H}$ & -2.95219524 & -3.31061831 & -0.80777765 \\
\hline $\mathrm{H}$ & -1.21441559 & -3.72873688 & -0.81884067 \\
\hline $\mathrm{H}$ & -2.01262392 & -3.38139646 & 0.71232938 \\
\hline $\mathrm{C}$ & 1.34288802 & 10.61473224 & -0.99438352 \\
\hline $\mathrm{H}$ & 1.93693910 & 10.08617990 & -1.74558417 \\
\hline $\mathrm{H}$ & 0.37906551 & 10.10130775 & -0.89904757 \\
\hline $\mathrm{H}$ & 1.14248494 & 11.60985021 & -1.39995521 \\
\hline $\mathrm{H}$ & 3.04977272 & 11.15296430 & 0.18651479 \\
\hline $\mathrm{H}$ & 1.03101620 & 9.96898389 & 3.50888959 \\
\hline $\mathrm{H}$ & 4.56009721 & 9.80971273 & 1.11511864 \\
\hline \multicolumn{4}{|c|}{ O), $E=-1552.4069$} \\
\hline$c$ & 0.00000000 & 0.00000000 & 0.00000000 \\
\hline $\mathrm{C}$ & 1.54712224 & 0.00000000 & 0.00000000 \\
\hline
\end{tabular}




\begin{tabular}{|c|c|c|c|}
\hline $\mathrm{C}$ & 1.98496970 & 1.50694229 & 0.00000000 \\
\hline $\mathrm{C}$ & 1.39031871 & 2.42885137 & 1.12100083 \\
\hline $\mathrm{C}$ & -0.15525494 & 2.27201292 & 1.08531132 \\
\hline $\mathrm{C}$ & -0.62593418 & 0.81413268 & 1.12590202 \\
\hline $\mathrm{H}$ & -0.35108987 & 0.40394454 & -0.95565468 \\
\hline $\mathrm{H}$ & -0.61368745 & 2.80613377 & 1.92434192 \\
\hline $\mathrm{H}$ & -0.54364677 & 2.73762347 & 0.16907239 \\
\hline $\mathrm{H}$ & -0.39593963 & 0.34743208 & 2.08949443 \\
\hline $\mathrm{H}$ & -1.71318468 & 0.78273200 & 1.01359588 \\
\hline $\mathrm{H}$ & 1.54137918 & 1.90038703 & -0.92961605 \\
\hline $\mathrm{C}$ & 1.86811683 & 2.08738005 & 2.55532647 \\
\hline $\mathrm{H}$ & 2.88256674 & 1.68864393 & 2.59680456 \\
\hline $\mathrm{H}$ & 1.83354497 & 2.96575083 & 3.20579654 \\
\hline $\mathrm{H}$ & 1.22193594 & 1.33980126 & 3.01744796 \\
\hline $\mathrm{C}$ & 1.73478830 & 3.91254195 & 0.69406040 \\
\hline $\mathrm{H}$ & 1.22644048 & 3.99846831 & -0.27247173 \\
\hline $\mathrm{C}$ & 3.49721949 & 1.73036672 & -0.14285638 \\
\hline $\mathrm{H}$ & 3.90737844 & 1.06755547 & -0.90871246 \\
\hline $\mathrm{H}$ & 4.02155905 & 1.47197111 & 0.78263495 \\
\hline $\mathrm{C}$ & 3.80797940 & 3.17453499 & -0.56218435 \\
\hline $\mathrm{H}$ & 3.38721275 & 3.31791272 & -1.56203320 \\
\hline $\mathrm{H}$ & 4.89240136 & 3.29421896 & -0.66120828 \\
\hline $\mathrm{C}$ & 3.24466404 & 4.24907118 & 0.40414382 \\
\hline $\mathrm{C}$ & 2.00521371 & -0.66194028 & -1.31984024 \\
\hline $\mathrm{H}$ & 3.08183548 & -0.84977821 & -1.33272503 \\
\hline $\mathrm{H}$ & 1.50785115 & -1.62754144 & -1.43941156 \\
\hline $\mathrm{H}$ & 1.75583692 & -0.04430274 & -2.18991541 \\
\hline $\mathrm{C}$ & 2.11895662 & -0.83646683 & 1.16251959 \\
\hline $\mathrm{H}$ & 1.73390116 & -0.54295537 & 2.13934315 \\
\hline $\mathrm{H}$ & 1.86622119 & -1.88928660 & 1.01775022 \\
\hline $\mathrm{H}$ & 3.20996817 & -0.76099493 & 1.19599533 \\
\hline $\mathrm{O}$ & -0.45421800 & -1.37122969 & 0.07909454 \\
\hline $\mathrm{C}$ & -1.65131372 & -1.64983605 & -0.49347167 \\
\hline $\mathrm{O}$ & -2.33931107 & -0.82386530 & -1.04089775 \\
\hline $\mathrm{C}$ & 4.10846798 & 4.20507249 & 1.68879395 \\
\hline $\mathrm{H}$ & 5.14457027 & 4.47974884 & 1.48211238 \\
\hline $\mathrm{H}$ & 3.74845554 & 4.85422370 & 2.49182134 \\
\hline $\mathrm{H}$ & 4.13822402 & 3.20065750 & 2.10402691 \\
\hline $\mathrm{C}$ & 3.33276694 & 5.70694697 & -0.27900716 \\
\hline $\mathrm{C}$ & 1.09035683 & 4.98775032 & 1.58397449 \\
\hline $\mathrm{H}$ & 0.02750815 & 4.77714258 & 1.72288139 \\
\hline $\mathrm{H}$ & 1.52833854 & 4.97546623 & 2.59064226 \\
\hline $\mathrm{C}$ & 2.68370578 & 6.77537538 & 0.68032838 \\
\hline $\mathrm{C}$ & 1.22231734 & 6.39253299 & 0.97947473 \\
\hline $\mathrm{H}$ & 0.73915497 & 7.11407542 & 1.64923635 \\
\hline $\mathrm{H}$ & 0.64953887 & 6.45052785 & 0.04675033 \\
\hline $\mathrm{C}$ & 2.61517402 & 5.72143723 & -1.65812074 \\
\hline $\mathrm{H}$ & 2.39319568 & 6.73444195 & -1.99984626 \\
\hline $\mathrm{H}$ & 3.25023517 & 5.27084507 & -2.42414005 \\
\hline $\mathrm{H}$ & 1.66968870 & 5.18134237 & -1.66554001 \\
\hline $\mathrm{H}$ & 3.27776702 & 6.69344515 & 1.61665380 \\
\hline $\mathrm{C}$ & 2.84283918 & 8.25387917 & 0.18142318 \\
\hline $\mathrm{H}$ & 2.23697982 & 8.31887658 & -0.72784463 \\
\hline $\mathrm{C}$ & 4.80538533 & 6.12093626 & -0.52890140 \\
\hline $\mathrm{H}$ & 5.36884449 & 6.12411652 & 0.40904177 \\
\hline $\mathrm{H}$ & 5.29507513 & 5.39426227 & -1.18193795 \\
\hline $\mathrm{C}$ & 4.93854285 & 7.52143323 & -1.15342456 \\
\hline $\mathrm{H}$ & 4.46568028 & 7.54953943 & -2.14187959 \\
\hline $\mathrm{C}$ & 4.28468737 & 8.55073128 & -0.26602014 \\
\hline $\mathrm{H}$ & 5.98584628 & 7.80300208 & -1.28183412 \\
\hline $\mathrm{C}$ & 2.56642592 & 9.68366553 & 3.73822443 \\
\hline $\mathrm{H}$ & 3.22257780 & 10.12976810 & 4.51253962 \\
\hline $\mathrm{C}$ & 2.31317532 & 9.34589767 & 1.15963754 \\
\hline
\end{tabular}




$\begin{array}{cccc}\mathrm{H} & 1.340666855 & 8.99451738 & 1.53174041 \\ \mathrm{C} & 3.26580191 & 9.52974990 & 2.38416805 \\ \mathrm{H} & 3.98833135 & 8.70784577 & 2.45582302 \\ \mathrm{H} & 3.89253952 & 10.40768035 & 2.22992438 \\ \mathrm{O} & 4.87216618 & 9.54915628 & 0.09719896 \\ \mathrm{C} & 2.05470560 & 8.48502755 & 4.38581803 \\ \mathrm{O} & 2.13933111 & 7.30399629 & 3.94509409 \\ \mathrm{H} & 1.55941305 & 8.53815545 & 5.35826197 \\ \mathrm{C} & 2.01522682 & 10.70516489 & 0.45231328 \\ \mathrm{C} & 1.48900127 & 11.76731439 & 1.43298628 \\ \mathrm{H} & 1.27549123 & 12.69785728 & 0.90013899 \\ \mathrm{H} & 0.54889132 & 11.45057186 & 1.90519843 \\ \mathrm{H} & 2.20985223 & 12.02300469 & 2.21664475 \\ \mathrm{C} & -1.98457294 & -3.11279826 & -0.34980817 \\ \mathrm{H} & -2.96378579 & -3.30542776 & -0.78581152 \\ \mathrm{H} & -1.22742485 & -3.72082090 & -0.85340132 \\ \mathrm{H} & -1.98029579 & -3.39782033 & 0.70589275 \\ \mathrm{C} & 1.00843689 & 10.56015034 & -0.70061448 \\ \mathrm{H} & 1.39092710 & 9.96744459 & -1.53498898 \\ \mathrm{H} & 0.07043986 & 10.10371342 & -0.36132867 \\ \mathrm{H} & 0.76220579 & 11.54569307 & -1.10558958 \\ \mathrm{H} & 2.96277777 & 11.06931200 & 0.04344344 \\ \mathrm{H} & 1.72396181 & 10.39736078 & 3.72363183 \\ \mathrm{H} & 2.56297954 & 7.23018096 & 3.05078579\end{array}$

\section{tautomer 1, $\mathrm{H}^{+}$at $\mathrm{CH}(=\mathrm{O}), \mathrm{E}=\mathbf{- 1 5 5 2 . 3 9 0 5}$}

11

C

C

C

C

C

$\mathrm{C}$

$\mathrm{H}$

$\mathrm{H}$

$\mathrm{H}$

$\mathrm{H}$

$\mathrm{H}$

$\mathrm{H}$

C

$\mathrm{H}$

$\mathrm{H}$

$\mathrm{H}$

C

$\mathrm{H}$

C

$\mathrm{H}$

$\mathrm{H}$

C

$\mathrm{H}$

$\mathrm{H}$

C

$\mathrm{C}$

$\mathrm{H}$

$\mathrm{H}$

$\mathrm{H}$

C

$\mathrm{H}$

$\mathrm{H}$

$\mathrm{H}$

$\mathrm{O}$

$\mathrm{C}$

$\mathrm{O}$
$\begin{array}{lll}0.00000000 & 0.00000000 & 0.00000000\end{array}$ $\begin{array}{lll}1.54683433 & 0.00000000 & 0.00000000\end{array}$ $\begin{array}{lll}1.98874281 & 1.50489163 & 0.00000000\end{array}$ $\begin{array}{lll}1.39372564 & 2.43194700 & 1.11816282\end{array}$ $\begin{array}{lll}-0.15185582 & 2.27771654 & 1.07467179\end{array}$ $\begin{array}{lll}-0.62586076 & 0.82076807 & 1.12054972\end{array}$ $\begin{array}{lll}-0.35262342 & 0.39555049 & -0.95865281\end{array}$ $\begin{array}{lll}-0.61351000 & 2.81768349 & 1.90796498\end{array}$ $\begin{array}{lll}-0.53306233 & 2.74087275 & 0.15418284\end{array}$ $\begin{array}{lll}-0.39582671 & 0.35812932 & 2.08617602\end{array}$ $\begin{array}{lll}-1.71321297 & 0.78962315 & 1.00825218\end{array}$ $\begin{array}{lll}1.55072830 & 1.89966461 & -0.93149386\end{array}$ $\begin{array}{lll}1.86438892 & 2.08628709 & 2.55403438\end{array}$ $\begin{array}{lll}2.87413458 & 1.67615737 & 2.59592117\end{array}$ $\begin{array}{lll}1.83982606 & 2.96695505 & 3.20178823\end{array}$ $\begin{array}{lll}1.20990708 & 1.34615972 & 3.01748216\end{array}$ $\begin{array}{lll}1.74666818 & 3.91425578 & 0.69341605\end{array}$ $1.24801628 \quad 4.00266134-0.27830401$ $\begin{array}{llll}3.50289020 & 1.72032904 & -0.14104758\end{array}$ $\begin{array}{llll}3.91060613 & 1.05535830 & -0.90661178\end{array}$ $\begin{array}{llll}4.02596907 & 1.45990206 & 0.78525548\end{array}$ $\begin{array}{llll}3.81548693 & 3.16315915 & -0.55926856\end{array}$ $\begin{array}{llll}3.38970344 & 3.31172289 & -1.55662774\end{array}$ $\begin{array}{lll}4.90003458 & 3.27933683 & -0.66504660\end{array}$ $\begin{array}{lll}3.26085215 & 4.23668972 & 0.41178936\end{array}$ $\begin{array}{llll}2.00342926 & -0.66606660 & -1.31812405\end{array}$ $\begin{array}{llll}3.08027183 & -0.85227290 & -1.33296107\end{array}$ $\begin{array}{lll}1.50706525 & -1.63280778 & -1.43325697\end{array}$ $\begin{array}{lll}1.75117535 & -0.05222522 & -2.18998778\end{array}$ $\begin{array}{lll}2.11634581 & -0.83580493 & 1.16414901\end{array}$ $\begin{array}{lll}1.71936630 & -0.54948773 & 2.13859143\end{array}$ $\begin{array}{lll}1.87490709 & -1.89052836 & 1.01379465\end{array}$ $\begin{array}{lll}3.20627783 & -0.74966189 & 1.20750182\end{array}$ $\begin{array}{lll}-0.45071845 & -1.37301331 & 0.09115665\end{array}$ $\begin{array}{lll}-1.64607455 & -1.66388377 & -0.47576609\end{array}$ $-2.34114479-0.84946745-1.03171588$ 


\begin{tabular}{|c|c|c|c|}
\hline $\mathrm{C}$ & 4.12612307 & 4.18253818 & 1.69507900 \\
\hline $\mathrm{H}$ & 5.16684363 & 4.42985831 & 1.47509021 \\
\hline $\mathrm{H}$ & 3.78501049 & 4.85918761 & 2.48281945 \\
\hline $\mathrm{H}$ & 4.13288875 & 3.18353646 & 2.12398610 \\
\hline $\mathrm{C}$ & 3.36143327 & 5.69229228 & -0.25785602 \\
\hline $\mathrm{C}$ & 1.10299598 & 5.00139550 & 1.57201589 \\
\hline $\mathrm{H}$ & 0.03983243 & 4.79195362 & 1.71345516 \\
\hline $\mathrm{H}$ & 1.54634933 & 5.00638347 & 2.57661779 \\
\hline $\mathrm{C}$ & 2.71882877 & 6.75489758 & 0.70001459 \\
\hline $\mathrm{C}$ & 1.24167202 & 6.40206195 & 0.95759518 \\
\hline $\mathrm{H}$ & 0.77342148 & 7.12117294 & 1.63827025 \\
\hline $\mathrm{H}$ & 0.66923419 & 6.46146147 & 0.02548375 \\
\hline $\mathrm{C}$ & 2.66201124 & 5.73045132 & -1.64466479 \\
\hline $\mathrm{H}$ & 2.44962949 & 6.75870126 & -1.94633247 \\
\hline $\mathrm{H}$ & 3.29917142 & 5.29264208 & -2.41750628 \\
\hline $\mathrm{H}$ & 1.71358499 & 5.19534023 & -1.67142374 \\
\hline $\mathrm{H}$ & 3.21855558 & 6.63542311 & 1.67696709 \\
\hline $\mathrm{C}$ & 2.99223677 & 8.20638142 & 0.28549066 \\
\hline $\mathrm{C}$ & 4.83108667 & 6.11979657 & -0.47942278 \\
\hline $\mathrm{H}$ & 5.35062595 & 6.19737185 & 0.47977754 \\
\hline $\mathrm{H}$ & 5.36856942 & 5.37627926 & -1.07368669 \\
\hline $\mathrm{C}$ & 4.92800774 & 7.48263946 & -1.17321293 \\
\hline $\mathrm{H}$ & 4.70128582 & 7.40311285 & -2.24512585 \\
\hline $\mathrm{C}$ & 4.02052118 & 8.49621316 & -0.54354583 \\
\hline $\mathrm{H}$ & 5.96146053 & 7.85174917 & -1.11074079 \\
\hline $\mathrm{C}$ & 2.62172892 & 10.45078480 & 3.28353616 \\
\hline $\mathrm{H}$ & 3.25836845 & 11.19373791 & 3.81361976 \\
\hline $\mathrm{C}$ & 2.23378827 & 9.33731148 & 0.97282287 \\
\hline $\mathrm{H}$ & 1.49360236 & 8.87455757 & 1.63508463 \\
\hline $\mathrm{C}$ & 3.20917540 & 10.15417401 & 1.89737104 \\
\hline $\mathrm{H}$ & 4.14657329 & 9.61012032 & 2.02674382 \\
\hline $\mathrm{H}$ & 3.47126311 & 11.10538970 & 1.43083706 \\
\hline $\mathrm{O}$ & 4.30887532 & 9.80686195 & -0.81464338 \\
\hline $\mathrm{C}$ & 2.55585247 & 9.35951945 & 4.24015311 \\
\hline $\mathrm{O}$ & 3.24276582 & 8.29153391 & 4.05385769 \\
\hline $\mathrm{H}$ & 1.95688225 & 9.43299707 & 5.15016852 \\
\hline $\mathrm{C}$ & 1.42307408 & 10.26313980 & 0.01274626 \\
\hline $\mathrm{C}$ & 0.66302251 & 11.36581846 & 0.76453298 \\
\hline $\mathrm{H}$ & 0.08791680 & 11.97489647 & 0.06102386 \\
\hline $\mathrm{H}$ & -0.05629625 & 10.94334403 & 1.47911274 \\
\hline $\mathrm{H}$ & 1.32462367 & 12.05542224 & 1.30023281 \\
\hline $\mathrm{C}$ & -1.96914701 & -3.12803929 & -0.31590959 \\
\hline $\mathrm{H}$ & -2.95243785 & -3.33044017 & -0.73813032 \\
\hline $\mathrm{H}$ & -1.21470104 & -3.73507761 & -0.82492096 \\
\hline $\mathrm{H}$ & -1.94905489 & -3.40521703 & 0.74166366 \\
\hline $\mathrm{C}$ & 0.43643220 & 9.45807719 & -0.84367073 \\
\hline $\mathrm{H}$ & 0.94322092 & 8.68903774 & -1.43100984 \\
\hline $\mathrm{H}$ & -0.32237651 & 8.96619240 & -0.22360487 \\
\hline $\mathrm{H}$ & -0.08873564 & 10.11822774 & -1.54033572 \\
\hline $\mathrm{H}$ & 2.14165913 & 10.74441007 & -0.65685823 \\
\hline $\mathrm{H}$ & 1.63743978 & 10.93870356 & 3.26959887 \\
\hline $\mathrm{H}$ & 5.04074396 & 9.85175831 & -1.44083807 \\
\hline 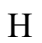 & 3.15868856 & 7.64198319 & 4.77466353 \\
\hline
\end{tabular}

\begin{tabular}{lrrr}
\multicolumn{4}{l}{ tautomer $\mathbf{2}, \mathbf{H}^{+}$at $\mathbf{C H}(=\mathbf{O}), \mathbf{E}=\mathbf{- 1 5 5 2 . 3 8 6 4}$} \\
11 & & & \\
$\mathrm{C}$ & 0.00000000 & 0.00000000 & 0.00000000 \\
$\mathrm{C}$ & 1.54677895 & 0.00000000 & 0.00000000 \\
$\mathrm{C}$ & 1.99018105 & 1.50473672 & 0.00000000 \\
$\mathrm{C}$ & 1.38909759 & 2.43672037 & 1.10980561 \\
$\mathrm{C}$ & -0.15629414 & 2.27588296 & 1.07188166 \\
$\mathrm{C}$ & -0.62588801 & 0.81800239 & 1.12250864 \\
$\mathrm{H}$ & -0.35218860 & 0.39902761 & -0.95724734
\end{tabular}




\begin{tabular}{|c|c|c|c|}
\hline $\mathrm{H}$ & -0.61779349 & 2.81909067 & 1.90320543 \\
\hline $\mathrm{H}$ & -0.54186110 & 2.73325305 & 0.15052194 \\
\hline 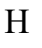 & -0.39317850 & 0.35627132 & 2.08795766 \\
\hline $\mathrm{H}$ & -1.71340951 & 0.78379485 & 1.01227307 \\
\hline $\mathrm{H}$ & 1.56099624 & 1.89755035 & -0.93640310 \\
\hline $\mathrm{C}$ & 1.86389556 & 2.10118239 & 2.54613567 \\
\hline $\mathrm{H}$ & 2.89269300 & 1.74118326 & 2.59471454 \\
\hline $\mathrm{H}$ & 1.78791321 & 2.97323418 & 3.20153900 \\
\hline $\mathrm{H}$ & 1.24242414 & 1.32591745 & 2.99669211 \\
\hline $\mathrm{C}$ & 1.72976643 & 3.91904244 & 0.67498204 \\
\hline $\mathrm{H}$ & 1.20160604 & 4.01260195 & -0.28026584 \\
\hline $\mathrm{C}$ & 3.50633079 & 1.71937371 & -0.12574829 \\
\hline $\mathrm{H}$ & 3.92742608 & 1.03830313 & -0.86975158 \\
\hline $\mathrm{H}$ & 4.01779234 & 1.48281788 & 0.81338840 \\
\hline $\mathrm{C}$ & 3.81556617 & 3.15306456 & -0.57525889 \\
\hline $\mathrm{H}$ & 3.41128937 & 3.26401788 & -1.58533920 \\
\hline $\mathrm{H}$ & 4.90052283 & 3.28281101 & -0.66233077 \\
\hline $\mathrm{C}$ & 3.23229516 & 4.25128598 & 0.35058123 \\
\hline $\mathrm{C}$ & 2.00368007 & -0.66521142 & -1.31853182 \\
\hline $\mathrm{H}$ & 3.08007127 & -0.85425677 & -1.33191373 \\
\hline $\mathrm{H}$ & 1.50510131 & -1.63049023 & -1.43621221 \\
\hline $\mathrm{H}$ & 1.75469229 & -0.04921173 & -2.18970423 \\
\hline $\mathrm{C}$ & 2.11647474 & -0.83675600 & 1.16332409 \\
\hline $\mathrm{H}$ & 1.72460226 & -0.54709554 & 2.13882930 \\
\hline $\mathrm{H}$ & 1.86947130 & -1.89068120 & 1.01574662 \\
\hline $\mathrm{H}$ & 3.20691583 & -0.75572148 & 1.20267722 \\
\hline $\mathrm{O}$ & -0.45148799 & -1.37311282 & 0.08558052 \\
\hline $\mathrm{C}$ & -1.64472377 & -1.66082522 & -0.48785097 \\
\hline $\mathrm{O}$ & -2.33615472 & -0.84382817 & -1.04445085 \\
\hline $\mathrm{C}$ & 4.09957284 & 4.25886012 & 1.63593444 \\
\hline $\mathrm{H}$ & 5.13892710 & 4.51385002 & 1.41699361 \\
\hline $\mathrm{H}$ & 3.74478402 & 4.95445811 & 2.40027874 \\
\hline $\mathrm{H}$ & 4.11842195 & 3.27273566 & 2.09570985 \\
\hline $\mathrm{C}$ & 3.28818098 & 5.68260655 & -0.38072800 \\
\hline $\mathrm{C}$ & 1.12770143 & 5.00069093 & 1.59141759 \\
\hline $\mathrm{H}$ & 0.06824023 & 4.79987943 & 1.77057278 \\
\hline $\mathrm{H}$ & 1.60511688 & 4.98294875 & 2.57891318 \\
\hline $\mathrm{C}$ & 2.69445905 & 6.76808340 & 0.57764796 \\
\hline $\mathrm{C}$ & 1.25602842 & 6.40569840 & 0.99149471 \\
\hline $\mathrm{H}$ & 0.86480374 & 7.13574203 & 1.71032012 \\
\hline $\mathrm{H}$ & 0.60109734 & 6.48510266 & 0.11569077 \\
\hline $\mathrm{C}$ & 2.51106798 & 5.69124669 & -1.72398511 \\
\hline $\mathrm{H}$ & 2.59012354 & 6.67137336 & -2.19936535 \\
\hline $\mathrm{H}$ & 2.92963297 & 4.97467465 & -2.43258484 \\
\hline $\mathrm{H}$ & 1.44953175 & 5.46616293 & -1.62185698 \\
\hline $\mathrm{H}$ & 3.31828085 & 6.75656595 & 1.48070817 \\
\hline $\mathrm{C}$ & 2.75736843 & 8.22124633 & 0.02348080 \\
\hline $\mathrm{H}$ & 1.91171728 & 8.35708363 & -0.66078510 \\
\hline $\mathrm{C}$ & 4.74975740 & 6.07746098 & -0.72377339 \\
\hline $\mathrm{H}$ & 5.42747866 & 5.86131004 & 0.10975584 \\
\hline $\mathrm{H}$ & 5.10891635 & 5.45810721 & -1.55328210 \\
\hline $\mathrm{C}$ & 4.89651876 & 7.52403074 & -1.10487910 \\
\hline $\mathrm{C}$ & 4.00436057 & 8.46669478 & -0.78871577 \\
\hline $\mathrm{H}$ & 5.76773366 & 7.80035569 & -1.69771434 \\
\hline $\mathrm{C}$ & 3.92836958 & 9.98857897 & 3.28443046 \\
\hline $\mathrm{H}$ & 4.86254492 & 10.49085847 & 3.61959719 \\
\hline $\mathrm{C}$ & 2.58079741 & 9.28994031 & 1.16871927 \\
\hline $\mathrm{H}$ & 1.97194187 & 8.81179865 & 1.94811664 \\
\hline $\mathrm{C}$ & 3.96322450 & 9.62181398 & 1.79984644 \\
\hline $\mathrm{H}$ & 4.64192803 & 8.77292631 & 1.66759960 \\
\hline $\mathrm{H}$ & 4.42353538 & 10.45295471 & 1.26241716 \\
\hline $\mathrm{O}$ & 4.11640690 & 9.77626419 & -1.18737587 \\
\hline $\mathrm{C}$ & 3.76476654 & 8.94259529 & 4.27217491 \\
\hline
\end{tabular}




$\begin{array}{cccc}\mathrm{O} & 3.75514868 & 7.71191678 & 3.93937821 \\ \mathrm{H} & 3.66322044 & 9.18868342 & 5.33291463 \\ \mathrm{C} & 1.77829879 & 10.53732230 & 0.68403299 \\ \mathrm{C} & 1.83265080 & 11.74663895 & 1.63165470 \\ \mathrm{H} & 1.26207878 & 12.57776266 & 1.20694555 \\ \mathrm{H} & 1.37176630 & 11.52487990 & 2.60466176 \\ \mathrm{H} & 2.84876814 & 12.12216438 & 1.79320910 \\ \mathrm{C} & -1.97080416 & -3.12500526 & -0.33451622 \\ \mathrm{H} & -2.95240966 & -3.32411210 & -0.76222739 \\ \mathrm{H} & -1.21506007 & -3.73160770 & -0.84209130 \\ \mathrm{H} & -1.95642596 & -3.40587907 & 0.72216481 \\ \mathrm{C} & 0.30262990 & 10.18122680 & 0.43833551 \\ \mathrm{H} & 0.17427549 & 9.35584919 & -0.26567308 \\ \mathrm{H} & -0.19411806 & 9.89905712 & 1.37487608 \\ \mathrm{H} & -0.23381576 & 11.04228402 & 0.02938971 \\ \mathrm{H} & 2.22248036 & 10.85087957 & -0.26550894 \\ \mathrm{H} & 3.17772871 & 10.75675411 & 3.54031836 \\ \mathrm{H} & 4.87189419 & 9.86223601 & -1.78273188 \\ \mathrm{H} & 3.65683302 & 7.09376290 & 4.68761667\end{array}$

\section{tautomer $3, \mathrm{H}^{+}$at $\mathrm{C}=\mathrm{O}, \mathrm{E}=\mathbf{- 1 5 5 2 . 4 1 3 8}$}

11

C

0.00000000

1.54731592

$\begin{array}{ll}0.00000000 & 0.00000000\end{array}$

$\begin{array}{lll}1.98823543 & 1.50589220 & 0.00000000\end{array}$

$\begin{array}{lll}1.39535544 & 2.42930670 & 1.12113901\end{array}$

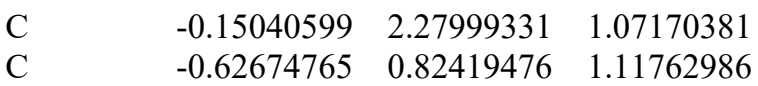

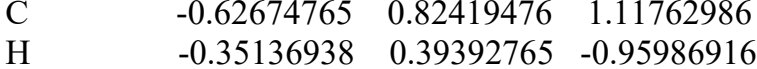

$\begin{array}{llll}\mathrm{H} & -0.61391295 & 2.82231493 & 1.90214403\end{array}$

$\begin{array}{llll}\mathrm{H} & -0.52763895 & 2.74125944 & 0.14840452\end{array}$

$\begin{array}{llll}\mathrm{H} & -0.40356736 & 0.36257625 & 2.08519326\end{array}$

$\begin{array}{llll}\mathrm{H} & -1.71374253 & 0.79689712 & 1.00131994\end{array}$

$\begin{array}{llll}\mathrm{H} & 1.54599837 & 1.89962762 & -0.93044767\end{array}$

$\begin{array}{llll}\text { C } & 1.86057504 & 2.07787855 & 2.55742071\end{array}$

$\begin{array}{llll}\mathrm{H} & 2.86706957 & 1.65966104 & 2.60213834\end{array}$

$\begin{array}{llll}\mathrm{H} & 1.83803420 & 2.95347998 & 3.21088820\end{array}$

$\begin{array}{llll}\mathrm{H} & 1.20007997 & 1.33923035 & 3.01358929\end{array}$

$\begin{array}{llll}\text { C } & 1.74677271 & 3.91615579 & 0.70545309\end{array}$

$\begin{array}{llll}\mathrm{H} & 1.23858370 & 4.01151035 & -0.26100184\end{array}$

$\begin{array}{lllll}\text { C } & 3.50142178 & 1.72238742 & -0.14610586\end{array}$

$\begin{array}{llll}\mathrm{H} & 3.90698875 & 1.05876461 & -0.91369835\end{array}$

$\begin{array}{llll}\mathrm{H} & 4.02968211 & 1.46489013 & 0.77757849\end{array}$

$\begin{array}{lllll}\text { C } & 3.80981396 & 3.16603707 & -0.56698558\end{array}$

$\mathrm{H} \quad 3.37299017 \quad 3.31249928-1.56010315$

$\begin{array}{llll}\mathrm{H} & 4.89413729 & 3.28504833 & -0.68091159\end{array}$

$\begin{array}{llll}\mathrm{C} & 3.26024369 & 4.23160083 & 0.41541687\end{array}$

$\begin{array}{llll}\mathrm{C} & 2.00412513 & -0.66274662 & -1.32011602\end{array}$

$\mathrm{H} \quad 3.08035708 \quad-0.85383601-1.33428955$

$\mathrm{H} \quad \begin{array}{llll}3.50485253 & -1.62741482 & -1.43915598\end{array}$

$\begin{array}{llll}\mathrm{H} & 1.75503604 & -0.04593027 & -2.19105527\end{array}$

$\begin{array}{llll}\mathrm{C} & 2.11926101 & -0.83766994 & 1.16144756\end{array}$

$\begin{array}{llll}\mathrm{H} & 1.73279046 & -0.54843728 & 2.13922476\end{array}$

$\begin{array}{llll}\mathrm{H} & 1.86840599 & -1.89030486 & 1.01310387\end{array}$

$\begin{array}{llll}\mathrm{H} & 3.21027469 & -0.76084781 & 1.19609443\end{array}$

$\begin{array}{llll}\mathrm{O} & -0.45055412 & -1.37108755 & 0.09303873\end{array}$

$\begin{array}{lllll}\text { C } & -1.64363231 & -1.66210341 & -0.48232080\end{array}$

$\begin{array}{lllll}\mathrm{O} & -2.33015032 & -0.84682127 & -1.04703324\end{array}$

$\begin{array}{llll}\text { C } & 4.12761112 & 4.17442148 & 1.69682129\end{array}$

$\begin{array}{llll}\mathrm{H} & 5.18162802 & 4.36385811 & 1.47778822\end{array}$

$\begin{array}{llll}\mathrm{H} & 3.81580038 & 4.86839218 & 2.47981297\end{array}$

$\begin{array}{llll}\mathrm{H} & 4.09222534 & 3.18185123 & 2.13750307\end{array}$

$\begin{array}{lllll}\text { C } & 3.35493785 & 5.70319403 & -0.25563462\end{array}$ 


\begin{tabular}{|c|c|c|c|}
\hline $\mathrm{C}$ & 1.11835921 & 4.99185492 & 1.60633891 \\
\hline $\mathrm{H}$ & 0.05291099 & 4.79288871 & 1.74380158 \\
\hline $\mathrm{H}$ & 1.56022385 & 4.98628809 & 2.60852727 \\
\hline $\mathrm{C}$ & 2.72562892 & 6.72936886 & 0.73801439 \\
\hline $\mathrm{C}$ & 1.25605106 & 6.40437239 & 1.01656565 \\
\hline $\mathrm{H}$ & 0.83556641 & 7.11494426 & 1.72914568 \\
\hline $\mathrm{H}$ & 0.67019053 & 6.49362060 & 0.09319152 \\
\hline $\mathrm{C}$ & 2.64478590 & 5.73931753 & -1.63561716 \\
\hline $\mathrm{H}$ & 2.42465792 & 6.75899923 & -1.96863197 \\
\hline $\mathrm{H}$ & 3.26810131 & 5.28571062 & -2.41025188 \\
\hline $\mathrm{H}$ & 1.69194197 & 5.21345604 & -1.64224246 \\
\hline $\mathrm{H}$ & 3.26470247 & 6.66096310 & 1.68528099 \\
\hline $\mathrm{C}$ & 2.89342481 & 8.26767737 & 0.27241712 \\
\hline $\mathrm{H}$ & 2.35341763 & 8.30418703 & -0.68347187 \\
\hline $\mathrm{C}$ & 4.82910225 & 6.09525806 & -0.48328533 \\
\hline $\mathrm{H}$ & 5.40064314 & 6.05659897 & 0.44653991 \\
\hline $\mathrm{H}$ & 5.32235220 & 5.42789823 & -1.19323363 \\
\hline $\mathrm{C}$ & 4.95739156 & 7.54388629 & -1.05807783 \\
\hline $\mathrm{H}$ & 4.44296206 & 7.59699798 & -2.02266468 \\
\hline $\mathrm{C}$ & 4.29888282 & 8.45266930 & -0.10352960 \\
\hline $\mathrm{H}$ & 6.00653739 & 7.81860066 & -1.21374382 \\
\hline $\mathrm{C}$ & 2.12577748 & 8.44309214 & 3.65150639 \\
\hline $\mathrm{H}$ & 1.06952150 & 8.66641635 & 3.80330689 \\
\hline $\mathrm{C}$ & 2.28734471 & 9.34068658 & 1.22155083 \\
\hline $\mathrm{H}$ & 1.22822652 & 9.07300047 & 1.28709805 \\
\hline $\mathrm{C}$ & 2.87495657 & 9.30420697 & 2.67079220 \\
\hline $\mathrm{H}$ & 3.92381910 & 8.99169474 & 2.65432782 \\
\hline $\mathrm{H}$ & 2.89382782 & 10.33529560 & 3.04184128 \\
\hline $\mathrm{O}$ & 4.98562299 & 9.36658281 & 0.49414180 \\
\hline $\mathrm{C}$ & 2.69813134 & 7.47317737 & 4.37044259 \\
\hline $\mathrm{O}$ & 2.11397161 & 6.66778988 & 5.28945302 \\
\hline $\mathrm{H}$ & 3.75407797 & 7.23121564 & 4.27574215 \\
\hline $\mathrm{C}$ & 2.30805064 & 10.79017970 & 0.63348504 \\
\hline $\mathrm{C}$ & 1.23646390 & 11.64646129 & 1.32956299 \\
\hline $\mathrm{H}$ & 1.31033565 & 12.68900540 & 1.00785600 \\
\hline $\mathrm{H}$ & 0.23141283 & 11.29093150 & 1.07464295 \\
\hline $\mathrm{H}$ & 1.32804711 & 11.63328909 & 2.41873802 \\
\hline $\mathrm{C}$ & -1.97203776 & -3.12394954 & -0.31836235 \\
\hline $\mathrm{H}$ & -2.95526204 & -3.32407994 & -0.74173623 \\
\hline $\mathrm{H}$ & -1.21910878 & -3.73521513 & -0.82464598 \\
\hline $\mathrm{H}$ & -1.95444637 & -3.39798135 & 0.74000149 \\
\hline $\mathrm{C}$ & 2.09040273 & 10.86078084 & -0.88597607 \\
\hline $\mathrm{H}$ & 2.90569133 & 10.41155630 & -1.46529441 \\
\hline $\mathrm{H}$ & 1.15638119 & 10.36796238 & -1.18272927 \\
\hline $\mathrm{H}$ & 2.01998865 & 11.90376633 & -1.20665101 \\
\hline $\mathrm{H}$ & 3.28679884 & 11.23393486 & 0.85492395 \\
\hline $\mathrm{H}$ & 1.19287739 & 6.93009008 & 5.41676815 \\
\hline $\mathrm{H}$ & 5.91070822 & 9.39541858 & 0.18934044 \\
\hline
\end{tabular}

\begin{tabular}{|c|c|c|c|}
\hline $\mathrm{C}$ & 0.00000000 & 0.00000000 & 0.00000000 \\
\hline $\mathrm{C}$ & 1.54722523 & 0.00000000 & 0.00000000 \\
\hline $\mathrm{C}$ & 1.98566884 & 1.50683402 & 0.00000000 \\
\hline $\mathrm{C}$ & 1.38929273 & 2.42946433 & 1.12020154 \\
\hline $\mathrm{C}$ & -0.15632422 & 2.27389389 & 1.08083335 \\
\hline $\mathrm{C}$ & -0.62658089 & 0.81613036 & 1.12364645 \\
\hline $\mathrm{H}$ & -0.35090983 & 0.40123456 & -0.95680621 \\
\hline $\mathrm{H}$ & -0.61568568 & 2.81099337 & 1.91685597 \\
\hline $\mathrm{H}$ & -0.54237066 & 2.73711360 & 0.16223376 \\
\hline $\mathrm{H}$ & -0.39820954 & 0.35137493 & 2.08837445 \\
\hline $\mathrm{H}$ & -1.71381791 & 0.78485748 & 1.01029588 \\
\hline $\mathrm{H}$ & 1.54487662 & 1.89952977 & -0.93147672 \\
\hline
\end{tabular}




\begin{tabular}{|c|c|c|c|}
\hline $\mathrm{C}$ & 1.86387434 & 2.09031145 & 2.55541851 \\
\hline $\mathrm{H}$ & 2.87978786 & 1.69559609 & 2.59871037 \\
\hline $\mathrm{H}$ & 1.82296379 & 2.96827519 & 3.20500467 \\
\hline $\mathrm{H}$ & 1.22093319 & 1.33881485 & 3.01558152 \\
\hline $\mathrm{C}$ & 1.73075097 & 3.91530552 & 0.69404992 \\
\hline $\mathrm{H}$ & 1.21906096 & 3.99932788 & -0.27122897 \\
\hline $\mathrm{C}$ & 3.49886933 & 1.72805784 & -0.13963944 \\
\hline $\mathrm{H}$ & 3.91156231 & 1.06150467 & -0.90071366 \\
\hline $\mathrm{H}$ & 4.02219059 & 1.47869833 & 0.78873916 \\
\hline $\mathrm{C}$ & 3.80738167 & 3.17078789 & -0.56647888 \\
\hline $\mathrm{H}$ & 3.38447656 & 3.30843307 & -1.56673740 \\
\hline $\mathrm{H}$ & 4.89312768 & 3.29184137 & -0.66565379 \\
\hline $\mathrm{C}$ & 3.24186170 & 4.24088956 & 0.40148896 \\
\hline $\mathrm{C}$ & 2.00339725 & -0.66283522 & -1.31993971 \\
\hline $\mathrm{H}$ & 3.08124756 & -0.84413716 & -1.33941655 \\
\hline $\mathrm{H}$ & 1.51176422 & -1.63203299 & -1.43360241 \\
\hline $\mathrm{H}$ & 1.74324327 & -0.04962679 & -2.19013833 \\
\hline $\mathrm{C}$ & 2.11885662 & -0.83523381 & 1.16366268 \\
\hline $\mathrm{H}$ & 1.73588501 & -0.53743625 & 2.13976647 \\
\hline $\mathrm{H}$ & 1.86263071 & -1.88763049 & 1.02216009 \\
\hline $\mathrm{H}$ & 3.21033256 & -0.76320693 & 1.19534178 \\
\hline $\mathrm{O}$ & -0.45309130 & -1.37095108 & 0.08343934 \\
\hline $\mathrm{C}$ & -1.64433258 & -1.65545150 & -0.49941317 \\
\hline $\mathrm{O}$ & -2.32248629 & -0.83661790 & -1.06914501 \\
\hline $\mathrm{C}$ & 4.10544463 & 4.21473006 & 1.68721124 \\
\hline $\mathrm{H}$ & 5.15337458 & 4.43835774 & 1.46924763 \\
\hline $\mathrm{H}$ & 3.76865429 & 4.89786819 & 2.46962053 \\
\hline $\mathrm{H}$ & 4.10321434 & 3.22130878 & 2.12854961 \\
\hline $\mathrm{C}$ & 3.31620877 & 5.70465263 & -0.28993459 \\
\hline $\mathrm{C}$ & 1.09858202 & 4.99196793 & 1.59013495 \\
\hline $\mathrm{H}$ & 0.03670707 & 4.78424395 & 1.74016364 \\
\hline $\mathrm{H}$ & 1.55124663 & 4.99990530 & 2.58717645 \\
\hline $\mathrm{C}$ & 2.67939597 & 6.73761313 & 0.69272600 \\
\hline $\mathrm{C}$ & 1.21310033 & 6.40101870 & 0.98587327 \\
\hline $\mathrm{H}$ & 0.79107668 & 7.11253979 & 1.69647627 \\
\hline $\mathrm{H}$ & 0.62254349 & 6.47170068 & 0.06391897 \\
\hline $\mathrm{C}$ & 2.60330914 & 5.71549048 & -1.66936701 \\
\hline $\mathrm{H}$ & 2.34787181 & 6.72623678 & -2.00343217 \\
\hline $\mathrm{H}$ & 3.24091315 & 5.28146552 & -2.44342632 \\
\hline $\mathrm{H}$ & 1.66945026 & 5.15764719 & -1.67523848 \\
\hline $\mathrm{H}$ & 3.22600435 & 6.68204184 & 1.63742811 \\
\hline $\mathrm{C}$ & 2.83151287 & 8.27194501 & 0.19906555 \\
\hline $\mathrm{H}$ & 2.29284901 & 8.28680164 & -0.75627987 \\
\hline $\mathrm{C}$ & 4.78570354 & 6.11041799 & -0.53099224 \\
\hline $\mathrm{H}$ & 5.36349114 & 6.08855302 & 0.39562213 \\
\hline $\mathrm{H}$ & 5.28331759 & 5.44137852 & -1.23624620 \\
\hline $\mathrm{C}$ & 4.89712049 & 7.55590047 & -1.12445098 \\
\hline $\mathrm{H}$ & 4.37220983 & 7.59513278 & -2.08295336 \\
\hline $\mathrm{C}$ & 4.24102521 & 8.45205219 & -0.15911249 \\
\hline $\mathrm{H}$ & 5.94350337 & 7.83505253 & -1.28883734 \\
\hline $\mathrm{C}$ & 1.82561593 & 8.70651555 & 3.59865020 \\
\hline $\mathrm{H}$ & 0.85968494 & 9.19259971 & 3.69855483 \\
\hline $\mathrm{C}$ & 2.21806133 & 9.34801530 & 1.13583466 \\
\hline $\mathrm{H}$ & 1.15772879 & 9.08327792 & 1.18377977 \\
\hline $\mathrm{C}$ & 2.78270899 & 9.29667136 & 2.59800187 \\
\hline $\mathrm{H}$ & 3.73266921 & 8.75034823 & 2.61135444 \\
\hline $\mathrm{H}$ & 3.04672944 & 10.31636017 & 2.89979026 \\
\hline $\mathrm{O}$ & 4.93488682 & 9.32877410 & 0.48674641 \\
\hline $\mathrm{C}$ & 2.03965725 & 7.64159878 & 4.37562156 \\
\hline $\mathrm{O}$ & 3.16800624 & 6.87546355 & 4.44985869 \\
\hline $\mathrm{H}$ & 1.27860500 & 7.26132208 & 5.04868480 \\
\hline $\mathrm{C}$ & 2.25627965 & 10.79446986 & 0.55012978 \\
\hline$C$ & 1.24545539 & 11.68024240 & 1.29738654 \\
\hline
\end{tabular}




$\begin{array}{lrrr}\mathrm{H} & 1.32929899 & 12.71949748 & 0.96792398 \\ \mathrm{H} & 0.21927573 & 11.35053784 & 1.09847500 \\ \mathrm{H} & 1.39572829 & 11.66823862 & 2.38028739 \\ \mathrm{C} & -1.98465852 & -3.11434030 & -0.33374476 \\ \mathrm{H} & -2.95103533 & -3.31509352 & -0.79400371 \\ \mathrm{H} & -1.21391378 & -3.73549642 & -0.79901686 \\ \mathrm{H} & -2.01379592 & -3.37435038 & 0.72810609 \\ \mathrm{C} & 1.97132861 & 10.86655349 & -0.95808632 \\ \mathrm{H} & 2.75634343 & 10.41062888 & -1.57282942 \\ \mathrm{H} & 1.02072436 & 10.38192736 & -1.21228845 \\ \mathrm{H} & 1.89661925 & 11.91001939 & -1.27582820 \\ \mathrm{H} & 3.25679455 & 11.20965374 & 0.72520530 \\ \mathrm{H} & 3.91206343 & 7.35378429 & 4.06636889 \\ \mathrm{H} & 5.86482245 & 9.35355463 & 0.19683132\end{array}$

Structures with hydrogen bonds and similar weak interactions

\begin{tabular}{lrrr} 
IV (Fig. 5S) E $=\mathbf{- 1 5 5 2 . 4 3 7 0}$ & & \\
$1 \mathrm{H}$ & & & \\
$\mathrm{C}$ & 0.00000000 & 0.00000000 & 0.00000000 \\
$\mathrm{C}$ & 1.54689266 & 0.00000000 & 0.00000000 \\
$\mathrm{C}$ & 1.98581181 & 1.50654978 & 0.00000000 \\
$\mathrm{C}$ & 1.39154383 & 2.43005904 & 1.12089667 \\
$\mathrm{C}$ & -0.15422359 & 2.27322435 & 1.08348265 \\
$\mathrm{C}$ & -0.62503961 & 0.81506884 & 1.12518348 \\
$\mathrm{H}$ & -0.35052811 & 0.40336609 & -0.95620898 \\
$\mathrm{H}$ & -0.61354353 & 2.80892048 & 1.92083808 \\
$\mathrm{H}$ & -0.54190237 & 2.73701091 & 0.16590631 \\
$\mathrm{H}$ & -0.39504000 & 0.34907525 & 2.08904451 \\
$\mathrm{H}$ & -1.71238780 & 0.78406089 & 1.01355301 \\
$\mathrm{H}$ & 1.54424056 & 1.90062806 & -0.93030333 \\
$\mathrm{C}$ & 1.86870323 & 2.08829399 & 2.55495843 \\
$\mathrm{H}$ & 2.88321905 & 1.68998805 & 2.59664148 \\
$\mathrm{H}$ & 1.83272519 & 2.96530209 & 3.20672551 \\
$\mathrm{H}$ & 1.22273015 & 1.33983868 & 3.01562400 \\
$\mathrm{C}$ & 1.73381039 & 3.91511033 & 0.69468843 \\
$\mathrm{H}$ & 1.22187991 & 4.00096871 & -0.26962892 \\
$\mathrm{C}$ & 3.49926971 & 1.72446974 & -0.14209768 \\
$\mathrm{H}$ & 3.90829438 & 1.05967149 & -0.90664915 \\
$\mathrm{H}$ & 4.02387764 & 1.46727620 & 0.78357489 \\
$\mathrm{C}$ & 3.81103141 & 3.16637210 & -0.56560592 \\
$\mathrm{H}$ & 3.39258628 & 3.30411652 & -1.56735578 \\
$\mathrm{H}$ & 4.89660387 & 3.28733241 & -0.66157106 \\
$\mathrm{C}$ & 3.24605263 & 4.24107632 & 0.39921670 \\
$\mathrm{C}$ & 2.00338123 & -0.66215390 & -1.32029905 \\
$\mathrm{H}$ & 3.08085224 & -0.84574860 & -1.33809164 \\
$\mathrm{H}$ & 1.51012006 & -1.63014790 & -1.43616846 \\
$\mathrm{H}$ & 1.74698320 & -0.04765279 & -2.19066218 \\
$\mathrm{C}$ & 2.12027560 & -0.83649343 & 1.16152915 \\
$\mathrm{H}$ & 1.73459367 & -0.54713213 & 2.13941191 \\
$\mathrm{H}$ & 1.87079511 & -1.88974963 & 1.01431325 \\
$\mathrm{H}$ & 3.21106434 & -0.75778138 & 1.19532875 \\
$\mathrm{O}$ & -0.45231610 & -1.37109980 & 0.08013698 \\
$\mathrm{C}$ & -1.64523853 & -1.65410423 & -0.49991776 \\
$\mathrm{O}$ & -2.33053314 & -0.83092136 & -1.05460038 \\
$\mathrm{C}$ & 4.10965029 & 4.20401828 & 1.68406970 \\
$\mathrm{H}$ & 5.14431615 & 4.49206974 & 1.48539697 \\
$\mathrm{H}$ & 3.73785869 & 4.83874935 & 2.49101078 \\
$\mathrm{H}$ & 4.15430629 & 3.19456422 & 2.08538235 \\
$\mathrm{C}$ & 3.32314332 & 5.70225062 & -0.28938200 \\
$\mathrm{C}$ & 1.09705956 & 4.99153423 & 1.59174491 \\
$\mathrm{H}$ & 0.03385527 & 4.78440599 & 1.73231224 \\
$\mathrm{H}$ & 1.54140454 & 4.98391333 & 2.59371049 \\
& & & \\
\hline
\end{tabular}




$\begin{array}{ccc}2.69568680 & 6.74138312 & 0.71417251 \\ 1.22815956 & 6.40451752 & 0.99991113 \\ 0.78626115 & 7.12290341 & 1.69638638 \\ 0.64190852 & 6.48451568 & 0.07694047 \\ 2.58212769 & 5.71678735 & -1.65441904 \\ 2.40495513 & 6.72784822 & -2.03019877 \\ 3.17357209 & 5.20933285 & -2.41902472 \\ 1.60827676 & 5.23191527 & -1.62665470 \\ 3.25104279 & 6.65997757 & 1.65351398 \\ 2.88777365 & 8.24088741 & 0.18400379 \\ 2.49617378 & 8.18662181 & -0.82941048 \\ 4.79466543 & 6.09790888 & -0.57597885 \\ 5.41658025 & 6.00529576 & 0.31622442 \\ 5.23360238 & 5.45475977 & -1.34178636 \\ 4.96433323 & 7.58254846 & -1.08780555 \\ 4.43893648 & 7.72300760 & -2.03241293 \\ 4.35734662 & 8.34835389 & 0.01406222 \\ 6.02387727 & 7.81623591 & -1.19806862 \\ 2.37868523 & 8.95376387 & 3.44570905 \\ 1.96264055 & 9.45704325 & 4.33075437 \\ 2.10725801 & 9.43822540 & 0.81634189 \\ 1.06188455 & 9.10579491 & 0.81913267 \\ 2.42589069 & 9.92447900 & 2.25305355 \\ 3.38270649 & 10.46083007 & 2.27162871 \\ 1.68039136 & 10.68932033 & 2.46864638 \\ 5.16839921 & 8.83987523 & 0.85835990 \\ 3.69364373 & 8.40729952 & 3.88948668 \\ 4.74280190 & 8.53586773 & 3.27800144 \\ 3.71164282 & 7.86523710 & 4.85013607 \\ 2.16969546 & 10.66876449 & -0.16336002 \\ 1.32406052 & 11.85736021 & 0.33135836 \\ 1.29905294 & 12.63706829 & -0.43412346 \\ 0.28747286 & 11.55395926 & 0.52137393 \\ 1.71705163 & 12.32059409 & 1.23837049 \\ -1.97525699 & -3.11746003 & -0.35449840 \\ -2.94961862 & -3.31493194 & -0.79901967 \\ -1.21111241 & -3.72448248 & -0.84869044 \\ -1.97998627 & -3.39886181 & 0.70215765 \\ 1.72607367 & 10.32911889 & -1.59985049 \\ 2.41816598 & 9.67284752 & -2.13494179 \\ 0.73449102 & 9.86139208 & -1.61211363 \\ 1.65848589 & 11.24552060 & -2.19139181 \\ 3.21787278 & 11.00318485 & -0.20367215 \\ 1.70310977 & 8.10020859 & 3.29692000 \\ 4.83243211 & 8.95763021 & 1.82708838\end{array}$

$V$ (Fig. 5S) $E=-\mathbf{1 5 5 2 . 4 4 5 8}$

$\begin{array}{lrrr}11 & & & \\ \mathrm{C} & 0.00000000 & 0.00000000 & 0.00000000 \\ \mathrm{C} & 1.54645791 & 0.00000000 & 0.00000000 \\ \mathrm{C} & 1.98572726 & 1.50661995 & 0.00000000 \\ \mathrm{C} & 1.39119044 & 2.43332591 & 1.11652447 \\ \mathrm{C} & -0.15502081 & 2.27685552 & 1.07492063 \\ \mathrm{C} & -0.62552460 & 0.81907472 & 1.12188796 \\ \mathrm{H} & -0.35164029 & 0.39933668 & -0.95744553 \\ \mathrm{H} & -0.61750669 & 2.81648424 & 1.90767404 \\ \mathrm{H} & -0.53899615 & 2.73642104 & 0.15371004 \\ \mathrm{H} & -0.39650243 & 0.35653306 & 2.08764326 \\ \mathrm{H} & -1.71294567 & 0.78752969 & 1.00998568 \\ \mathrm{H} & 1.54650464 & 1.89880200 & -0.93229068 \\ \mathrm{C} & 1.86324405 & 2.09106526 & 2.55203243 \\ \mathrm{H} & 2.87685505 & 1.69032973 & 2.59537472 \\ \mathrm{H} & 1.82590619 & 2.96788267 & 3.20316903\end{array}$




\begin{tabular}{|c|c|c|c|}
\hline & 1.21521135 & 1.34304806 & 3.01090635 \\
\hline $\mathrm{C}$ & 1.73566693 & 3.92013411 & 0.69403800 \\
\hline & 1.20641249 & 4.02168556 & -0.26166204 \\
\hline $\mathrm{C}$ & 3.49834922 & 1.72632051 & -0.13968177 \\
\hline & 3.91345837 & 1.04963367 & -0.89042244 \\
\hline & 4.02129209 & 1.49339581 & 0.79343625 \\
\hline $\mathrm{C}$ & 3.79377628 & 3.16349118 & -0.58830320 \\
\hline $\mathrm{H}$ & 3.34931573 & 3.28272063 & -1.58104411 \\
\hline $\mathrm{H}$ & 4.87560719 & 3.28980684 & -0.71373819 \\
\hline $\mathrm{C}$ & 3.24307758 & 4.24720018 & 0.37439366 \\
\hline $\mathrm{C}$ & 2.00264784 & -0.66085760 & -1.32127177 \\
\hline 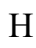 & 3.08054088 & -0.84173556 & -1.34124249 \\
\hline $\mathrm{H}$ & 1.51078713 & -1.62977749 & -1.43697099 \\
\hline $\mathrm{H}$ & 1.74330635 & -0.04625081 & -2.19067090 \\
\hline $\mathrm{C}$ & 2.12180910 & -0.83611291 & 1.16056782 \\
\hline $\mathrm{H}$ & 1.73540618 & -0.54788467 & 2.13843238 \\
\hline $\mathrm{H}$ & 1.87549323 & -1.89008643 & 1.01302405 \\
\hline $\mathrm{H}$ & 3.21240768 & -0.75349841 & 1.19369970 \\
\hline $\mathrm{O}$ & -0.45352514 & -1.37132776 & 0.08463346 \\
\hline $\mathrm{C}$ & -1.64085192 & -1.65828241 & -0.50325413 \\
\hline $\mathrm{O}$ & -2.32207574 & -0.84036510 & -1.07100658 \\
\hline $\mathrm{C}$ & 4.12895565 & 4.22688845 & 1.64584209 \\
\hline $\mathrm{H}$ & 5.18346651 & 4.36528962 & 1.39397382 \\
\hline $\mathrm{H}$ & 3.86201662 & 4.98545042 & 2.38529930 \\
\hline $\mathrm{H}$ & 4.06339370 & 3.26749127 & 2.15282585 \\
\hline $\mathrm{C}$ & 3.31896012 & 5.69587017 & -0.32133438 \\
\hline $\mathrm{C}$ & 1.12599958 & 4.99104831 & 1.61590215 \\
\hline $\mathrm{H}$ & 0.07501020 & 4.76902546 & 1.81347809 \\
\hline $\mathrm{H}$ & 1.62267171 & 5.00115961 & 2.59225793 \\
\hline $\mathrm{C}$ & 2.65668441 & 6.75711291 & 0.62102082 \\
\hline $\mathrm{C}$ & 1.21478441 & 6.38883965 & 0.99500505 \\
\hline $\mathrm{H}$ & 0.80988126 & 7.12472172 & 1.69909276 \\
\hline $\mathrm{H}$ & 0.57705946 & 6.43712615 & 0.10145042 \\
\hline $\mathrm{C}$ & 2.64855233 & 5.69067505 & -1.72069560 \\
\hline $\mathrm{H}$ & 2.48920523 & 6.69811051 & -2.12027410 \\
\hline $\mathrm{H}$ & 3.26864629 & 5.16921881 & -2.45323339 \\
\hline $\mathrm{H}$ & 1.66966923 & 5.21171192 & -1.72248715 \\
\hline $\mathrm{H}$ & 3.24156134 & 6.74371933 & 1.54223266 \\
\hline $\mathrm{C}$ & 2.71320529 & 8.20488564 & 0.05863853 \\
\hline $\mathrm{H}$ & 1.85494441 & 8.31899597 & -0.62118409 \\
\hline $\mathrm{C}$ & 4.79290599 & 6.14347879 & -0.50128055 \\
\hline $\mathrm{H}$ & 5.28484155 & 6.19992600 & 0.47137134 \\
\hline $\mathrm{H}$ & 5.35430650 & 5.41421793 & -1.08985212 \\
\hline $\mathrm{C}$ & 4.92281346 & 7.50706836 & -1.19436325 \\
\hline $\mathrm{H}$ & 4.93400863 & 7.42618638 & -2.28937743 \\
\hline $\mathrm{C}$ & 3.84152040 & 8.47119821 & -0.87898772 \\
\hline $\mathrm{H}$ & 5.87487125 & 8.00297477 & -0.95612222 \\
\hline $\mathrm{C}$ & 0.83187021 & 11.35453510 & 0.84147192 \\
\hline $\mathrm{H}$ & -0.22494611 & 11.62562414 & 0.96843999 \\
\hline $\mathrm{C}$ & 2.54385565 & 9.41333450 & 1.09447997 \\
\hline $\mathrm{H}$ & 3.11295018 & 10.25095062 & 0.67961838 \\
\hline $\mathrm{C}$ & 1.05384653 & 9.86756391 & 1.14783970 \\
\hline $\mathrm{H}$ & 0.45104624 & 9.29061274 & 0.44188844 \\
\hline $\mathrm{H}$ & 0.62600156 & 9.65763217 & 2.13126014 \\
\hline $\mathrm{O}$ & 3.87308023 & 9.56189257 & -1.53278355 \\
\hline $\mathrm{C}$ & 1.23424456 & 11.83339546 & -0.52157593 \\
\hline $\mathrm{O}$ & 1.95223522 & 11.25114574 & -1.31918538 \\
\hline $\mathrm{H}$ & 0.83550565 & 12.82159406 & -0.81291086 \\
\hline $\mathrm{C}$ & 3.18992897 & 9.17032836 & 2.48330688 \\
\hline $\mathrm{C}$ & 2.95045719 & 10.35978122 & 3.43124189 \\
\hline $\mathrm{H}$ & 3.44023198 & 10.17296917 & 4.39082478 \\
\hline $\mathrm{H}$ & 3.37911835 & 11.28341251 & 3.02397747 \\
\hline 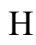 & 1.89255618 & 10.53467375 & 3.64120598 \\
\hline
\end{tabular}




$\begin{array}{lccc}\mathrm{C} & -1.97273418 & -3.12056072 & -0.34929817 \\ \mathrm{H} & -2.94439697 & -3.32064898 & -0.79863412 \\ \mathrm{H} & -1.20587127 & -3.73141810 & -0.83451957 \\ \mathrm{H} & -1.98417802 & -3.39460834 & 0.70922474 \\ \mathrm{C} & 4.70381301 & 8.94185083 & 2.36196783 \\ \mathrm{H} & 4.95947063 & 8.08484369 & 1.73248317 \\ \mathrm{H} & 5.19717350 & 9.82867104 & 1.94593161 \\ \mathrm{H} & 5.14658338 & 8.75619886 & 3.34432066 \\ \mathrm{H} & 2.72807513 & 8.28708835 & 2.94101836 \\ \mathrm{H} & 1.36305542 & 12.00748480 & 1.55307872 \\ \mathrm{H} & 3.07923006 & 10.18141973 & -1.36468195\end{array}$

\section{VI (Fig. 5S) E = -1552.4394}

11

$\mathrm{C} \quad 1.54680701$

$0.00000000 \quad 0.00000000$

$\begin{array}{lll}1.98496023 & 1.50693096 & 0.00000000\end{array}$

$\begin{array}{lll}1.39084640 & 2.43042236 & 1.12023535\end{array}$

$\begin{array}{lll}-0.15515663 & 2.27408749 & 1.08154621\end{array}$

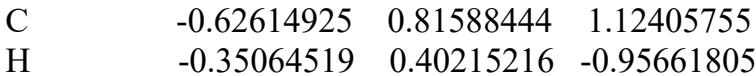

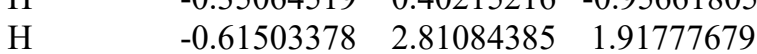

$\begin{array}{llll}\mathrm{H} & -0.54274395 & 2.73724618 & 0.16352070\end{array}$

$\begin{array}{llll}\mathrm{H} & -0.39679039 & 0.35065455 & 2.08823788\end{array}$

$\begin{array}{llll}\mathrm{H} & -1.71354295 & 0.78471447 & 1.01172936\end{array}$

$\begin{array}{llll}\mathrm{H} & 1.54169125 & 1.89963171 & -0.93015046\end{array}$

$\begin{array}{llll}\mathrm{C} & 1.86552712 & 2.08681842 & 2.55460051\end{array}$

$\begin{array}{llll}\mathrm{H} & 2.87992672 & 1.68735210 & 2.59677168\end{array}$

$\begin{array}{llll}\mathrm{H} & 1.82865676 & 2.96290026 & 3.20735465\end{array}$

$\begin{array}{llll}\mathrm{H} & 1.21885466 & 1.33789420 & 3.01365477\end{array}$

$\begin{array}{lllll}\mathrm{C} & 1.73306497 & 3.91618769 & 0.69581322\end{array}$

$\begin{array}{llll}\mathrm{H} & 1.21213260 & 4.00564805 & -0.26367989\end{array}$

$\begin{array}{lllll}\text { C } & 3.49758604 & 1.72847942 & -0.14424749\end{array}$

$\begin{array}{llll}\mathrm{H} & 3.90824017 & 1.05926066 & -0.90407851\end{array}$

$\begin{array}{llll}\mathrm{H} & 4.02410664 & 1.48209288 & 0.78327831\end{array}$

$\begin{array}{lllll}\mathrm{C} & 3.79993587 & 3.16887843 & -0.57882773\end{array}$

$\begin{array}{lllll}\mathrm{H} & 3.36845317 & 3.30028770 & -1.57581578\end{array}$

$\begin{array}{llll}\mathrm{H} & 4.88373307 & 3.29350872 & -0.68907374\end{array}$

$\begin{array}{llll}\text { C } & 3.24156480 & 4.24463852 & 0.38783522\end{array}$

$\begin{array}{llll}\mathrm{C} & 2.00364091 & -0.66154564 & -1.32072913\end{array}$

$\mathrm{H} \quad 3.08178744 \quad-0.84078755 \quad-1.33966359$

$\mathrm{H} \quad \begin{array}{llll}1.51382780 & -1.63153942 & -1.43520536\end{array}$

$\begin{array}{llll}\mathrm{H} & 1.74339761 & -0.04861232 & -2.19106573\end{array}$

$\begin{array}{llll}\mathrm{C} & 2.12137595 & -0.83705000 & 1.16046536\end{array}$

$\begin{array}{llll}\mathrm{H} & 1.73837071 & -0.54728138 & 2.13922219\end{array}$

$\begin{array}{llll}\mathrm{H} & 1.87072767 & -1.89013371 & 1.01354028\end{array}$

$\begin{array}{llll}\mathrm{H} & 3.21236743 & -0.75914065 & 1.19145949\end{array}$

$\begin{array}{llll}\mathrm{O} & -0.45305424 & -1.37112199 & 0.08193577\end{array}$

$\begin{array}{llll}\mathrm{C} & -1.63837382 & -1.65844172 & -0.51072353\end{array}$

$\begin{array}{lllll}\mathrm{O} & -2.31723573 & -0.84028506 & -1.08062479\end{array}$

$\begin{array}{lllll}\mathrm{C} & 4.11691110 & 4.21611297 & 1.66488299\end{array}$

$\begin{array}{lllll}\mathrm{H} & 5.16654474 & 4.41576675 & 1.43536561\end{array}$

$\begin{array}{llll}\mathrm{H} & 3.80373089 & 4.92269554 & 2.43662194\end{array}$

$\begin{array}{llll}\mathrm{H} & 4.09472422 & 3.23118699 & 2.12444824\end{array}$

$\begin{array}{llll}\mathrm{C} & 3.31140790 & 5.70251841 & -0.30688046\end{array}$

$\begin{array}{llll}\mathrm{C} & 1.10970594 & 4.99153605 & 1.60013459\end{array}$

$\begin{array}{llll}\mathrm{H} & 0.05224845 & 4.77749888 & 1.77043795\end{array}$

$\begin{array}{llll}\mathrm{H} & 1.57971245 & 4.99940881 & 2.58987619\end{array}$

$\begin{array}{llll}\mathrm{C} & 2.67000919 & 6.74954185 & 0.66792363\end{array}$

$\begin{array}{llll}\mathrm{C} & 1.21238281 & 6.39798117 & 0.99068990\end{array}$

$\begin{array}{llll}\mathrm{H} & 0.78676442 & 7.11386410 & 1.69865775\end{array}$

$\begin{array}{llll}\mathrm{H} & 0.60222303 & 6.46190638 & 0.08105737\end{array}$

$\begin{array}{lllll}\text { C } & 2.59358736 & 5.69687613 & -1.68409243\end{array}$ 


$\begin{array}{cccc} & & & \\ \mathrm{H} & 2.35278635 & 6.70250164 & -2.04009500 \\ \mathrm{H} & 3.22769839 & 5.24420171 & -2.44960588 \\ \mathrm{H} & 1.65307731 & 5.14870034 & -1.68009221 \\ \mathrm{H} & 3.23269675 & 6.70453879 & 1.60604384 \\ \mathrm{C} & 2.81323369 & 8.25405352 & 0.13941552 \\ \mathrm{H} & 2.37647430 & 8.23107113 & -0.86379576 \\ \mathrm{C} & 4.77944880 & 6.12217871 & -0.55398894 \\ \mathrm{H} & 5.36251442 & 6.08004248 & 0.36890250 \\ \mathrm{H} & 5.26812397 & 5.45719946 & -1.26968789 \\ \mathrm{C} & 4.90399989 & 7.57325331 & -1.11480059 \\ \mathrm{H} & 4.38289072 & 7.64944746 & -2.07253277 \\ \mathrm{C} & 4.26373694 & 8.46413981 & -0.11293219 \\ \mathrm{H} & 5.95044592 & 7.85044733 & -1.24868636 \\ \mathrm{C} & 3.97542256 & 9.40405702 & 2.76068516 \\ \mathrm{H} & 4.14272147 & 9.87479405 & 3.73444844 \\ \mathrm{C} & 2.06743190 & 9.35309289 & 0.93412062 \\ \mathrm{H} & 1.01750343 & 9.04284706 & 0.93929237 \\ \mathrm{C} & 2.47413467 & 9.41687276 & 2.42358728 \\ \mathrm{H} & 2.01299561 & 10.29834423 & 2.87141638 \\ \mathrm{H} & 2.02491855 & 8.56504339 & 2.94255432 \\ \mathrm{O} & 5.06720833 & 9.23291838 & 0.49326818 \\ \mathrm{C} & 4.86734210 & 10.13269262 & 1.79008949 \\ \mathrm{O} & 6.11700232 & 10.24871471 & 2.27841525 \\ \mathrm{H} & 4.45950920 & 11.04602624 & 1.35558436 \\ \mathrm{C} & 2.06037909 & 10.70770074 & 0.14008976 \\ \mathrm{C} & 1.60652253 & 11.90705421 & 0.98626753 \\ \mathrm{H} & 1.51863185 & 12.79606754 & 0.35573780 \\ \mathrm{H} & 0.62355461 & 11.72698837 & 1.43594679 \\ \mathrm{H} & 2.30120787 & 12.15297865 & 1.79478369 \\ \mathrm{C} & -1.97011406 & -3.12082170 & -0.35806196 \\ \mathrm{H} & -2.94028077 & -3.32131702 & -0.81038304 \\ \mathrm{H} & -1.20150442 & -3.73124617 & -0.84105173 \\ \mathrm{H} & -1.98464557 & -3.39495494 & 0.70038945 \\ \mathrm{C} & 1.15217620 & 10.60509518 & -1.09965817 \\ \mathrm{H} & 1.44277336 & 9.80499616 & -1.78667173 \\ \mathrm{H} & 0.11208948 & 10.42859005 & -0.80388421 \\ \mathrm{H} & 1.17943160 & 11.53785720 & -1.66950528 \\ \mathrm{H} & 3.07448014 & 10.92424868 & -0.22694582 \\ \mathrm{H} & 4.36969824 & 8.38663287 & 2.85604178 \\ \mathrm{H} & 6.63428532 & 10.88688548 & 1.76990815 \\ & & & \\ & & & \end{array}$

\section{VII (Fig. 6S) input structure}

11

C

C

C

C

C

C

$\mathrm{H}$

$\mathrm{H}$

$\mathrm{H}$

$\mathrm{H}$

$\mathrm{H}$

$\mathrm{H}$

C

$\mathrm{H}$

$\mathrm{H}$

$\mathrm{H}$

C

$\mathrm{H}$

C

$\mathrm{H}$
0.00000000
0.0000000
0.00000000
1.54720649
$\begin{array}{ll}0.00000000 & 0.00000000\end{array}$
1.98377350
1.50722698
0.00000000
1.38913102
2.42931875
1.12111756
$-0.15647352$
2.27224026
1.08480863
$-0.62583875$
0.81401746
1.12582413
$-0.35095345$
0.40354907
$-0.95589768$
$-0.61386822$
2.80680726
1.92354951
$-0.54538984$
2.73724041
0.16833514
$-0.39500580$
0.34806164
2.08941729
$-1.71320600$
0.78188349
1.01430165
1.53972302
1.89972985
1.86621141
2.09049087
$-0.93006175$
2.87843364
1.68564816
2.55617575
1.83609107
2.97094306
2.59833210
1.21655811
1.34696265
3.20282316
1.72928264
3.91477672
3.01989908
1.21815792
3.99704230
0.69370593
3.49622822
1.72989577
$-0.27230373$
3.90715535
1.06503277
$-0.14416566$
$-0.90788758$ 


\begin{tabular}{|c|c|c|c|}
\hline $\mathrm{H}$ & 4.02126811 & 1.47824643 & 0.78241691 \\
\hline $\mathrm{C}$ & 3.80325661 & 3.17327410 & -0.56868506 \\
\hline $\mathrm{H}$ & 3.37738441 & 3.31182348 & -1.56754128 \\
\hline $\mathrm{H}$ & 4.88816296 & 3.29501687 & -0.67108897 \\
\hline $\mathrm{C}$ & 3.24011134 & 4.24239000 & 0.40187259 \\
\hline $\mathrm{C}$ & 2.00518735 & -0.66215205 & -1.31980874 \\
\hline $\mathrm{H}$ & 3.08169776 & -0.85116806 & -1.33268735 \\
\hline $\mathrm{H}$ & 1.50722761 & -1.62742476 & -1.43937198 \\
\hline $\mathrm{H}$ & 1.75609670 & -0.04479065 & -2.19029937 \\
\hline $\mathrm{C}$ & 2.11952001 & -0.83569503 & 1.16268304 \\
\hline $\mathrm{H}$ & 1.73695669 & -0.53952488 & 2.13952606 \\
\hline $\mathrm{H}$ & 1.86443626 & -1.88817055 & 1.01959068 \\
\hline $\mathrm{H}$ & 3.21078726 & -0.76234102 & 1.19430944 \\
\hline $\mathrm{O}$ & -0.45426514 & -1.37117809 & 0.07914463 \\
\hline $\mathrm{C}$ & -1.64708209 & -1.65174225 & -0.50131031 \\
\hline $\mathrm{O}$ & -2.32876552 & -0.82880880 & -1.06110934 \\
\hline $\mathrm{C}$ & 4.10331122 & 4.21255762 & 1.68727870 \\
\hline $\mathrm{H}$ & 5.14947283 & 4.44578410 & 1.47342766 \\
\hline $\mathrm{H}$ & 3.76051816 & 4.89105293 & 2.47052829 \\
\hline $\mathrm{H}$ & 4.10702426 & 3.21614505 & 2.12185471 \\
\hline $\mathrm{C}$ & 3.31717955 & 5.70618112 & -0.28602638 \\
\hline $\mathrm{C}$ & 1.09439384 & 4.98921258 & 1.59025840 \\
\hline $\mathrm{H}$ & 0.03103034 & 4.78224353 & 1.73156971 \\
\hline $\mathrm{H}$ & 1.53991570 & 4.99870830 & 2.58936018 \\
\hline $\mathrm{C}$ & 2.68059530 & 6.74003889 & 0.69868346 \\
\hline $\mathrm{C}$ & 1.21455860 & 6.39936071 & 0.99058793 \\
\hline $\mathrm{H}$ & 0.78458781 & 7.10613923 & 1.70227526 \\
\hline $\mathrm{H}$ & 0.62160461 & 6.47645559 & 0.07031515 \\
\hline $\mathrm{C}$ & 2.60237735 & 5.71760084 & -1.66442611 \\
\hline $\mathrm{H}$ & 2.36709612 & 6.72916621 & -2.00976247 \\
\hline $\mathrm{H}$ & 3.23209199 & 5.26661148 & -2.43493756 \\
\hline $\mathrm{H}$ & 1.65821275 & 5.17686945 & -1.66466393 \\
\hline $\mathrm{H}$ & 3.22325430 & 6.69127161 & 1.64508877 \\
\hline $\mathrm{C}$ & 2.82792004 & 8.26696354 & 0.18816279 \\
\hline $\mathrm{H}$ & 2.29220268 & 8.26791147 & -0.76648697 \\
\hline $\mathrm{C}$ & 4.78655822 & 6.11298936 & -0.52632916 \\
\hline $\mathrm{H}$ & 5.36390576 & 6.09266149 & 0.40015437 \\
\hline $\mathrm{H}$ & 5.28308874 & 5.44160544 & -1.22999977 \\
\hline $\mathrm{C}$ & 4.90699267 & 7.55661632 & -1.12148227 \\
\hline $\mathrm{H}$ & 4.38724687 & 7.59595464 & -2.08297748 \\
\hline $\mathrm{C}$ & 4.24581217 & 8.45129221 & -0.16197466 \\
\hline $\mathrm{H}$ & 5.94941826 & 7.84808221 & -1.25803341 \\
\hline $\mathrm{C}$ & 4.44933698 & 9.73405578 & 2.41247371 \\
\hline $\mathrm{H}$ & 4.91260840 & 9.60053346 & 3.40042747 \\
\hline $\mathrm{C}$ & 2.20342200 & 9.36284133 & 1.10228681 \\
\hline $\mathrm{H}$ & 1.18572593 & 9.01533502 & 1.29868651 \\
\hline $\mathrm{C}$ & 2.92842550 & 9.51360123 & 2.47975163 \\
\hline $\mathrm{H}$ & 2.53080744 & 10.36661018 & 3.03979430 \\
\hline $\mathrm{H}$ & 2.75906194 & 8.60968106 & 3.08550057 \\
\hline $\mathrm{O}$ & 5.02235605 & 9.29354076 & 0.42564576 \\
\hline $\mathrm{C}$ & 4.84206905 & 11.12285251 & 1.95489689 \\
\hline $\mathrm{O}$ & 4.23988872 & 12.12935415 & 2.24532432 \\
\hline $\mathrm{H}$ & 5.75841867 & 11.16868085 & 1.33255676 \\
\hline $\mathrm{C}$ & 2.04618382 & 10.73486818 & 0.36808976 \\
\hline $\mathrm{C}$ & 1.28533366 & 11.74868670 & 1.23822525 \\
\hline $\mathrm{H}$ & 1.21224992 & 12.70764879 & 0.71856259 \\
\hline $\mathrm{H}$ & 0.26345171 & 11.40465773 & 1.43537031 \\
\hline $\mathrm{H}$ & 1.77091426 & 11.93896025 & 2.19859022 \\
\hline $\mathrm{C}$ & -1.98532863 & -3.11251373 & -0.34737195 \\
\hline $\mathrm{H}$ & -2.95219524 & -3.31061831 & -0.80777765 \\
\hline $\mathrm{H}$ & -1.21441559 & -3.72873688 & -0.81884067 \\
\hline $\mathrm{H}$ & -2.01262392 & -3.38139646 & 0.71232938 \\
\hline $\mathrm{C}$ & 1.34288802 & 10.61473224 & -0.99438352 \\
\hline
\end{tabular}




$\begin{array}{cccc}\mathrm{H} & 1.93693910 & 10.08617990 & -1.74558417 \\ \mathrm{H} & 0.37906551 & 10.10130775 & -0.89904757 \\ \mathrm{H} & 1.14248494 & 11.60985021 & -1.39995521 \\ \mathrm{H} & 3.04977272 & 11.15296430 & 0.18651479 \\ \mathrm{H} & 4.95336616 & 9.00400331 & 1.76800334 \\ \mathrm{H} & 4.84372884 & 12.87565706 & 2.24231142\end{array}$

VII (Fig. 6S) output structure, $E=\mathbf{- 1 5 5 2 . 4 3 0 7}$

\begin{tabular}{|c|c|c|c|}
\hline $\mathrm{C}$ & 0.00000000 & 0.00000000 & 0.00000000 \\
\hline $\mathrm{C}$ & 1.54721270 & 0.00000000 & 0.00000000 \\
\hline $\mathrm{C}$ & 1.98454475 & 1.50652849 & 0.00000000 \\
\hline $\mathrm{C}$ & 1.39282700 & 2.42843576 & 1.12291722 \\
\hline $\mathrm{C}$ & -0.15331389 & 2.27056608 & 1.08896801 \\
\hline $\mathrm{C}$ & -0.62340046 & 0.81247255 & 1.12796638 \\
\hline $\mathrm{H}$ & -0.35175796 & 0.40486695 & -0.95498631 \\
\hline $\mathrm{H}$ & -0.60992159 & 2.80418516 & 1.92883832 \\
\hline $\mathrm{H}$ & -0.54399142 & 2.73641299 & 0.17375162 \\
\hline $\mathrm{H}$ & -0.39188691 & 0.34359230 & 2.09012038 \\
\hline $\mathrm{H}$ & -1.71098173 & 0.78197875 & 1.01828018 \\
\hline $\mathrm{H}$ & 1.53992640 & 1.90045229 & -0.92892247 \\
\hline $\mathrm{C}$ & 1.87119975 & 2.08437479 & 2.55627267 \\
\hline $\mathrm{H}$ & 2.88550345 & 1.68496469 & 2.59714501 \\
\hline $\mathrm{H}$ & 1.83555549 & 2.96029897 & 3.20941465 \\
\hline $\mathrm{H}$ & 1.22572209 & 1.33461182 & 3.01595632 \\
\hline $\mathrm{C}$ & 1.73395387 & 3.91431273 & 0.69806001 \\
\hline $\mathrm{H}$ & 1.21584472 & 4.00145998 & -0.26335979 \\
\hline $\mathrm{C}$ & 3.49669993 & 1.72599338 & -0.14787403 \\
\hline $\mathrm{H}$ & 3.90404964 & 1.06026553 & -0.91261919 \\
\hline $\mathrm{H}$ & 4.02589131 & 1.47312314 & 0.77640904 \\
\hline $\mathrm{C}$ & 3.80279402 & 3.16797558 & -0.57576920 \\
\hline $\mathrm{H}$ & 3.37446153 & 3.30407133 & -1.57355782 \\
\hline $\mathrm{H}$ & 4.88724391 & 3.29092223 & -0.68195270 \\
\hline $\mathrm{C}$ & 3.24343158 & 4.24117474 & 0.39312489 \\
\hline $\mathrm{C}$ & 2.00578259 & -0.66372716 & -1.31870245 \\
\hline $\mathrm{H}$ & 3.08258763 & -0.85151733 & -1.33199090 \\
\hline $\mathrm{H}$ & 1.50914743 & -1.62995245 & -1.43579904 \\
\hline $\mathrm{H}$ & 1.75510399 & -0.04865295 & -2.19034877 \\
\hline $\mathrm{C}$ & 2.11921495 & -0.83464050 & 1.16376411 \\
\hline $\mathrm{H}$ & 1.72879179 & -0.54534683 & 2.13985057 \\
\hline $\mathrm{H}$ & 1.87354637 & -1.88870222 & 1.01612757 \\
\hline $\mathrm{H}$ & 3.20964048 & -0.75195904 & 1.20052108 \\
\hline $\mathrm{O}$ & -0.45344274 & -1.37131780 & 0.07798382 \\
\hline $\mathrm{C}$ & -1.64796674 & -1.65135973 & -0.49959123 \\
\hline $\mathrm{O}$ & -2.33242334 & -0.82714136 & -1.05390031 \\
\hline $\mathrm{C}$ & 4.11579996 & 4.20884315 & 1.67255897 \\
\hline $\mathrm{H}$ & 5.16171872 & 4.43697788 & 1.45297038 \\
\hline $\mathrm{H}$ & 3.78265441 & 4.89226721 & 2.45633685 \\
\hline $\mathrm{H}$ & 4.11603489 & 3.21543415 & 2.11442170 \\
\hline $\mathrm{C}$ & 3.31569441 & 5.70160470 & -0.29691813 \\
\hline $\mathrm{C}$ & 1.10613534 & 4.99090345 & 1.59900868 \\
\hline $\mathrm{H}$ & 0.04654249 & 4.77965868 & 1.75897388 \\
\hline $\mathrm{H}$ & 1.56621824 & 4.99569093 & 2.59335929 \\
\hline $\mathrm{C}$ & 2.68026969 & 6.74502456 & 0.68769946 \\
\hline $\mathrm{C}$ & 1.21744365 & 6.39925768 & 0.99395232 \\
\hline $\mathrm{H}$ & 0.78179166 & 7.11634874 & 1.69410976 \\
\hline $\mathrm{H}$ & 0.62078112 & 6.46448859 & 0.07585472 \\
\hline $\mathrm{C}$ & 2.59069739 & 5.70957341 & -1.67022161 \\
\hline $\mathrm{H}$ & 2.37897919 & 6.72061138 & -2.02853203 \\
\hline $\mathrm{H}$ & 3.20589384 & 5.23418771 & -2.43726387 \\
\hline $\mathrm{H}$ & 1.63438109 & 5.19038604 & -1.65692060 \\
\hline $\mathrm{H}$ & 3.23979720 & 6.69194714 & 1.62788837 \\
\hline $\mathrm{C}$ & 2.83732090 & 8.25229940 & 0.16933928 \\
\hline
\end{tabular}




$\begin{array}{lccc}\mathrm{H} & 2.37219741 & 8.24834481 & -0.82001056 \\ \mathrm{C} & 4.78568491 & 6.10947103 & -0.55455877 \\ \mathrm{H} & 5.37740094 & 6.06096993 & 0.36218756 \\ \mathrm{H} & 5.26340082 & 5.44571663 & -1.27856016 \\ \mathrm{C} & 4.92081636 & 7.56755128 & -1.11493685 \\ \mathrm{H} & 4.39889555 & 7.64917361 & -2.07072172 \\ \mathrm{C} & 4.28303479 & 8.41925133 & -0.09469058 \\ \mathrm{H} & 5.96974512 & 7.83905469 & -1.24157630 \\ \mathrm{C} & 3.79242448 & 9.57635385 & 3.01324482 \\ \mathrm{H} & 4.39876795 & 8.71593020 & 3.28649534 \\ \mathrm{C} & 2.11671409 & 9.36303716 & 0.99170877 \\ \mathrm{H} & 1.05445583 & 9.11589752 & 0.87909304 \\ \mathrm{C} & 2.37712132 & 9.33898923 & 2.52729189 \\ \mathrm{H} & 1.72066842 & 10.08647896 & 2.97829800 \\ \mathrm{H} & 2.04928616 & 8.37460619 & 2.92493602 \\ \mathrm{O} & 5.07209589 & 9.13353628 & 0.60566073 \\ \mathrm{C} & 4.29873083 & 10.76946674 & 3.38801308 \\ \mathrm{O} & 3.61033423 & 11.91448418 & 3.25152935 \\ \mathrm{H} & 5.29332703 & 10.84628718 & 3.82286230 \\ \mathrm{C} & 2.27899469 & 10.78773332 & 0.36757038 \\ \mathrm{C} & 1.20029506 & 11.74397976 & 0.90461268 \\ \mathrm{H} & 1.31637559 & 12.73270309 & 0.45167699 \\ \mathrm{H} & 0.19696667 & 11.38217950 & 0.65217494 \\ \mathrm{H} & 1.25696600 & 11.87785713 & 1.98581305 \\ \mathrm{C} & -1.98253299 & -3.11355013 & -0.35167071 \\ \mathrm{H} & -2.95796568 & -3.30842004 & -0.79507816 \\ \mathrm{H} & -1.22082688 & -3.72395856 & -0.84538954 \\ \mathrm{H} & -1.98762303 & -3.39304572 & 0.70550034 \\ \mathrm{C} & 2.22692181 & 10.79160692 & -1.16975075 \\ \mathrm{H} & 3.07302443 & 10.27621481 & -1.63768630 \\ \mathrm{H} & 1.30400544 & 10.33122918 & -1.54238559 \\ \mathrm{H} & 2.24843585 & 11.81987801 & -1.54004738 \\ \mathrm{H} & 3.25327344 & 11.19775861 & 0.66577571 \\ \mathrm{H} & 4.64217886 & 9.55300744 & 1.43258090 \\ \mathrm{H} & 4.12547170 & 12.66414407 & 3.57131913\end{array}$

\section{VIII (Fig. 7S) input structure}

$\begin{array}{lrrr}11 & & & \\ \mathrm{C} & 0.00000000 & 0.00000000 & 0.00000000 \\ \mathrm{C} & 1.54677895 & 0.00000000 & 0.00000000 \\ \mathrm{C} & 1.99018105 & 1.50473672 & 0.00000000 \\ \mathrm{C} & 1.38909759 & 2.43672037 & 1.10980561 \\ \mathrm{C} & -0.15629414 & 2.27588296 & 1.07188166 \\ \mathrm{C} & -0.62588801 & 0.81800239 & 1.12250864 \\ \mathrm{H} & -0.35218860 & 0.39902761 & -0.95724734 \\ \mathrm{H} & -0.61779349 & 2.81909067 & 1.90320543 \\ \mathrm{H} & -0.54186110 & 2.73325305 & 0.15052194 \\ \mathrm{H} & -0.39317850 & 0.35627132 & 2.08795766 \\ \mathrm{H} & -1.71340951 & 0.78379485 & 1.01227307 \\ \mathrm{H} & 1.56099624 & 1.89755035 & -0.93640310 \\ \mathrm{C} & 1.86389556 & 2.10118239 & 2.54613567 \\ \mathrm{H} & 2.89269300 & 1.74118326 & 2.59471454 \\ \mathrm{H} & 1.78791321 & 2.97323418 & 3.20153900 \\ \mathrm{H} & 1.24242414 & 1.32591745 & 2.99669211 \\ \mathrm{C} & 1.72976643 & 3.91904244 & 0.67498204 \\ \mathrm{H} & 1.20160604 & 4.01260195 & -0.28026584 \\ \mathrm{C} & 3.50633079 & 1.71937371 & -0.12574829 \\ \mathrm{H} & 3.92742608 & 1.03830313 & -0.86975158 \\ \mathrm{H} & 4.01779234 & 1.48281788 & 0.81338840 \\ \mathrm{C} & 3.81556617 & 3.15306456 & -0.57525889 \\ \mathrm{H} & 3.41128937 & 3.26401788 & -1.58533920 \\ \mathrm{H} & 4.90052283 & 3.28281101 & -0.66233077 \\ \mathrm{C} & 3.23229516 & 4.25128598 & 0.35058123\end{array}$




\begin{tabular}{|c|c|c|c|}
\hline $\mathrm{C}$ & 2.00368007 & -0.66521142 & -1.31853182 \\
\hline $\mathrm{H}$ & 3.08007127 & -0.85425677 & -1.33191373 \\
\hline $\mathrm{H}$ & 1.50510131 & -1.63049023 & -1.43621221 \\
\hline $\mathrm{H}$ & 1.75469229 & -0.04921173 & -2.18970423 \\
\hline $\mathrm{C}$ & 2.11647474 & -0.83675600 & 1.16332409 \\
\hline $\mathrm{H}$ & 1.72460226 & -0.54709554 & 2.13882930 \\
\hline $\mathrm{H}$ & 1.86947130 & -1.89068120 & 1.01574662 \\
\hline $\mathrm{H}$ & 3.20691583 & -0.75572148 & 1.20267722 \\
\hline $\mathrm{O}$ & -0.45148799 & -1.37311282 & 0.08558052 \\
\hline $\mathrm{C}$ & -1.64472377 & -1.66082522 & -0.48785097 \\
\hline $\mathrm{O}$ & -2.33615472 & -0.84382817 & -1.04445085 \\
\hline $\mathrm{C}$ & 4.09957284 & 4.25886012 & 1.63593444 \\
\hline $\mathrm{H}$ & 5.13892710 & 4.51385002 & 1.41699361 \\
\hline $\mathrm{H}$ & 3.74478402 & 4.95445811 & 2.40027874 \\
\hline $\mathrm{H}$ & 4.11842195 & 3.27273566 & 2.09570985 \\
\hline $\mathrm{C}$ & 3.28818098 & 5.68260655 & -0.38072800 \\
\hline $\mathrm{C}$ & 1.12770143 & 5.00069093 & 1.59141759 \\
\hline $\mathrm{H}$ & 0.06824023 & 4.79987943 & 1.77057278 \\
\hline $\mathrm{H}$ & 1.60511688 & 4.98294875 & 2.57891318 \\
\hline $\mathrm{C}$ & 2.69445905 & 6.76808340 & 0.57764796 \\
\hline $\mathrm{C}$ & 1.25602842 & 6.40569840 & 0.99149471 \\
\hline $\mathrm{H}$ & 0.86480374 & 7.13574203 & 1.71032012 \\
\hline $\mathrm{H}$ & 0.60109734 & 6.48510266 & 0.11569077 \\
\hline $\mathrm{C}$ & 2.51106798 & 5.69124669 & -1.72398511 \\
\hline $\mathrm{H}$ & 2.59012354 & 6.67137336 & -2.19936535 \\
\hline $\mathrm{H}$ & 2.92963297 & 4.97467465 & -2.43258484 \\
\hline $\mathrm{H}$ & 1.44953175 & 5.46616293 & -1.62185698 \\
\hline $\mathrm{H}$ & 3.31828085 & 6.75656595 & 1.48070817 \\
\hline $\mathrm{C}$ & 2.75736843 & 8.22124633 & 0.02348080 \\
\hline $\mathrm{H}$ & 1.91171728 & 8.35708363 & -0.66078510 \\
\hline $\mathrm{C}$ & 4.74975740 & 6.07746098 & -0.72377339 \\
\hline $\mathrm{H}$ & 5.42747866 & 5.86131004 & 0.10975584 \\
\hline $\mathrm{H}$ & 5.10891635 & 5.45810721 & -1.55328210 \\
\hline $\mathrm{C}$ & 4.89651876 & 7.52403074 & -1.10487910 \\
\hline $\mathrm{C}$ & 4.00436057 & 8.46669478 & -0.78871577 \\
\hline $\mathrm{H}$ & 5.76773366 & 7.80035569 & -1.69771434 \\
\hline $\mathrm{C}$ & 5.12291727 & 8.74699394 & 1.32076006 \\
\hline $\mathrm{H}$ & 6.00941118 & 8.81389545 & 1.98930904 \\
\hline $\mathrm{C}$ & 2.58079741 & 9.28994031 & 1.16871927 \\
\hline $\mathrm{H}$ & 1.97194187 & 8.81179865 & 1.94811664 \\
\hline $\mathrm{C}$ & 3.96322450 & 9.62181397 & 1.79984643 \\
\hline $\mathrm{H}$ & 4.21502570 & 10.66944574 & 1.60541956 \\
\hline $\mathrm{H}$ & 3.90385309 & 9.53023449 & 2.88594011 \\
\hline $\mathrm{O}$ & 4.11640690 & 9.77626419 & -1.18737587 \\
\hline $\mathrm{C}$ & 5.69932133 & 8.98273718 & 0.01359887 \\
\hline $\mathrm{O}$ & 5.97486147 & 10.16135229 & -0.38685062 \\
\hline $\mathrm{H}$ & 5.92769979 & 8.14696106 & -0.65375507 \\
\hline $\mathrm{C}$ & 1.77829879 & 10.53732230 & 0.68403299 \\
\hline $\mathrm{C}$ & 1.83265080 & 11.74663895 & 1.63165470 \\
\hline $\mathrm{H}$ & 1.26207878 & 12.57776266 & 1.20694555 \\
\hline $\mathrm{H}$ & 1.37176630 & 11.52487990 & 2.60466176 \\
\hline $\mathrm{H}$ & 2.84876814 & 12.12216438 & 1.79320910 \\
\hline $\mathrm{C}$ & -1.97080416 & -3.12500526 & -0.33451622 \\
\hline $\mathrm{H}$ & -2.95240966 & -3.32411210 & -0.76222739 \\
\hline $\mathrm{H}$ & -1.21506007 & -3.73160770 & -0.84209130 \\
\hline $\mathrm{H}$ & -1.95642596 & -3.40587907 & 0.72216481 \\
\hline $\mathrm{C}$ & 0.30262990 & 10.18122680 & 0.43833551 \\
\hline $\mathrm{H}$ & 0.17427549 & 9.35584919 & -0.26567308 \\
\hline $\mathrm{H}$ & -0.19411806 & 9.89905712 & 1.37487608 \\
\hline $\mathrm{H}$ & -0.23381576 & 11.04228402 & 0.02938971 \\
\hline $\mathrm{H}$ & 2.22248036 & 10.85087957 & -0.26550894 \\
\hline $\mathrm{H}$ & 4.91522643 & 7.66335727 & 1.36112679 \\
\hline $\mathrm{H}$ & 4.87189419 & 9.86223601 & -1.78273188 \\
\hline
\end{tabular}


VIII (Fig. 7S) output structure, $E=\mathbf{- 1 5 5 2 . 4 4 0 7}$

11

C

$\mathrm{C}$

$\begin{array}{lll}0.00000000 & 0.00000000 & 0.00000000\end{array}$

$\begin{array}{lll}1.54692122 & 0.00000000 & 0.00000000\end{array}$

C

1.98692614

$\begin{array}{ll}1.50621933 & 0.00000000\end{array}$

C

1.39214637

$2.43202759 \quad 1.11690389$

C

$-0.15391215$

$2.27863757 \quad 1.07330519$

$\mathrm{C}$

$-0.62649132$

$\begin{array}{ll}0.82138488 & 1.12014685\end{array}$

$\mathrm{H}$

$-0.35193473$

$-0.95859536$

$-0.61639877$

1.90528961

$\mathrm{H}$

$-0.53609427$

2.81938007

0.15171611

$-0.39918532$

.

2.08639898

$\mathrm{H}$

$-1.71366295$

$\begin{array}{lll}0.79119418 & 1.00655864\end{array}$

1.54635669

$1.89868334-0.93181777$

1.86097625

$\begin{array}{ll}2.08621076 & 2.55263370\end{array}$

$\begin{array}{lll}2.87857468 & 1.69526327 & 2.59910008\end{array}$

$\begin{array}{lll}1.81119201 & 2.95932516 & 3.20798060\end{array}$

$\begin{array}{lll}1.21913027 & 1.32881093 & 3.00447615\end{array}$

$\begin{array}{lll}1.73824962 & 3.92026230 & 0.69872306\end{array}$

$\begin{array}{lll}1.21154998 & 4.02348594 & -0.25824525\end{array}$

$\begin{array}{llll}3.49981044 & 1.72679580 & -0.14179527\end{array}$

$\begin{array}{llll}3.91265819 & 1.05177749 & -0.89532897\end{array}$

$\begin{array}{llll}4.02501685 & 1.49098097 & 0.78929374\end{array}$

$\begin{array}{llll}3.79326679 & 3.16542844 & -0.58809688\end{array}$

$\begin{array}{llll}3.34134702 & 3.28667066 & -1.57734618\end{array}$

$\begin{array}{llll}4.87458632 & 3.29314340 & -0.72090360\end{array}$

$\begin{array}{lll}3.24757538 & 4.24408915 & 0.38223913\end{array}$

$\begin{array}{llll}2.00487416 & -0.66246317 & -1.31981571\end{array}$

$\begin{array}{llll}3.08167141 & -0.85098186 & -1.33375713\end{array}$

$\begin{array}{llll}1.50726637 & -1.62808998 & -1.43867733\end{array}$

$\begin{array}{lll}1.75478376 & -0.04520925 & -2.19012188\end{array}$

$\begin{array}{lll}2.11796907 & -0.83732963 & 1.16258004\end{array}$

$\begin{array}{lll}1.72439410 & -0.55151090 & 2.13818505\end{array}$

$\begin{array}{llll}1.87336819 & -1.89113891 & 1.01127353\end{array}$

$\begin{array}{lll}3.20826568 & -0.75459331 & 1.20371212\end{array}$

$\begin{array}{lll}-0.45196195 & -1.37123245 & 0.09000845\end{array}$

$\begin{array}{llll}-1.64327075 & -1.65995761 & -0.48966721\end{array}$

$\begin{array}{llll}-2.32454702 & -0.84469567 & -1.06084705\end{array}$

$\begin{array}{lll}4.13059659 & 4.21139410 & 1.65439370\end{array}$

$\begin{array}{lll}5.18878643 & 4.32305985 & 1.40309594\end{array}$

$\begin{array}{lll}3.87868855 & 4.98019472 & 2.38851089\end{array}$

$\begin{array}{lll}4.04195258 & 3.25674231 & 2.16523158\end{array}$

$\begin{array}{lll}3.32596374 & 5.69807553 & -0.30010946\end{array}$

$\begin{array}{lll}1.12864058 & 4.98935721 & 1.62273758\end{array}$

$\begin{array}{lll}0.07851870 & 4.76519977 & 1.82125387\end{array}$

$\begin{array}{llll}1.62661091 & 4.99952230 & 2.59849935\end{array}$

$\begin{array}{lll}2.65591508 & 6.75466276 & 0.63362498\end{array}$

$\begin{array}{lll}1.21454727 & 6.38793289 & 1.00268248\end{array}$

$\begin{array}{lll}0.81248464 & 7.12862234 & 1.70239527\end{array}$

$\begin{array}{llll}0.58046116 & 6.43623987 & 0.10715881\end{array}$

$\begin{array}{llll}2.66970770 & 5.70915086 & -1.70337989\end{array}$

$\begin{array}{llll}2.52042364 & 6.72508997 & -2.09230932\end{array}$

$\begin{array}{llll}3.28805622 & 5.19092660 & -2.43936257\end{array}$

$\begin{array}{llll}1.68243413 & 5.24783977 & -1.71633191\end{array}$

$\begin{array}{lll}3.23626185 & 6.75264300 & 1.56182832\end{array}$

$\begin{array}{llll}2.72657659 & 8.21004301 & 0.07306992\end{array}$

$\begin{array}{llll}1.75899992 & 8.51476562 & -0.33248403\end{array}$

$\begin{array}{llll}4.79825819 & 6.15643310 & -0.46095240\end{array}$

$\begin{array}{llll}5.28523642 & 6.16643067 & 0.51465554\end{array}$

$\begin{array}{lllll}\mathrm{H} & & 5.36053949 & 5.45615962 & -1.08341422 \\ \mathrm{C} & & 4.92933799 & 7.55630800 & -1.09816784\end{array}$ 


$\begin{array}{lrcc}\mathrm{C} & 3.71167792 & 8.38742023 & -0.98758801 \\ \mathrm{H} & 5.18105561 & 7.47933056 & -2.16109719 \\ \mathrm{C} & 5.75692504 & 8.90532873 & 0.97248687 \\ \mathrm{H} & 6.65595172 & 9.42342637 & 1.32015227 \\ \mathrm{C} & 3.10823483 & 9.32616324 & 1.18826572 \\ \mathrm{H} & 2.98219575 & 8.78558534 & 2.13339754 \\ \mathrm{C} & 4.56489948 & 9.87131848 & 1.14994765 \\ \mathrm{H} & 4.64424215 & 10.66054560 & 0.39421521 \\ \mathrm{H} & 4.71898573 & 10.37396102 & 2.10616023 \\ \mathrm{O} & 3.59358510 & 9.42185025 & -1.74559796 \\ \mathrm{C} & 6.05507235 & 8.45051190 & -0.46209044 \\ \mathrm{O} & 6.20635285 & 9.60303109 & -1.30699320 \\ \mathrm{H} & 6.96891257 & 7.84881587 & -0.47084727 \\ \mathrm{C} & 2.08770613 & 10.50194459 & 1.15137439 \\ \mathrm{C} & 2.45509137 & 11.62085698 & 2.14441672 \\ \mathrm{H} & 1.66790861 & 12.37951563 & 2.14680942 \\ \mathrm{H} & 2.53646208 & 11.23335902 & 3.16677253 \\ \mathrm{H} & 3.38924831 & 12.12680134 & 1.89430258 \\ \mathrm{C} & -1.97894283 & -3.11968622 & -0.32049890 \\ \mathrm{H} & -2.95367973 & -3.32063649 & -0.76269467 \\ \mathrm{H} & -1.21692576 & -3.73805851 & -0.80380984 \\ \mathrm{H} & -1.98583001 & -3.38332468 & 0.74071029 \\ \mathrm{C} & 0.64708748 & 10.05093407 & 1.43831513 \\ \mathrm{H} & 0.26477047 & 9.30882483 & 0.73209401 \\ \mathrm{H} & 0.56232408 & 9.62837977 & 2.44607577 \\ \mathrm{H} & -0.02738893 & 10.90982809 & 1.38680473 \\ \mathrm{H} & 2.12243423 & 10.92947246 & 0.13903602 \\ \mathrm{H} & 5.65017304 & 8.02404401 & 1.61393417 \\ \mathrm{H} & 4.46542705 & 9.61689708 & -2.16807831 \\ \mathrm{H} & 7.01741442 & 9.52681622 & -1.82188754\end{array}$

\section{IX (Fig. 7S) input structure}

$\begin{array}{lrrr}11 & & & \\ \mathrm{C} & 0.00000000 & 0.00000000 & 0.00000000 \\ \mathrm{C} & 1.54677919 & 0.00000000 & 0.00000000 \\ \mathrm{C} & 1.99018096 & 1.50473706 & 0.00000000 \\ \mathrm{C} & 1.38909712 & 2.43672081 & 1.10980516 \\ \mathrm{C} & -0.15629440 & 2.27588345 & 1.07188097 \\ \mathrm{C} & -0.62588813 & 0.81800290 & 1.12250917 \\ \mathrm{H} & -0.35218870 & 0.39902685 & -0.95724757 \\ \mathrm{H} & -0.61779406 & 2.81909129 & 1.90320495 \\ \mathrm{H} & -0.54186082 & 2.73325305 & 0.15052140 \\ \mathrm{H} & -0.39317832 & 0.35627150 & 2.08795800 \\ \mathrm{H} & -1.71340917 & 0.78379423 & 1.01227347 \\ \mathrm{H} & 1.56099625 & 1.89755018 & -0.93640354 \\ \mathrm{C} & 1.86389585 & 2.10118351 & 2.54613500 \\ \mathrm{H} & 2.89269282 & 1.74118420 & 2.59471426 \\ \mathrm{H} & 1.78791262 & 2.97323575 & 3.20153851 \\ \mathrm{H} & 1.24242382 & 1.32591821 & 2.99669231 \\ \mathrm{C} & 1.72976599 & 3.91904326 & 0.67498061 \\ \mathrm{H} & 1.20160509 & 4.01260188 & -0.28026669 \\ \mathrm{C} & 3.50633020 & 1.71937402 & -0.12574871 \\ \mathrm{H} & 3.92742601 & 1.03830328 & -0.86975177 \\ \mathrm{H} & 4.01779243 & 1.48281816 & 0.81338792 \\ \mathrm{C} & 3.81556607 & 3.15306489 & -0.57525940 \\ \mathrm{H} & 3.41128878 & 3.26401826 & -1.58534000 \\ \mathrm{H} & 4.90052274 & 3.28281137 & -0.66233208 \\ \mathrm{C} & 3.23229503 & 4.25128631 & 0.35057993 \\ \mathrm{C} & 2.00368013 & -0.66521115 & -1.31853102 \\ \mathrm{H} & 3.08007149 & -0.85425687 & -1.33191277 \\ \mathrm{H} & 1.50510131 & -1.63049039 & -1.43621120 \\ \mathrm{H} & 1.75469220 & -0.04921244 & -2.18970429 \\ \mathrm{C} & 2.11647527 & -0.83675583 & 1.16332505\end{array}$




\begin{tabular}{|c|c|c|c|}
\hline $\mathrm{H}$ & 1.72460299 & -0.54709433 & 2.13883021 \\
\hline $\mathrm{H}$ & 1.86947164 & -1.89068034 & 1.01574804 \\
\hline $\mathrm{H}$ & 3.20691650 & -0.75572026 & 1.20267721 \\
\hline $\mathrm{O}$ & -0.45148721 & -1.37311305 & 0.08558127 \\
\hline $\mathrm{C}$ & -1.64472385 & -1.66082615 & -0.48785024 \\
\hline $\mathrm{O}$ & -2.33615423 & -0.84382869 & -1.04445022 \\
\hline $\mathrm{C}$ & 4.09957228 & 4.25886168 & 1.63593327 \\
\hline $\mathrm{H}$ & 5.13892669 & 4.51385128 & 1.41699216 \\
\hline $\mathrm{H}$ & 3.74478352 & 4.95445907 & 2.40027668 \\
\hline $\mathrm{H}$ & 4.11842128 & 3.27273684 & 2.09570868 \\
\hline $\mathrm{C}$ & 3.28817996 & 5.68260643 & -0.38072956 \\
\hline $\mathrm{C}$ & 1.12770095 & 5.00069164 & 1.59141595 \\
\hline $\mathrm{H}$ & 0.06823950 & 4.79987971 & 1.77057159 \\
\hline $\mathrm{H}$ & 1.60511646 & 4.98295000 & 2.57891135 \\
\hline $\mathrm{C}$ & 2.69445756 & 6.76808390 & 0.57764578 \\
\hline $\mathrm{C}$ & 1.25602769 & 6.40569902 & 0.99149263 \\
\hline $\mathrm{H}$ & 0.86480250 & 7.13574310 & 1.71031796 \\
\hline $\mathrm{H}$ & 0.60109609 & 6.48510244 & 0.11568925 \\
\hline $\mathrm{C}$ & 2.51106745 & 5.69124608 & -1.72398666 \\
\hline $\mathrm{H}$ & 2.59012261 & 6.67137298 & -2.19936726 \\
\hline $\mathrm{H}$ & 2.92963252 & 4.97467378 & -2.43258608 \\
\hline $\mathrm{H}$ & 1.44953036 & 5.46616223 & -1.62185885 \\
\hline $\mathrm{H}$ & 3.31828033 & 6.75656688 & 1.48070597 \\
\hline $\mathrm{C}$ & 2.75736695 & 8.22124722 & 0.02347788 \\
\hline $\mathrm{H}$ & 1.91171557 & 8.35708317 & -0.66078784 \\
\hline $\mathrm{C}$ & 4.74975692 & 6.07746153 & -0.72377537 \\
\hline $\mathrm{H}$ & 5.42747786 & 5.86131131 & 0.10975420 \\
\hline $\mathrm{H}$ & 5.10891545 & 5.45810729 & -1.55328374 \\
\hline $\mathrm{C}$ & 4.89651716 & 7.52403101 & -1.10488207 \\
\hline $\mathrm{C}$ & 4.00435911 & 8.46669504 & -0.78871850 \\
\hline $\mathrm{H}$ & 5.76773240 & 7.80035548 & -1.69771698 \\
\hline $\mathrm{C}$ & 5.12291640 & 8.74699515 & 1.32075675 \\
\hline $\mathrm{H}$ & 6.00940963 & 8.81389700 & 1.98930568 \\
\hline $\mathrm{C}$ & 2.58079599 & 9.28994117 & 1.16871580 \\
\hline $\mathrm{H}$ & 1.97194035 & 8.81180005 & 1.94811407 \\
\hline $\mathrm{C}$ & 3.96322284 & 9.62181520 & 1.79984323 \\
\hline $\mathrm{H}$ & 4.21502396 & 10.66944710 & 1.60541583 \\
\hline $\mathrm{H}$ & 3.90385219 & 9.53023642 & 2.88593653 \\
\hline $\mathrm{O}$ & 4.11640502 & 9.77626399 & -1.18737898 \\
\hline $\mathrm{C}$ & 5.69931934 & 8.98273784 & 0.01359552 \\
\hline $\mathrm{O}$ & 6.41393537 & 8.09962378 & -0.56506734 \\
\hline $\mathrm{H}$ & 5.51709158 & 9.92290509 & -0.51453169 \\
\hline $\mathrm{C}$ & 1.77829748 & 10.53732297 & 0.68402903 \\
\hline $\mathrm{C}$ & 1.83264860 & 11.74663942 & 1.63165037 \\
\hline $\mathrm{H}$ & 1.26207701 & 12.57776339 & 1.20694079 \\
\hline $\mathrm{H}$ & 1.37176457 & 11.52488090 & 2.60465732 \\
\hline $\mathrm{H}$ & 2.84876582 & 12.12216533 & 1.79320467 \\
\hline $\mathrm{C}$ & -1.97080409 & -3.12500619 & -0.33451490 \\
\hline $\mathrm{H}$ & -2.95240923 & -3.32411306 & -0.76222552 \\
\hline $\mathrm{H}$ & -1.21505900 & -3.73160835 & -0.84208906 \\
\hline $\mathrm{H}$ & -1.95642463 & -3.40587955 & 0.72216688 \\
\hline $\mathrm{C}$ & 0.30262799 & 10.18122649 & 0.43833182 \\
\hline $\mathrm{H}$ & 0.17427428 & 9.35584850 & -0.26567591 \\
\hline $\mathrm{H}$ & -0.19411985 & 9.89905749 & 1.37487255 \\
\hline $\mathrm{H}$ & -0.23381773 & 11.04228431 & 0.02938589 \\
\hline $\mathrm{H}$ & 2.22247849 & 10.85088013 & -0.26551289 \\
\hline $\mathrm{H}$ & 4.91522532 & 7.66335824 & 1.36112456 \\
\hline $\mathrm{H}$ & 4.87189268 & 9.86223631 & -1.78273538 \\
\hline $\mathrm{H}$ & 6.73517835 & 8.35830475 & -1.44910854 \\
\hline
\end{tabular}

IX (Fig. 7S) output structure, $E=\mathbf{- 1 5 5 2 . 4 3 1 9}$

11

$\begin{array}{llll}\text { C } & 0.00000000 & 0.00000000 & 0.00000000\end{array}$ 


\begin{tabular}{|c|c|c|c|}
\hline $\mathrm{C}$ & 84660 & 00000 & 000 \\
\hline $\mathrm{C}$ & 1.98413832 & 1.50725901 & 0.00000000 \\
\hline $\mathrm{C}$ & 1.39160640 & 2.42979502 & 1.12110066 \\
\hline $\mathrm{C}$ & -0.15434737 & 2.27653448 & 1.07947604 \\
\hline $\mathrm{C}$ & -0.62755663 & 0.81911982 & 1.12109736 \\
\hline $\mathrm{H}$ & -0.35114676 & 0.39813756 & -0.95809134 \\
\hline $\mathrm{H}$ & -0.61581718 & 2.81427981 & 1.91398111 \\
\hline $\mathrm{H}$ & -0.53740452 & 2.74022595 & 0.16006762 \\
\hline $\mathrm{H}$ & -0.40352271 & 0.35379459 & 2.08673395 \\
\hline $\mathrm{H}$ & -1.71462484 & 0.78939207 & 1.00468157 \\
\hline $\mathrm{H}$ & 1.54025403 & 1.89990483 & -0.92999311 \\
\hline $\mathrm{C}$ & 1.86302826 & 2.07814105 & 2.55464794 \\
\hline $\mathrm{H}$ & 2.87511098 & 1.67266753 & 2.59720454 \\
\hline $\mathrm{H}$ & 1.82782484 & 2.95085047 & 3.21142741 \\
\hline $\mathrm{H}$ & 1.21244361 & 1.32984130 & 3.00896889 \\
\hline $\mathrm{C}$ & 1.73373579 & 3.92070807 & 0.70845421 \\
\hline $\mathrm{H}$ & 1.19683859 & 4.03291232 & -0.24213640 \\
\hline $\mathrm{C}$ & 3.49603462 & 1.73227614 & -0.14670384 \\
\hline $\mathrm{H}$ & 3.90944722 & 1.05876593 & -0.90143269 \\
\hline $\mathrm{H}$ & 4.02557903 & 1.49941336 & 0.78272161 \\
\hline $\mathrm{C}$ & 3.78179579 & 3.17119468 & -0.59474850 \\
\hline $\mathrm{H}$ & 3.32344371 & 3.29064177 & -1.58140742 \\
\hline $\mathrm{H}$ & 4.86147490 & 3.30432332 & -0.73328836 \\
\hline $\mathrm{C}$ & 3.23888949 & 4.24805010 & 0.37919601 \\
\hline $\mathrm{C}$ & 2.00384471 & -0.66153740 & -1.32044450 \\
\hline $\mathrm{H}$ & 3.08034788 & -0.85103768 & -1.33456695 \\
\hline $\mathrm{H}$ & 1.50561872 & -1.62672145 & -1.43971744 \\
\hline $\mathrm{H}$ & 1.75369902 & -0.04402037 & -2.19054960 \\
\hline $\mathrm{C}$ & 2.12191326 & -0.83564244 & 1.16095839 \\
\hline $\mathrm{H}$ & 1.72683851 & -0.55352015 & 2.13694902 \\
\hline $\mathrm{H}$ & 1.88439030 & -1.89082151 & 1.00831449 \\
\hline $\mathrm{H}$ & 3.21173272 & -0.74466801 & 1.20082753 \\
\hline $\mathrm{O}$ & -0.45273859 & -1.37126355 & 0.08715885 \\
\hline $\mathrm{C}$ & -1.63869745 & -1.66106846 & -0.50278309 \\
\hline $\mathrm{O}$ & -2.31679788 & -0.84617221 & -1.07832298 \\
\hline $\mathrm{C}$ & 4.12998214 & 4.21475522 & 1.64715034 \\
\hline $\mathrm{H}$ & 5.19049122 & 4.28201856 & 1.39106755 \\
\hline $\mathrm{H}$ & 3.91097129 & 5.00875954 & 2.36432456 \\
\hline $\mathrm{H}$ & 4.01025045 & 3.27730448 & 2.18337357 \\
\hline $\mathrm{C}$ & 3.30900760 & 5.70487434 & -0.30530375 \\
\hline $\mathrm{C}$ & 1.13586848 & 4.98328058 & 1.64885418 \\
\hline $\mathrm{H}$ & 0.08758123 & 4.76012728 & 1.85857301 \\
\hline $\mathrm{H}$ & 1.64583460 & 4.98556743 & 2.61822019 \\
\hline $\mathrm{C}$ & 2.65055131 & 6.75330259 & 0.64312323 \\
\hline $\mathrm{C}$ & 1.21572446 & 6.38494369 & 1.03550524 \\
\hline $\mathrm{H}$ & 0.82414635 & 7.12406768 & 1.74381996 \\
\hline $\mathrm{H}$ & 0.56661245 & 6.43772378 & 0.15128261 \\
\hline $\mathrm{C}$ & 2.62612313 & 5.72270837 & -1.69524894 \\
\hline $\mathrm{H}$ & 2.54224795 & 6.73668029 & -2.10790361 \\
\hline $\mathrm{H}$ & 3.19580067 & 5.14836040 & -2.42782567 \\
\hline $\mathrm{H}$ & 1.60978562 & 5.32878334 & -1.68150650 \\
\hline $\mathrm{H}$ & 3.24577028 & 6.76347515 & 1.56053543 \\
\hline $\mathrm{C}$ & 2.68166176 & 8.20778546 & 0.07261189 \\
\hline $\mathrm{H}$ & 1.73176744 & 8.42976029 & -0.41957776 \\
\hline $\mathrm{C}$ & 4.78053896 & 6.14612452 & -0.49490822 \\
\hline $\mathrm{H}$ & 5.34240504 & 6.02597456 & 0.42844403 \\
\hline $\mathrm{H}$ & 5.27439568 & 5.51381546 & -1.23621357 \\
\hline $\mathrm{C}$ & 4.96034495 & 7.62199837 & -0.96163766 \\
\hline $\mathrm{C}$ & 3.71536065 & 8.38684764 & -0.95111324 \\
\hline $\mathrm{H}$ & 5.39380283 & 7.66855129 & -1.96894657 \\
\hline $\mathrm{C}$ & 5.46523876 & 8.76946797 & 1.36902034 \\
\hline $\mathrm{H}$ & 6.32245544 & 9.17705885 & 1.91267441 \\
\hline $\mathrm{C}$ & 2.87313898 & 9.32593151 & 1.20557987 \\
\hline
\end{tabular}




$\begin{array}{lccc}\mathrm{H} & 2.58691684 & 8.81049301 & 2.13021698 \\ \mathrm{C} & 4.33321357 & 9.81480902 & 1.37232716 \\ \mathrm{H} & 4.55081027 & 10.57943218 & 0.61241852 \\ \mathrm{H} & 4.38176415 & 10.34387236 & 2.32550493 \\ \mathrm{O} & 3.50136625 & 9.33092499 & -1.81413966 \\ \mathrm{C} & 6.00756109 & 8.41357680 & -0.01378779 \\ \mathrm{O} & 7.13906771 & 7.61549732 & 0.16648468 \\ \mathrm{H} & 6.25838022 & 9.34303854 & -0.54594661 \\ \mathrm{C} & 1.89137256 & 10.51411810 & 0.99793602 \\ \mathrm{C} & 2.13554123 & 11.64702285 & 2.01185288 \\ \mathrm{H} & 1.37419877 & 12.42158122 & 1.88693177 \\ \mathrm{H} & 2.06044589 & 11.27979053 & 3.04209775 \\ \mathrm{H} & 3.10845314 & 12.12726545 & 1.89050373 \\ \mathrm{C} & -1.97298604 & -3.12170181 & -0.33867549 \\ \mathrm{H} & -2.94352472 & -3.32399497 & -0.78940989 \\ \mathrm{H} & -1.20564865 & -3.73788870 & -0.81635788 \\ \mathrm{H} & -1.98831231 & -3.38712826 & 0.72200005 \\ \mathrm{C} & 0.41851161 & 10.08426920 & 1.08800487 \\ \mathrm{H} & 0.13459176 & 9.31744810 & 0.36139659 \\ \mathrm{H} & 0.18539173 & 9.69832263 & 2.08689887 \\ \mathrm{H} & -0.23279183 & 10.94405717 & 0.90952261 \\ \mathrm{H} & 2.07283097 & 10.92133423 & -0.00754396 \\ \mathrm{H} & 5.18112016 & 7.85900772 & 1.90621199 \\ \mathrm{H} & 4.22068709 & 9.41122338 & -2.46560607 \\ \mathrm{H} & 7.66993918 & 7.59407267 & -0.63905355\end{array}$

\section{X (Fig. 8S), E = -1552.4359}

11

C

C

C

C

$\mathrm{C}$

$\mathrm{H}$

$\mathrm{H}$

$\mathrm{H}$

$\mathrm{H}$

$\mathrm{H}$

$\mathrm{H}$

C

$\mathrm{H}$

$\mathrm{H}$

$\mathrm{H}$

C

$\mathrm{H}$

C

$\mathrm{H}$

$\mathrm{H}$

C

$\mathrm{H}$

$\mathrm{H}$

C

C

$\mathrm{H}$

$\mathrm{H}$

$\mathrm{H}$

C

$\mathrm{H}$

$\mathrm{H}$

$\mathrm{H}$

$\mathrm{O}$

C

$\begin{array}{rrr}0.00000000 & 0.00000000 & 0.00000000 \\ 1.54673259 & 0.00000000 & 0.00000000 \\ 1.98550759 & 1.50708823 & 0.00000000 \\ 1.39141921 & 2.43060888 & 1.11977499 \\ -0.15413609 & 2.27565861 & 1.07923394 \\ -0.62568147 & 0.81783406 & 1.12255158 \\ -0.35106994 & 0.39846893 & -0.95781373 \\ -0.61497020 & 2.81353204 & 1.91397515 \\ -0.53946814 & 2.73813099 & 0.16017605 \\ -0.39607212 & 0.35282910 & 2.08705921 \\ -1.71315011 & 0.78617770 & 1.01044174 \\ 1.54306141 & 1.90028094 & -0.93032180 \\ 1.86276380 & 2.08468734 & 2.55504443 \\ 2.87534483 & 1.68120411 & 2.59806732 \\ 1.82855415 & 2.96195778 & 3.20598269 \\ 1.21267467 & 1.33840314 & 3.01387201 \\ 1.73754571 & 3.91775049 & 0.70138885 \\ 1.22184221 & 4.01449601 & -0.26149942 \\ 3.49810830 & 1.72844728 & -0.14353892 \\ 3.90859363 & 1.06130829 & -0.90565338 \\ 4.02326848 & 1.47835018 & 0.78400912 \\ 3.80086117 & 3.17126987 & -0.57132750 \\ 3.36474863 & 3.30898277 & -1.56567855 \\ 4.88437953 & 3.29366949 & -0.68744968 \\ 3.24867992 & 4.24523198 & 0.40092363 \\ 2.00301153 & -0.66275428 & -1.32010037 \\ 3.07960359 & -0.85137283 & -1.33534368 \\ 1.50502874 & -1.62826468 & -1.43861879 \\ 1.75164815 & -0.04531600 & -2.18992010 \\ 2.11993220 & -0.83604200 & 1.16228372 \\ 1.73091355 & -0.54783077 & 2.13922982 \\ 1.87488998 & -1.89024966 & 1.01421715 \\ 3.21035021 & -0.75251506 & 1.19801766 \\ -0.45283086 & -1.37187152 & 0.08638243 \\ -1.64009187 & -1.66113300 & -0.49973341\end{array}$




\begin{tabular}{|c|c|c|c|}
\hline $\mathrm{O}$ & -2.32111613 & -0.84652953 & -1.07240303 \\
\hline $\mathrm{C}$ & 4.11912652 & 4.20033514 & 1.68090660 \\
\hline $\mathrm{H}$ & 5.17348300 & 4.37299548 & 1.44989858 \\
\hline $\mathrm{H}$ & 3.82096367 & 4.92284502 & 2.44391456 \\
\hline $\mathrm{H}$ & 4.07305564 & 3.22150386 & 2.15072003 \\
\hline $\mathrm{C}$ & 3.33680346 & 5.70379613 & -0.27626173 \\
\hline $\mathrm{C}$ & 1.10939672 & 4.99043643 & 1.60832899 \\
\hline $\mathrm{H}$ & 0.05338080 & 4.77009090 & 1.77910603 \\
\hline $\mathrm{H}$ & 1.58120915 & 4.99914094 & 2.59743590 \\
\hline $\mathrm{C}$ & 2.67444179 & 6.75549589 & 0.66948926 \\
\hline $\mathrm{C}$ & 1.21906864 & 6.38965382 & 0.99180252 \\
\hline $\mathrm{H}$ & 0.78359950 & 7.12146296 & 1.68106099 \\
\hline $\mathrm{H}$ & 0.61784424 & 6.44169194 & 0.07464790 \\
\hline $\mathrm{C}$ & 2.66239852 & 5.71541318 & -1.67456484 \\
\hline $\mathrm{H}$ & 2.41357652 & 6.72769394 & -2.00491364 \\
\hline $\mathrm{H}$ & 3.32062377 & 5.28699855 & -2.43521457 \\
\hline $\mathrm{H}$ & 1.73000714 & 5.15306549 & -1.70371897 \\
\hline $\mathrm{H}$ & 3.23062386 & 6.70998915 & 1.61569449 \\
\hline $\mathrm{C}$ & 2.77881234 & 8.23642545 & 0.18922744 \\
\hline $\mathrm{H}$ & 2.07265643 & 8.39112006 & -0.63247370 \\
\hline $\mathrm{C}$ & 4.81274973 & 6.14138059 & -0.45839143 \\
\hline $\mathrm{H}$ & 5.32667145 & 6.14857435 & 0.50769027 \\
\hline $\mathrm{H}$ & 5.35423889 & 5.43018086 & -1.08769332 \\
\hline $\mathrm{C}$ & 4.93782815 & 7.53090942 & -1.09441006 \\
\hline $\mathrm{H}$ & 4.52817962 & 7.49838737 & -2.11116698 \\
\hline $\mathrm{C}$ & 4.18748600 & 8.61183543 & -0.32023926 \\
\hline $\mathrm{H}$ & 5.99300662 & 7.80983393 & -1.20538838 \\
\hline $\mathrm{C}$ & 4.89789742 & 9.15945559 & 0.94266646 \\
\hline $\mathrm{H}$ & 5.18537325 & 8.33947481 & 1.60493270 \\
\hline $\mathrm{C}$ & 2.47243843 & 9.22698574 & 1.36385725 \\
\hline $\mathrm{H}$ & 2.28515288 & 8.61867798 & 2.25429596 \\
\hline $\mathrm{C}$ & 3.78072093 & 10.02166638 & 1.64652656 \\
\hline $\mathrm{H}$ & 3.74604514 & 11.01512792 & 1.19353972 \\
\hline $\mathrm{H}$ & 3.98566430 & 10.14426971 & 2.71060079 \\
\hline $\mathrm{O}$ & 4.09484584 & 9.82121815 & -1.18365719 \\
\hline $\mathrm{H}$ & 3.84971339 & 9.55944576 & -2.08158114 \\
\hline $\mathrm{C}$ & 6.06257915 & 10.02082215 & 0.69116970 \\
\hline $\mathrm{O}$ & 6.18175268 & 10.69843503 & -0.35738050 \\
\hline $\mathrm{H}$ & 6.85447778 & 10.15390011 & 1.43388248 \\
\hline $\mathrm{C}$ & 1.21987188 & 10.12533815 & 1.19328113 \\
\hline $\mathrm{H}$ & 0.39107980 & 9.42746721 & 1.01340500 \\
\hline $\mathrm{C}$ & 1.26453263 & 11.09292261 & 0.00264481 \\
\hline $\mathrm{H}$ & 2.03163608 & 11.86575103 & 0.12419946 \\
\hline $\mathrm{H}$ & 1.44996769 & 10.58432381 & -0.94705875 \\
\hline $\mathrm{H}$ & 0.30549062 & 11.61103777 & -0.08573062 \\
\hline $\mathrm{C}$ & 0.90883196 & 10.88064917 & 2.49422983 \\
\hline $\mathrm{H}$ & -0.03837571 & 11.42029407 & 2.40905457 \\
\hline $\mathrm{H}$ & 0.82808083 & 10.19919298 & 3.34743031 \\
\hline $\mathrm{H}$ & 1.68201152 & 11.62202832 & 2.72743359 \\
\hline $\mathrm{C}$ & -1.97335684 & -3.12234798 & -0.33616726 \\
\hline $\mathrm{H}$ & -2.94688468 & -3.32378104 & -0.78085785 \\
\hline $\mathrm{H}$ & -1.20928398 & -3.73736588 & -0.82055842 \\
\hline $\mathrm{H}$ & -1.98138493 & -3.38999432 & 0.72401 \\
\hline & & & \\
\hline
\end{tabular}

XI (Fig. 8S), E = -1552.4116

$\begin{array}{lrrr}11 & & & \\ \mathrm{C} & 0.00000000 & 0.00000000 & 0.00000000 \\ \mathrm{C} & 1.54678531 & 0.00000000 & 0.00000000 \\ \mathrm{C} & 1.98527864 & 1.50705346 & 0.00000000 \\ \mathrm{C} & 1.39210963 & 2.43035150 & 1.12048443 \\ \mathrm{C} & -0.15330914 & 2.27528312 & 1.08052393 \\ \mathrm{C} & -0.62576949 & 0.81764067 & 1.12270238\end{array}$




\begin{tabular}{|c|c|c|c|}
\hline $\mathrm{H}$ & -0.35148407 & 0.39821062 & -0.95775045 \\
\hline $\mathrm{H}$ & -0.61362382 & 2.81260139 & 1.91588165 \\
\hline $\mathrm{H}$ & -0.53852187 & 2.73901093 & 0.16210966 \\
\hline $\mathrm{H}$ & -0.39719445 & 0.35168175 & 2.08715325 \\
\hline $\mathrm{H}$ & -1.71323371 & 0.78647212 & 1.00983391 \\
\hline $\mathrm{H}$ & 1.54245810 & 1.90055927 & -0.92990494 \\
\hline $\mathrm{C}$ & 1.86411911 & 2.08360925 & 2.55539729 \\
\hline $\mathrm{H}$ & 2.87304652 & 1.67085562 & 2.59723036 \\
\hline $\mathrm{H}$ & 1.84037023 & 2.96284294 & 3.20413692 \\
\hline $\mathrm{H}$ & 1.20819191 & 1.34468898 & 3.01816553 \\
\hline $\mathrm{C}$ & 1.73764109 & 3.91768023 & 0.70232276 \\
\hline $\mathrm{H}$ & 1.22246048 & 4.01475728 & -0.26081146 \\
\hline $\mathrm{C}$ & 3.49754112 & 1.72876746 & -0.14493644 \\
\hline $\mathrm{H}$ & 3.90714629 & 1.06277452 & -0.90872401 \\
\hline $\mathrm{H}$ & 4.02422890 & 1.47657282 & 0.78139613 \\
\hline $\mathrm{C}$ & 3.80022271 & 3.17196526 & -0.57071845 \\
\hline $\mathrm{H}$ & 3.36541184 & 3.31145193 & -1.56527276 \\
\hline $\mathrm{H}$ & 4.88367280 & 3.29414305 & -0.68613252 \\
\hline $\mathrm{C}$ & 3.24825292 & 4.24654017 & 0.40104284 \\
\hline $\mathrm{C}$ & 2.00371638 & -0.66340045 & -1.31947784 \\
\hline $\mathrm{H}$ & 3.07999524 & -0.85391539 & -1.33254594 \\
\hline $\mathrm{H}$ & 1.50414048 & -1.62796709 & -1.43921523 \\
\hline $\mathrm{H}$ & 1.75562422 & -0.04522115 & -2.18965253 \\
\hline $\mathrm{C}$ & 2.11978332 & -0.83491329 & 1.16281218 \\
\hline $\mathrm{H}$ & 1.72894160 & -0.54600399 & 2.13873409 \\
\hline $\mathrm{H}$ & 1.87656356 & -1.88960908 & 1.01511892 \\
\hline $\mathrm{H}$ & 3.21001443 & -0.74885386 & 1.19917783 \\
\hline $\mathrm{O}$ & -0.45280367 & -1.37248616 & 0.08652561 \\
\hline $\mathrm{C}$ & -1.63978367 & -1.66257430 & -0.49861908 \\
\hline $\mathrm{O}$ & -2.32268953 & -0.84909381 & -1.07076010 \\
\hline $\mathrm{C}$ & 4.11896176 & 4.19992799 & 1.68128018 \\
\hline $\mathrm{H}$ & 5.17271302 & 4.37582830 & 1.45026699 \\
\hline $\mathrm{H}$ & 3.81907260 & 4.92085458 & 2.44515945 \\
\hline $\mathrm{H}$ & 4.07552038 & 3.22029110 & 2.15026353 \\
\hline $\mathrm{C}$ & 3.33788109 & 5.70505101 & -0.27610334 \\
\hline $\mathrm{C}$ & 1.10784579 & 4.98998594 & 1.60925960 \\
\hline $\mathrm{H}$ & 0.05124633 & 4.76991302 & 1.77764516 \\
\hline $\mathrm{H}$ & 1.57735700 & 4.99654646 & 2.59974389 \\
\hline $\mathrm{C}$ & 2.67571425 & 6.75686335 & 0.67148015 \\
\hline $\mathrm{C}$ & 1.22117150 & 6.39015831 & 0.99539087 \\
\hline $\mathrm{H}$ & 0.78765352 & 7.12179599 & 1.68677093 \\
\hline $\mathrm{H}$ & 0.61842866 & 6.44620502 & 0.07943596 \\
\hline $\mathrm{C}$ & 2.65880401 & 5.71838210 & -1.67186295 \\
\hline $\mathrm{H}$ & 2.43070337 & 6.73289239 & -2.00789363 \\
\hline $\mathrm{H}$ & 3.30768527 & 5.27428647 & -2.43131796 \\
\hline $\mathrm{H}$ & 1.71600280 & 5.17285784 & -1.69501960 \\
\hline $\mathrm{H}$ & 3.23101551 & 6.70739788 & 1.61934580 \\
\hline $\mathrm{C}$ & 2.78614032 & 8.23067405 & 0.18502044 \\
\hline $\mathrm{H}$ & 2.08461034 & 8.38348899 & -0.64059503 \\
\hline $\mathrm{C}$ & 4.81404569 & 6.14105876 & -0.46691900 \\
\hline $\mathrm{H}$ & 5.33250173 & 6.13777732 & 0.49761201 \\
\hline $\mathrm{H}$ & 5.34672532 & 5.42538369 & -1.09941219 \\
\hline $\mathrm{C}$ & 4.94625791 & 7.53310246 & -1.09533136 \\
\hline $\mathrm{H}$ & 4.54368411 & 7.51742423 & -2.11440311 \\
\hline $\mathrm{C}$ & 4.19942665 & 8.63029036 & -0.33166126 \\
\hline $\mathrm{H}$ & 6.00110719 & 7.81198705 & -1.20468315 \\
\hline $\mathrm{C}$ & 4.88081185 & 9.08864904 & 0.97625024 \\
\hline $\mathrm{H}$ & 5.01063294 & 8.26068047 & 1.68064199 \\
\hline $\mathrm{C}$ & 2.47198689 & 9.23477402 & 1.34405744 \\
\hline $\mathrm{H}$ & 2.29114649 & 8.64315056 & 2.24784181 \\
\hline $\mathrm{C}$ & 3.77214938 & 10.04801811 & 1.62596303 \\
\hline $\mathrm{H}$ & 3.78068494 & 10.99996072 & 1.09547697 \\
\hline $\mathrm{H}$ & 3.95171796 & 10.21804938 & 2.68812563 \\
\hline
\end{tabular}




$\begin{array}{lrcc}\mathrm{O} & 4.12923537 & 9.82529692 & -1.11132586 \\ \mathrm{H} & 3.72808402 & 9.61096967 & -1.96143210 \\ \mathrm{C} & 6.09566013 & 9.85558927 & 0.97603880 \\ \mathrm{O} & 6.52959204 & 10.49299036 & -0.04260316 \\ \mathrm{H} & 6.66147930 & 9.95596262 & 1.90729694 \\ \mathrm{C} & 1.22200354 & 10.13627910 & 1.16195600 \\ \mathrm{H} & 0.39739405 & 9.43174105 & 0.98696921 \\ \mathrm{C} & 1.26975291 & 11.08999924 & -0.03877185 \\ \mathrm{H} & 2.03357876 & 11.86607954 & 0.07903452 \\ \mathrm{H} & 1.46834014 & 10.57119731 & -0.97921464 \\ \mathrm{H} & 0.30781426 & 11.60096872 & -0.13861773 \\ \mathrm{C} & 0.90344922 & 10.90352164 & 2.45404487 \\ \mathrm{H} & -0.04831873 & 11.43396963 & 2.36142363 \\ \mathrm{H} & 0.82727617 & 10.23203294 & 3.31572838 \\ \mathrm{H} & 1.66941036 & 11.65528082 & 2.67839255 \\ \mathrm{C} & -1.97139796 & -3.12438135 & -0.33500617 \\ \mathrm{H} & -2.94376309 & -3.32739637 & -0.78154668 \\ \mathrm{H} & -1.20542923 & -3.73872918 & -0.81722575 \\ \mathrm{H} & -1.98144832 & -3.39134896 & 0.72535525 \\ \mathrm{H} & 7.32714068 & 11.02048507 & 0.14528565\end{array}$

XII (Fig. 8S), input structure

$\begin{array}{lrrr}11 & & & \\ \mathrm{C} & 0.00000000 & 0.00000000 & 0.00000000 \\ \mathrm{C} & 1.54673259 & 0.00000000 & 0.00000000 \\ \mathrm{C} & 1.98550759 & 1.50708823 & 0.00000000 \\ \mathrm{C} & 1.39141921 & 2.43060888 & 1.11977499 \\ \mathrm{C} & -0.15413609 & 2.27565861 & 1.07923394 \\ \mathrm{C} & -0.62568147 & 0.81783406 & 1.12255158 \\ \mathrm{H} & -0.35106994 & 0.39846893 & -0.95781373 \\ \mathrm{H} & -0.61497020 & 2.81353204 & 1.91397515 \\ \mathrm{H} & -0.53946814 & 2.73813099 & 0.16017605 \\ \mathrm{H} & -0.39607212 & 0.35282910 & 2.08705921 \\ \mathrm{H} & -1.71315011 & 0.78617770 & 1.01044174 \\ \mathrm{H} & 1.54306141 & 1.90028094 & -0.93032180 \\ \mathrm{C} & 1.86276380 & 2.08468734 & 2.55504443 \\ \mathrm{H} & 2.87534483 & 1.68120411 & 2.59806732 \\ \mathrm{H} & 1.82855415 & 2.96195778 & 3.20598269 \\ \mathrm{H} & 1.21267467 & 1.33840314 & 3.01387201 \\ \mathrm{C} & 1.73754571 & 3.91775049 & 0.70138885 \\ \mathrm{H} & 1.22184221 & 4.01449601 & -0.26149942 \\ \mathrm{C} & 3.49810830 & 1.72844728 & -0.14353892 \\ \mathrm{H} & 3.90859363 & 1.06130829 & -0.90565338 \\ \mathrm{H} & 4.02326848 & 1.47835018 & 0.78400912 \\ \mathrm{C} & 3.80086117 & 3.17126987 & -0.57132750 \\ \mathrm{H} & 3.36474863 & 3.30898277 & -1.56567855 \\ \mathrm{H} & 4.88437953 & 3.29366949 & -0.68744968 \\ \mathrm{C} & 3.24867992 & 4.24523198 & 0.40092363 \\ \mathrm{C} & 2.00301153 & -0.66275428 & -1.32010037 \\ \mathrm{H} & 3.07960359 & -0.85137283 & -1.33534368 \\ \mathrm{H} & 1.50502874 & -1.62826468 & -1.43861879 \\ \mathrm{H} & 1.75164815 & -0.04531600 & -2.18992010 \\ \mathrm{C} & 2.11993220 & -0.83604200 & 1.16228372 \\ \mathrm{H} & 1.73091355 & -0.54783077 & 2.13922982 \\ \mathrm{H} & 1.87488998 & -1.89024966 & 1.01421715 \\ \mathrm{H} & 3.21035021 & -0.75251506 & 1.19801766 \\ \mathrm{O} & -0.45283086 & -1.37187152 & 0.08638243 \\ \mathrm{C} & -1.64009187 & -1.66113300 & -0.49973341 \\ \mathrm{O} & -2.32111613 & -0.84652953 & -1.07240303 \\ \mathrm{C} & 4.11912652 & 4.20033514 & 1.68090660 \\ \mathrm{H} & 5.17348300 & 4.37299548 & 1.44989858 \\ \mathrm{H} & 3.82096367 & 4.92284502 & 2.44391456\end{array}$




\begin{tabular}{lrrr}
$\mathrm{H}$ & 4.07305564 & 3.22150386 & 2.15072003 \\
$\mathrm{C}$ & 3.33680346 & 5.70379613 & -0.27626173 \\
$\mathrm{C}$ & 1.10939672 & 4.99043643 & 1.60832899 \\
$\mathrm{H}$ & 0.05338080 & 4.77009090 & 1.77910603 \\
$\mathrm{H}$ & 1.58120915 & 4.99914094 & 2.59743590 \\
$\mathrm{C}$ & 2.67444179 & 6.75549589 & 0.66948926 \\
$\mathrm{C}$ & 1.21906864 & 6.38965382 & 0.99180252 \\
$\mathrm{H}$ & 0.78359950 & 7.12146296 & 1.68106099 \\
$\mathrm{H}$ & 0.61784424 & 6.44169194 & 0.07464790 \\
$\mathrm{C}$ & 2.66239852 & 5.71541318 & -1.67456484 \\
$\mathrm{H}$ & 2.41357652 & 6.72769394 & -2.00491364 \\
$\mathrm{H}$ & 3.32062377 & 5.28699855 & -2.43521457 \\
$\mathrm{H}$ & 1.73000714 & 5.15306549 & -1.70371897 \\
$\mathrm{H}$ & 3.23062386 & 6.70998915 & 1.61569449 \\
$\mathrm{C}$ & 2.77881234 & 8.23642545 & 0.18922744 \\
$\mathrm{H}$ & 2.07265643 & 8.39112006 & -0.63247370 \\
$\mathrm{C}$ & 4.81274973 & 6.14138059 & -0.45839143 \\
$\mathrm{H}$ & 5.32667145 & 6.14857435 & 0.50769027 \\
$\mathrm{H}$ & 5.35423889 & 5.43018086 & -1.08769332 \\
$\mathrm{C}$ & 4.93782815 & 7.53090942 & -1.09441006 \\
$\mathrm{H}$ & 4.52817962 & 7.49838737 & -2.11116698 \\
$\mathrm{C}$ & 4.18748600 & 8.61183543 & -0.32023926 \\
$\mathrm{H}$ & 5.99300662 & 7.80983393 & -1.20538838 \\
$\mathrm{C}$ & 4.89789742 & 9.15945559 & 0.94266646 \\
$\mathrm{H}$ & 5.18537325 & 8.33947481 & 1.60493270 \\
$\mathrm{C}$ & 2.47243843 & 9.22698574 & 1.36385725 \\
$\mathrm{H}$ & 2.28515288 & 8.61867798 & 2.25429596 \\
$\mathrm{C}$ & 3.78072093 & 10.02166638 & 1.64652656 \\
$\mathrm{H}$ & 3.74604514 & 11.01512792 & 1.19353972 \\
$\mathrm{H}$ & 3.98566430 & 10.14426971 & 2.71060079 \\
$\mathrm{O}$ & 4.09484584 & 9.82121815 & -1.18365719 \\
$\mathrm{C}$ & 6.06257915 & 10.02082215 & 0.69116970 \\
$\mathrm{O}$ & 6.18175268 & 10.69843503 & -0.35738050 \\
$\mathrm{H}$ & 6.85447778 & 10.15390011 & 1.43388248 \\
$\mathrm{C}$ & 1.21987188 & 10.12533815 & 1.19328113 \\
$\mathrm{H}$ & 0.39107980 & 9.42746721 & 1.01340500 \\
$\mathrm{C}$ & 1.26453263 & 11.09292261 & 0.00264481 \\
$\mathrm{H}$ & 2.03163608 & 11.86575103 & 0.12419946 \\
$\mathrm{H}$ & 1.44996769 & 10.58432381 & -0.94705875 \\
$\mathrm{H}$ & 0.30549062 & 11.61103777 & -0.08573062 \\
$\mathrm{C}$ & 0.90883196 & 10.88064917 & 2.49422983 \\
$\mathrm{H}$ & -0.03837571 & 11.42029407 & 2.40905457 \\
$\mathrm{H}$ & 0.82808083 & 10.19919298 & 3.34743031 \\
$\mathrm{H}$ & 1.68201152 & 11.62202832 & 2.72743359 \\
$\mathrm{C}$ & -1.97335684 & -3.12234798 & -0.33616726 \\
$\mathrm{H}$ & -2.94688468 & -3.32378104 & -0.78085785 \\
$\mathrm{H}$ & -1.20928398 & -3.73736588 & -0.82055842 \\
$\mathrm{H}$ & -1.98138493 & -3.38999432 & 0.72401445 \\
& 6.51166302 & 11.57276550 & -0.13759561 \\
& 4.27027675 & 10.68959045 & -0.81385515 \\
\hline
\end{tabular}

XII (Fig. 8S), output structure, E = -1552.4262

$\begin{array}{lrrr}11 & & & \\ \mathrm{C} & 0.00000000 & 0.00000000 & 0.00000000 \\ \mathrm{C} & 1.54711864 & 0.00000000 & 0.00000000 \\ \mathrm{C} & 1.98535583 & 1.50649876 & 0.00000000 \\ \mathrm{C} & 1.39315907 & 2.42881824 & 1.12177631 \\ \mathrm{C} & -0.15248338 & 2.27649274 & 1.07919263 \\ \mathrm{C} & -0.62713223 & 0.81964614 & 1.12090823 \\ \mathrm{H} & -0.35147287 & 0.39767540 & -0.95820569 \\ \mathrm{H} & -0.61373532 & 2.81455656 & 1.91370341 \\ \mathrm{H} & -0.53442883 & 2.74083084 & 0.15950369 \\ \mathrm{H} & -0.40278001 & 0.35434219 & 2.08649226\end{array}$




\begin{tabular}{|c|c|c|c|}
\hline $\mathrm{H}$ & -1.71415421 & 0.79076169 & 1.00471328 \\
\hline $\mathrm{H}$ & 1.54151548 & 1.90019478 & -0.92953682 \\
\hline $\mathrm{C}$ & 1.86463710 & 2.08199932 & 2.55654680 \\
\hline $\mathrm{H}$ & 2.87158606 & 1.66451586 & 2.59829417 \\
\hline $\mathrm{H}$ & 1.84483054 & 2.96140592 & 3.20533795 \\
\hline $\mathrm{H}$ & 1.20597921 & 1.34624056 & 3.02023561 \\
\hline $\mathrm{C}$ & 1.73948171 & 3.91559934 & 0.70413887 \\
\hline $\mathrm{H}$ & 1.22590524 & 4.01156529 & -0.26029074 \\
\hline $\mathrm{C}$ & 3.49746182 & 1.72780629 & -0.14790944 \\
\hline $\mathrm{H}$ & 3.90497775 & 1.06395341 & -0.91445060 \\
\hline $\mathrm{H}$ & 4.02766080 & 1.47390661 & 0.77577083 \\
\hline $\mathrm{C}$ & 3.79973605 & 3.17151272 & -0.57251530 \\
\hline $\mathrm{H}$ & 3.36131728 & 3.31281835 & -1.56547464 \\
\hline $\mathrm{H}$ & 4.88307579 & 3.29228850 & -0.69065561 \\
\hline $\mathrm{C}$ & 3.25063572 & 4.24243087 & 0.40505084 \\
\hline $\mathrm{C}$ & 2.00439176 & -0.66333466 & -1.31930271 \\
\hline $\mathrm{H}$ & 3.08007242 & -0.85705994 & -1.33088864 \\
\hline $\mathrm{H}$ & 1.50268308 & -1.62660710 & -1.43962644 \\
\hline $\mathrm{H}$ & 1.75885531 & -0.04469630 & -2.18990067 \\
\hline $\mathrm{C}$ & 2.12018930 & -0.83470843 & 1.16286040 \\
\hline $\mathrm{H}$ & 1.72745615 & -0.54770517 & 2.13850268 \\
\hline $\mathrm{H}$ & 1.87811490 & -1.88931453 & 1.01346529 \\
\hline $\mathrm{H}$ & 3.21039204 & -0.74815038 & 1.20132910 \\
\hline $\mathrm{O}$ & -0.45238577 & -1.37166282 & 0.08756583 \\
\hline $\mathrm{C}$ & -1.64433146 & -1.65922350 & -0.49060868 \\
\hline $\mathrm{O}$ & -2.32888278 & -0.84230367 & -1.05564045 \\
\hline $\mathrm{C}$ & 4.12721677 & 4.19436836 & 1.68103928 \\
\hline $\mathrm{H}$ & 5.17914876 & 4.37379676 & 1.44498859 \\
\hline $\mathrm{H}$ & 3.83075605 & 4.91069718 & 2.45038372 \\
\hline $\mathrm{H}$ & 4.08708739 & 3.21266343 & 2.14575342 \\
\hline $\mathrm{C}$ & 3.34167796 & 5.70237590 & -0.26760058 \\
\hline $\mathrm{C}$ & 1.10600493 & 4.98917485 & 1.60705059 \\
\hline $\mathrm{H}$ & 0.04762561 & 4.77235304 & 1.76606172 \\
\hline $\mathrm{H}$ & 1.56695500 & 4.99623151 & 2.60130910 \\
\hline $\mathrm{C}$ & 2.68790931 & 6.74586987 & 0.69322873 \\
\hline $\mathrm{C}$ & 1.22553354 & 6.38916530 & 0.99332468 \\
\hline $\mathrm{H}$ & 0.78034985 & 7.12062651 & 1.67708551 \\
\hline $\mathrm{H}$ & 0.63669733 & 6.44276282 & 0.06799832 \\
\hline $\mathrm{C}$ & 2.65694028 & 5.72692148 & -1.66146115 \\
\hline $\mathrm{H}$ & 2.42695331 & 6.74304992 & -1.99679795 \\
\hline $\mathrm{H}$ & 3.30119457 & 5.28350899 & -2.42509893 \\
\hline $\mathrm{H}$ & 1.71339709 & 5.18393523 & -1.68332779 \\
\hline $\mathrm{H}$ & 3.23689996 & 6.68742772 & 1.63877957 \\
\hline $\mathrm{C}$ & 2.80237801 & 8.23512941 & 0.23802839 \\
\hline $\mathrm{H}$ & 2.01609016 & 8.40947062 & -0.51716780 \\
\hline $\mathrm{C}$ & 4.81655371 & 6.13683937 & -0.45826427 \\
\hline $\mathrm{H}$ & 5.32973309 & 6.18342238 & 0.50436009 \\
\hline $\mathrm{H}$ & 5.35949455 & 5.41669235 & -1.07480894 \\
\hline $\mathrm{C}$ & 4.92831461 & 7.50927888 & -1.12373722 \\
\hline $\mathrm{H}$ & 4.61053198 & 7.45642175 & -2.17731958 \\
\hline $\mathrm{C}$ & 4.04505274 & 8.54406659 & -0.52834758 \\
\hline $\mathrm{H}$ & 5.95161504 & 7.89391405 & -1.16201659 \\
\hline $\mathrm{C}$ & 5.00027697 & 9.02856732 & 1.94761239 \\
\hline $\mathrm{H}$ & 4.94759505 & 8.08462750 & 2.47975527 \\
\hline $\mathrm{C}$ & 2.51791145 & 9.24053655 & 1.42800957 \\
\hline $\mathrm{H}$ & 2.17200472 & 8.58947422 & 2.23608776 \\
\hline $\mathrm{C}$ & 3.80226649 & 9.93041918 & 1.96164692 \\
\hline $\mathrm{H}$ & 4.03864258 & 10.85020476 & 1.41882283 \\
\hline $\mathrm{H}$ & 3.59930010 & 10.24740850 & 2.99053541 \\
\hline $\mathrm{O}$ & 4.25875832 & 9.73482368 & -0.98923443 \\
\hline $\mathrm{C}$ & 6.20290609 & 9.36332349 & 1.44206712 \\
\hline $\mathrm{O}$ & 6.40545899 & 10.53590941 & 0.81071686 \\
\hline $\mathrm{H}$ & 7.05545582 & 8.69187450 & 1.51329179 \\
\hline
\end{tabular}




$\begin{array}{lrrr}\mathrm{C} & 1.34498157 & 10.22221958 & 1.15587920 \\ \mathrm{H} & 0.48483690 & 9.59498013 & 0.88681137 \\ \mathrm{C} & 1.57882300 & 11.18859057 & -0.01661009 \\ \mathrm{H} & 2.43958803 & 11.85079486 & 0.15218814 \\ \mathrm{H} & 1.69298984 & 10.67270573 & -0.98006156 \\ \mathrm{H} & 0.71944507 & 11.85244242 & -0.14477885 \\ \mathrm{C} & 0.96166291 & 11.00054276 & 2.42394270 \\ \mathrm{H} & 0.03446801 & 11.55725854 & 2.26242692 \\ \mathrm{H} & 0.80102609 & 10.32828934 & 3.27277599 \\ \mathrm{H} & 1.73123142 & 11.72547636 & 2.70789521 \\ \mathrm{C} & -1.97709144 & -3.12049514 & -0.32815969 \\ \mathrm{H} & -2.94989833 & -3.32217611 & -0.77427596 \\ \mathrm{H} & -1.21181951 & -3.73545344 & -0.81060241 \\ \mathrm{H} & -1.98732824 & -3.38787899 & 0.73212856 \\ \mathrm{H} & 7.32206969 & 10.61428772 & 0.52269771 \\ \mathrm{H} & 3.60262303 & 10.38600438 & -0.67552134\end{array}$

\section{XIII (Fig.9S) E = -1552.4231}

11

C

C

C

C

C

C

$\mathrm{H}$

$\mathrm{H}$

$\mathrm{H}$

$\mathrm{H}$

$\mathrm{H}$

$\mathrm{H}$

C

$\mathrm{H}$

$\mathrm{H}$

$\mathrm{H}$

C

$\mathrm{H}$

C

$\mathrm{H}$

$\mathrm{H}$

C

$\mathrm{H}$

$\mathrm{H}$

C

$\mathrm{C}$

$\mathrm{H}$

$\mathrm{H}$

$\mathrm{H}$

C

$\mathrm{H}$

$\mathrm{H}$

$\mathrm{H}$

$\mathrm{O}$

$\mathrm{C}$

$\mathrm{O}$

$\mathrm{C}$

$\mathrm{H}$

$\mathrm{H}$

$\mathrm{H}$

C

$\mathrm{C}$

$\mathrm{H}$

$\mathrm{H}$
$\begin{array}{llll}-5.16159100 & 0.44168100 & -0.22684200\end{array}$
$\begin{array}{lll}-4.63840600 & -0.98008600 & 0.08695000\end{array}$
$\begin{array}{lll}-3.07532700 & -0.90665100 & -0.02903200\end{array}$
$\begin{array}{lll}-2.34169100 & 0.19350200 & 0.81421700\end{array}$
$\begin{array}{llll}-3.01114900 & 1.55601000 & 0.48332300\end{array}$
$\begin{array}{lll}-4.53732900 & 1.54030400 & 0.62449300\end{array}$
$\begin{array}{llll}-4.96344800 & 0.66447000 & -1.28078300\end{array}$
$\begin{array}{llll}-2.61204900 & 2.34533700 & 1.12850600\end{array}$
$\begin{array}{llll}-2.76041600 & 1.83977400 & -0.54809200\end{array}$
$\begin{array}{lll}-4.84029800 & 1.41243900 & 1.66910600\end{array}$
$\begin{array}{llll}-4.93992200 & 2.50310900 & 0.29747100\end{array}$
$\begin{array}{llll}-2.91259100 & -0.59336400 & -1.07368500\end{array}$
$\begin{array}{lll}-2.42023000 & -0.01242800 & 2.34818100\end{array}$
$\begin{array}{lll}-2.46778900 & -1.06006900 & 2.64850300\end{array}$
$\begin{array}{lll}-1.56253200 & 0.43723200 & 2.85491900\end{array}$
$\begin{array}{lll}-3.30665100 & 0.46831900 & 2.76440500\end{array}$
$\begin{array}{llll}-0.85395700 & 0.24739900 & 0.27665000\end{array}$
$\begin{array}{llll}-0.99706700 & 0.52130900 & -0.77572500\end{array}$
$\begin{array}{lll}-2.36469400 & -2.26108000 & 0.10382900\end{array}$
$\begin{array}{lll}-2.89606100 & -3.02618000 & -0.46765200\end{array}$
$\begin{array}{lll}-2.36866600 & -2.61058200 & 1.14138600\end{array}$
$\begin{array}{lll}-0.93161200 & -2.18369900 & -0.44087900\end{array}$
$\begin{array}{llll}-1.00621700 & -1.97303200 & -1.51241700\end{array}$
$\begin{array}{lll}-0.45894900 & -3.16894500 & -0.34938000\end{array}$
$\begin{array}{lll}-0.05365800 & -1.10832100 & 0.24947800\end{array}$
$\begin{array}{lll}-5.18676900 & -1.92186200 & -1.00938900\end{array}$
$\begin{array}{lll}-5.00524000 & -2.97419200 & -0.77599500\end{array}$
$\begin{array}{lll}-6.26842800 & -1.79370400 & -1.09679100\end{array}$
$\begin{array}{llll}-4.74209500 & -1.70666500 & -1.98762200\end{array}$
$\begin{array}{lll}-5.15772500 & -1.49486400 & 1.44445100\end{array}$
$\begin{array}{lll}-4.96234300 & -0.81215400 & 2.27170300\end{array}$
$\begin{array}{lll}-6.23894700 & -1.64141100 & 1.39366300\end{array}$
$\begin{array}{lll}-4.70472500 & -2.45988100 & 1.69130300\end{array}$
$\begin{array}{llll}-6.59743700 & 0.44488500 & -0.04950100\end{array}$
$\begin{array}{llll}-7.30498700 & 1.31113700 & -0.81579300\end{array}$
$\begin{array}{lll}-6.80263100 & 2.06233800 & -1.61483400\end{array}$
$\begin{array}{lll}0.27560800 & -1.62374200 & 1.67246000\end{array}$
$\begin{array}{lll}0.79166500 & -2.58672200 & 1.63757000\end{array}$
$\begin{array}{lll}0.88773000 & -0.93756500 & 2.26181900\end{array}$
$\begin{array}{lll}-0.63211800 & -1.79273500 & 2.24575100\end{array}$
$\begin{array}{lll}1.30651400 & -0.88831400 & -0.58313600\end{array}$
$\begin{array}{lll}-0.00516200 & 1.38118900 & 0.87858900\end{array}$
$\begin{array}{lll}-0.55740600 & 2.32273700 & 0.84865200\end{array}$
$\begin{array}{llll}0.21749400 & 1.19365100 & 1.93511400\end{array}$ 


\begin{tabular}{|c|c|c|c|}
\hline $\mathrm{C}$ & 2.12571300 & 0.26935800 & 0.07054000 \\
\hline $\mathrm{C}$ & 1.31308100 & 1.57058100 & 0.11864100 \\
\hline $\mathrm{H}$ & 1.89132300 & 2.36907200 & 0.59582600 \\
\hline $\mathrm{H}$ & 1.10928300 & 1.91393000 & -0.90416000 \\
\hline $\mathrm{C}$ & 1.01394000 & -0.58436500 & -2.07766400 \\
\hline $\mathrm{H}$ & 1.86707000 & -0.12292800 & -2.58484300 \\
\hline $\mathrm{H}$ & 0.77495200 & -1.49811600 & -2.62831500 \\
\hline $\mathrm{H}$ & 0.17900700 & 0.10045300 & -2.21734100 \\
\hline $\mathrm{H}$ & 2.31698300 & -0.03257800 & 1.10611600 \\
\hline $\mathrm{C}$ & 3.53653200 & 0.52981300 & -0.54802100 \\
\hline $\mathrm{H}$ & 3.40819300 & 1.12812300 & -1.46585200 \\
\hline $\mathrm{C}$ & 2.19971700 & -2.15282900 & -0.52176400 \\
\hline $\mathrm{H}$ & 2.47528100 & -2.37861900 & 0.51042300 \\
\hline $\mathrm{H}$ & 1.67173300 & -3.02655300 & -0.91058100 \\
\hline $\mathrm{C}$ & 3.48646400 & -1.97811600 & -1.33008900 \\
\hline $\mathrm{H}$ & 3.25616100 & -1.94342500 & -2.40766100 \\
\hline $\mathrm{C}$ & 4.19828800 & -0.69787500 & -1.06658600 \\
\hline $\mathrm{H}$ & 4.17987600 & -2.81913900 & -1.20863600 \\
\hline $\mathrm{C}$ & 5.20807800 & -0.80850000 & 1.37830500 \\
\hline $\mathrm{H}$ & 4.38830700 & -0.92843300 & 2.08016800 \\
\hline $\mathrm{C}$ & 4.44706700 & 1.39713100 & 0.41820000 \\
\hline $\mathrm{H}$ & 3.78290300 & 1.62696900 & 1.25809800 \\
\hline $\mathrm{C}$ & 5.62235100 & 0.58845500 & 1.02707300 \\
\hline $\mathrm{H}$ & 6.47781800 & 0.59100900 & 0.34468100 \\
\hline $\mathrm{H}$ & 5.95495200 & 1.10985100 & 1.93080600 \\
\hline $\mathrm{O}$ & 5.36318700 & -0.52076100 & -1.61998300 \\
\hline $\mathrm{C}$ & 5.80081800 & -1.94841800 & 0.96859900 \\
\hline $\mathrm{O}$ & 6.85955700 & -2.06017700 & 0.13700800 \\
\hline $\mathrm{H}$ & 5.42685300 & -2.92630000 & 1.25545500 \\
\hline $\mathrm{C}$ & 4.86456400 & 2.76914500 & -0.17442400 \\
\hline $\mathrm{H}$ & 3.93050900 & 3.23811700 & -0.51251500 \\
\hline $\mathrm{C}$ & 5.80019000 & 2.69096800 & -1.38936900 \\
\hline $\mathrm{H}$ & 6.78057400 & 2.27869800 & -1.12749700 \\
\hline $\mathrm{H}$ & 5.38900200 & 2.08480300 & -2.20231200 \\
\hline $\mathrm{H}$ & 5.97287600 & 3.69432300 & -1.78832300 \\
\hline $\mathrm{C}$ & 5.45867100 & 3.68321000 & 0.90880500 \\
\hline $\mathrm{H}$ & 5.59275800 & 4.69539500 & 0.51747900 \\
\hline $\mathrm{H}$ & 4.80587100 & 3.75088400 & 1.78542200 \\
\hline $\mathrm{H}$ & 6.44146000 & 3.33537800 & 1.24411700 \\
\hline $\mathrm{C}$ & -8.78018300 & 1.19498900 & -0.52840100 \\
\hline $\mathrm{H}$ & -9.32501000 & 1.91864900 & -1.13298300 \\
\hline $\mathrm{H}$ & -9.12818700 & 0.18316500 & -0.75571300 \\
\hline $\mathrm{H}$ & -8.97120300 & 1.37295500 & 0.53350200 \\
\hline $\mathrm{H}$ & 7.33368100 & -1.21838300 & 0.08688300 \\
\hline $\mathrm{H}$ & 5.74387300 & -1.36108400 & -1.93385400 \\
\hline
\end{tabular}

(Fig.11) input structure

$\begin{array}{lrrr}11 & & & \\ \mathrm{C} & -5.12453800 & 0.46620500 & -0.25168100 \\ \mathrm{C} & -4.60888700 & -0.95240200 & 0.08778600 \\ \mathrm{C} & -3.04478500 & -0.88674100 & -0.01643400 \\ \mathrm{C} & -2.31357000 & 0.22389500 & 0.81517200 \\ \mathrm{C} & -2.97440400 & 1.58319400 & 0.45513100 \\ \mathrm{C} & -4.50165900 & 1.57679300 & 0.58507600 \\ \mathrm{H} & -4.91897700 & 0.67150700 & -1.30782300 \\ \mathrm{H} & -2.57671400 & 2.38245400 & 1.08892900 \\ \mathrm{H} & -2.71417600 & 1.84678600 & -0.57933500 \\ \mathrm{H} & -4.81284900 & 1.46891700 & 1.62945000 \\ \mathrm{H} & -4.89740900 & 2.53540800 & 0.23809900 \\ \mathrm{H} & -2.87221100 & -0.59078700 & -1.06460300 \\ \mathrm{C} & -2.40559100 & 0.04389100 & 2.35149000 \\ \mathrm{H} & -2.46069800 & -0.99849500 & 2.66831200 \\ \mathrm{H} & -1.54947300 & 0.49770900 & 2.85724600\end{array}$




\begin{tabular}{|c|c|c|c|}
\hline $\mathrm{H}$ & -3.29269800 & 0.53584100 & 2.75299500 \\
\hline $\mathrm{C}$ & -0.82116100 & 0.26294500 & 0.28975200 \\
\hline $\mathrm{H}$ & -0.95395700 & 0.52169700 & -0.76784300 \\
\hline $\mathrm{C}$ & -2.34045900 & -2.24167200 & 0.14295800 \\
\hline $\mathrm{H}$ & -2.87080100 & -3.01364200 & -0.42020600 \\
\hline $\mathrm{H}$ & -2.35297700 & -2.57489900 & 1.18582100 \\
\hline $\mathrm{C}$ & -0.90365100 & -2.17734700 & -0.39313300 \\
\hline H & -0.97025700 & -1.98059600 & -1.46784200 \\
\hline $\mathrm{H}$ & -0.43525700 & -3.16285600 & -0.28567700 \\
\hline $\mathrm{C}$ & -0.02596800 & -1.09629300 & 0.28878100 \\
\hline $\mathrm{C}$ & -5.15193500 & -1.90850500 & -0.99878500 \\
\hline $\mathrm{H}$ & -4.97638700 & -2.95785600 & -0.74819200 \\
\hline $\mathrm{H}$ & -6.23234600 & -1.77757800 & -1.09704300 \\
\hline $\mathrm{H}$ & -4.69835800 & -1.70979300 & -1.97639700 \\
\hline $\mathrm{C}$ & -5.14093700 & -1.44517300 & 1.44848100 \\
\hline $\mathrm{H}$ & -4.94912600 & -0.75124400 & 2.26711400 \\
\hline $\mathrm{H}$ & -6.22234900 & -1.58794900 & 1.39124300 \\
\hline $\mathrm{H}$ & -4.69402800 & -2.40830600 & 1.71336100 \\
\hline $\mathrm{O}$ & -6.56172100 & 0.47732700 & -0.08386700 \\
\hline $\mathrm{C}$ & -7.26081500 & 1.33598100 & -0.86597300 \\
\hline $\mathrm{O}$ & -6.75046100 & 2.07542900 & -1.67094900 \\
\hline $\mathrm{C}$ & 0.28969100 & -1.59487200 & 1.72078300 \\
\hline $\mathrm{H}$ & 0.79647400 & -2.56296200 & 1.69958400 \\
\hline $\mathrm{H}$ & 0.90659300 & -0.90774400 & 2.30404400 \\
\hline $\mathrm{H}$ & -0.62271100 & -1.74613700 & 2.29170900 \\
\hline $\mathrm{C}$ & 1.34236000 & -0.89547000 & -0.53555900 \\
\hline $\mathrm{C}$ & 0.02687900 & 1.40346200 & 0.88064400 \\
\hline $\mathrm{H}$ & -0.52140000 & 2.34653300 & 0.83094300 \\
\hline $\mathrm{H}$ & 0.24071600 & 1.23241700 & 1.94181900 \\
\hline $\mathrm{C}$ & 2.15844200 & 0.26884900 & 0.11086100 \\
\hline $\mathrm{C}$ & 1.35144200 & 1.57434300 & 0.12748200 \\
\hline $\mathrm{H}$ & 1.92840900 & 2.38106700 & 0.59337900 \\
\hline $\mathrm{H}$ & 1.15578700 & 1.89876600 & -0.90319800 \\
\hline $\mathrm{C}$ & 1.06215500 & -0.60966600 & -2.03626500 \\
\hline $\mathrm{H}$ & 1.92389900 & -0.16758400 & -2.54599300 \\
\hline $\mathrm{H}$ & 0.81837100 & -1.52957500 & -2.57399800 \\
\hline $\mathrm{H}$ & 0.23539400 & 0.08151600 & -2.19220900 \\
\hline $\mathrm{H}$ & 2.33734400 & -0.01546800 & 1.15298800 \\
\hline $\mathrm{C}$ & 3.57388400 & 0.51184100 & -0.50206800 \\
\hline $\mathrm{H}$ & 3.43523800 & 1.09746200 & -1.42769600 \\
\hline $\mathrm{C}$ & 2.23261400 & -2.16084200 & -0.45401100 \\
\hline $\mathrm{H}$ & 2.49935900 & -2.37544800 & 0.58279200 \\
\hline $\mathrm{H}$ & 1.70250000 & -3.03682900 & -0.83499700 \\
\hline $\mathrm{C}$ & 3.52586600 & -2.00437000 & -1.25522900 \\
\hline $\mathrm{H}$ & 3.31344000 & -1.99369600 & -2.33623600 \\
\hline $\mathrm{C}$ & 4.23757700 & -0.72399900 & -1.01121100 \\
\hline $\mathrm{H}$ & 4.22691700 & -2.83291300 & -1.11885400 \\
\hline $\mathrm{C}$ & 5.12604900 & -0.80517000 & 1.58460700 \\
\hline $\mathrm{H}$ & 4.49429900 & -0.88545300 & 2.46291200 \\
\hline $\mathrm{C}$ & 4.48954800 & 1.38320100 & 0.45193000 \\
\hline $\mathrm{H}$ & 3.80495600 & 1.70105700 & 1.24365300 \\
\hline $\mathrm{C}$ & 5.59574100 & 0.55348800 & 1.15775400 \\
\hline $\mathrm{H}$ & 6.49394900 & 0.44625200 & 0.54272500 \\
\hline $\mathrm{H}$ & 5.91630400 & 1.11961600 & 2.03924500 \\
\hline $\mathrm{O}$ & 5.40481400 & -0.66606000 & -1.56815800 \\
\hline $\mathrm{C}$ & 5.54774200 & -1.96431600 & 1.04376600 \\
\hline $\mathrm{O}$ & 5.97038300 & -1.93854600 & -0.29733600 \\
\hline $\mathrm{H}$ & 5.21960300 & -2.92512000 & 1.43355800 \\
\hline $\mathrm{C}$ & 5.00799300 & 2.69506700 & -0.19902600 \\
\hline $\mathrm{H}$ & 4.11640600 & 3.22161800 & -0.56438900 \\
\hline $\mathrm{C}$ & 5.93239800 & 2.48639500 & -1.40948800 \\
\hline $\mathrm{H}$ & 6.85170300 & 1.94497100 & -1.14555100 \\
\hline $\mathrm{H}$ & 5.43295500 & 1.97992400 & -2.24701700 \\
\hline
\end{tabular}




$\begin{array}{lrrr}\mathrm{H} & 6.26393600 & 3.44824000 & -1.81022700 \\ \mathrm{C} & 5.68116600 & 3.60618800 & 0.83895500 \\ \mathrm{H} & 5.88745900 & 4.58860500 & 0.40535400 \\ \mathrm{H} & 5.04057300 & 3.75781200 & 1.71353900 \\ \mathrm{H} & 6.63532400 & 3.19673900 & 1.18583700 \\ \mathrm{C} & -8.73844000 & 1.22839300 & -0.58761500 \\ \mathrm{H} & -9.27654600 & 1.94589100 & -1.20542300 \\ \mathrm{H} & -9.08821500 & 0.21482700 & -0.80419700 \\ \mathrm{H} & -8.93641800 & 1.42079600 & 0.47049500 \\ \mathrm{H} & 5.40598100 & -1.53572000 & -0.96700400 \\ \mathrm{H} & 5.35984800 & -0.46474200 & -2.52231400\end{array}$

\section{(Fig.11) output structure}

11

C $\quad-4.65388100 \quad-0.98108000 \quad 0.10507800$

$\begin{array}{llll}\text { C } & -3.08769700 & -0.91209100 & 0.02659900\end{array}$

$\begin{array}{llll}\text { C } & -2.37560300 & 0.23166200 & 0.82939100\end{array}$

$\begin{array}{llll}\mathrm{C} & -3.03771100 & 1.57423600 & 0.41287500\end{array}$

$\begin{array}{llll}\mathrm{C} & -4.56684100 & 1.56257200 & 0.51823700\end{array}$

$\mathrm{H} \quad-4.94442500 \quad 0.59162000 \quad-1.35096000$

$\mathrm{H} \quad-2.65471500 \quad 2.39649200 \quad 1.02580000$

$\mathrm{H} \quad-2.76289800 \quad 1.80563100 \quad-0.62537600$

$\mathrm{H} \quad-4.89428000 \quad 1.48456200 \quad 1.56031400$

$\mathrm{H} \quad-4.96277700 \quad 2.50742900 \quad 0.13530200$

$\mathrm{H} \quad-2.89755000 \quad-0.65355800 \quad-1.02832500$

$\begin{array}{llll}\mathrm{C} & -2.49366800 & 0.10520600 & 2.36948900\end{array}$

$\mathrm{H} \quad-2.53515400 \quad-0.92621200 \quad 2.72223800$

$\mathrm{H} \quad-1.65533600 \quad 0.59328500 \quad 2.87295100$

$\begin{array}{llll}\mathrm{H} & -3.39705000 & 0.59425800 & 2.73664600\end{array}$

$\begin{array}{llll}\mathrm{C} & -0.87392000 & 0.25947600 & 0.32879600\end{array}$

$\mathrm{H} \quad-0.98866300 \quad 0.48331300 \quad-0.73853200$

C $\quad-2.38051200 \quad-2.25656300 \quad 0.24988300$

$\mathrm{H} \quad-2.89947900 \quad-3.05254000 \quad-0.29001000$

$\mathrm{H} \quad-2.40837200 \quad-2.54658000 \quad 1.30525200$

C $\quad-0.93535800 \quad-2.20580600 \quad-0.26524900$

$\mathrm{H} \quad-0.98609800 \quad-2.05038100 \quad-1.34745100$

$\mathrm{H} \quad-0.46333200 \quad-3.18388400 \quad-0.11347600$

$\begin{array}{llll}\mathrm{C} & -0.07280200 & -1.09542900 & 0.38791800\end{array}$

C $\quad-5.17323300 \quad-1.97595700 \quad-0.95821700$

$\mathrm{H} \quad-4.99299300 \quad-3.01544000 \quad-0.67212400$

$\mathrm{H} \quad-6.25302400 \quad-1.85711700 \quad-1.07726200$

$\mathrm{H} \quad-4.70598600 \quad-1.80401600 \quad-1.93449400$

C $\quad-5.20955400 \quad-1.42956400 \quad 1.47201800$

$\mathrm{H} \quad-5.03291500 \quad-0.70939800 \quad 2.27138300$

$\mathrm{H} \quad-6.28973700 \quad-1.57612000 \quad 1.40094800$

$\mathrm{H} \quad-4.76644800 \quad-2.38273700 \quad 1.77596500$

O $\quad-6.60933700 \quad 0.43236500 \quad-0.15201800$

$\begin{array}{llll}\mathrm{C} & -7.29803700 & 1.25331800 & -0.98179300\end{array}$

$\begin{array}{llll}\mathrm{O} & -6.77757400 & 1.96062800 & -1.80892800\end{array}$

C $\quad 0.22011800 \quad-1.53482700 \quad 1.84365600$

$\mathrm{H} \quad 0.72557200 \quad-2.50363300 \quad 1.87151000$

$\mathrm{H} \quad 0.82882700 \quad-0.82341400 \quad 2.40621000$

$\mathrm{H} \quad-0.70087200 \quad-1.66252800 \quad 2.40617400$

$\begin{array}{llll}\mathrm{C} & 1.30689300 & -0.91609600 & -0.42324900\end{array}$

$\begin{array}{llll}\mathrm{C} & -0.04017200 & 1.41981600 & 0.89997000\end{array}$

$\begin{array}{llll}\mathrm{H} & -0.59337500 & 2.35814600 & 0.82001300\end{array}$

$\begin{array}{llll}\mathrm{H} & 0.16021200 & 1.27593500 & 1.96775200\end{array}$

$\begin{array}{llll}\mathrm{C} & 2.11083700 & 0.27984300 & 0.17897800\end{array}$

$\begin{array}{llll}\mathrm{C} & 1.29292100 & 1.57848200 & 0.15995600\end{array}$

$\mathrm{H} \quad 1.86190800 \quad 2.39644100 \quad 0.61543100$

$\begin{array}{llll}\mathrm{H} & 1.10925500 & 1.87906100 & -0.87988900\end{array}$

C $\quad \begin{array}{llll}1.04375200 & -0.69864700 & -1.93774500\end{array}$ 


$\begin{array}{lrrr}\mathrm{H} & 1.90305000 & -0.25330200 & -2.44669200 \\ \mathrm{H} & 0.83225400 & -1.64501000 & -2.44277600 \\ \mathrm{H} & 0.20239900 & -0.03532300 & -2.13412600 \\ \mathrm{H} & 2.28673200 & 0.03042100 & 1.23423600 \\ \mathrm{C} & 3.52396100 & 0.50740600 & -0.44227900 \\ \mathrm{H} & 3.40539100 & 0.96220100 & -1.43065000 \\ \mathrm{C} & 2.20424100 & -2.17126700 & -0.27366800 \\ \mathrm{H} & 2.41636600 & -2.36029900 & 0.78310300 \\ \mathrm{H} & 1.69214800 & -3.06199300 & -0.64703100 \\ \mathrm{C} & 3.53263900 & -2.03514600 & -1.02718100 \\ \mathrm{H} & 3.32954700 & -1.96309100 & -2.10246300 \\ \mathrm{C} & 4.32883100 & -0.79912600 & -0.61587600 \\ \mathrm{H} & 4.14288200 & -2.93754300 & -0.89750900 \\ \mathrm{C} & 5.12633600 & -0.91438800 & 0.70703000 \\ \mathrm{H} & 4.47301900 & -1.24075800 & 1.51960700 \\ \mathrm{C} & 4.39705100 & 1.42661800 & 0.47845700 \\ \mathrm{H} & 3.79317900 & 1.66126300 & 1.36066000 \\ \mathrm{C} & 5.59173300 & 0.56664400 & 0.98443300 \\ \mathrm{H} & 6.50004800 & 0.77706800 & 0.41516400 \\ \mathrm{H} & 5.81362000 & 0.72156600 & 2.04087400 \\ \mathrm{O} & 5.40670800 & -0.58602300 & -1.62032900 \\ \mathrm{C} & 6.31677100 & -1.77564100 & 0.65630800 \\ \mathrm{O} & 6.9581100 & -1.97197000 & -0.40346500 \\ \mathrm{H} & 6.73193800 & -2.24179900 & 1.55450100 \\ \mathrm{C} & 4.82011400 & 2.79292400 & -0.12106300 \\ \mathrm{H} & 3.88007000 & 3.27702000 & -0.41810800 \\ \mathrm{C} & 5.70475300 & 2.71360600 & -1.37283100 \\ \mathrm{H} & 6.69285900 & 2.29226500 & -1.15693400 \\ \mathrm{H} & 5.25403100 & 2.11716200 & -2.17038800 \\ \mathrm{H} & 5.87011200 & 3.71904200 & -1.77004300 \\ \mathrm{C} & 5.47391300 & 3.67957400 & 0.94947800 \\ \mathrm{H} & 5.66384900 & 4.68188100 & 0.55579400 \\ \mathrm{H} & 4.83494400 & 3.78438200 & 1.83242000 \\ \mathrm{H} & 6.43823200 & 3.27449000 & 1.27785300 \\ \mathrm{C} & -8.77998900 & 1.15003500 & -0.72465500 \\ \mathrm{H} & -9.31017300 & 1.83998300 & -1.37959900 \\ \mathrm{H} & -9.12186400 & 0.12708500 & -0.90713800 \\ \mathrm{H} & -8.99704500 & 1.38302400 & 0.32147000 \\ \mathrm{H} & 6.42094500 & -1.44042200 & -1.17331000 \\ \mathrm{H} & 5.04633100 & -0.69948500 & -2.51033500 \\ & & & \\ & & & \end{array}$




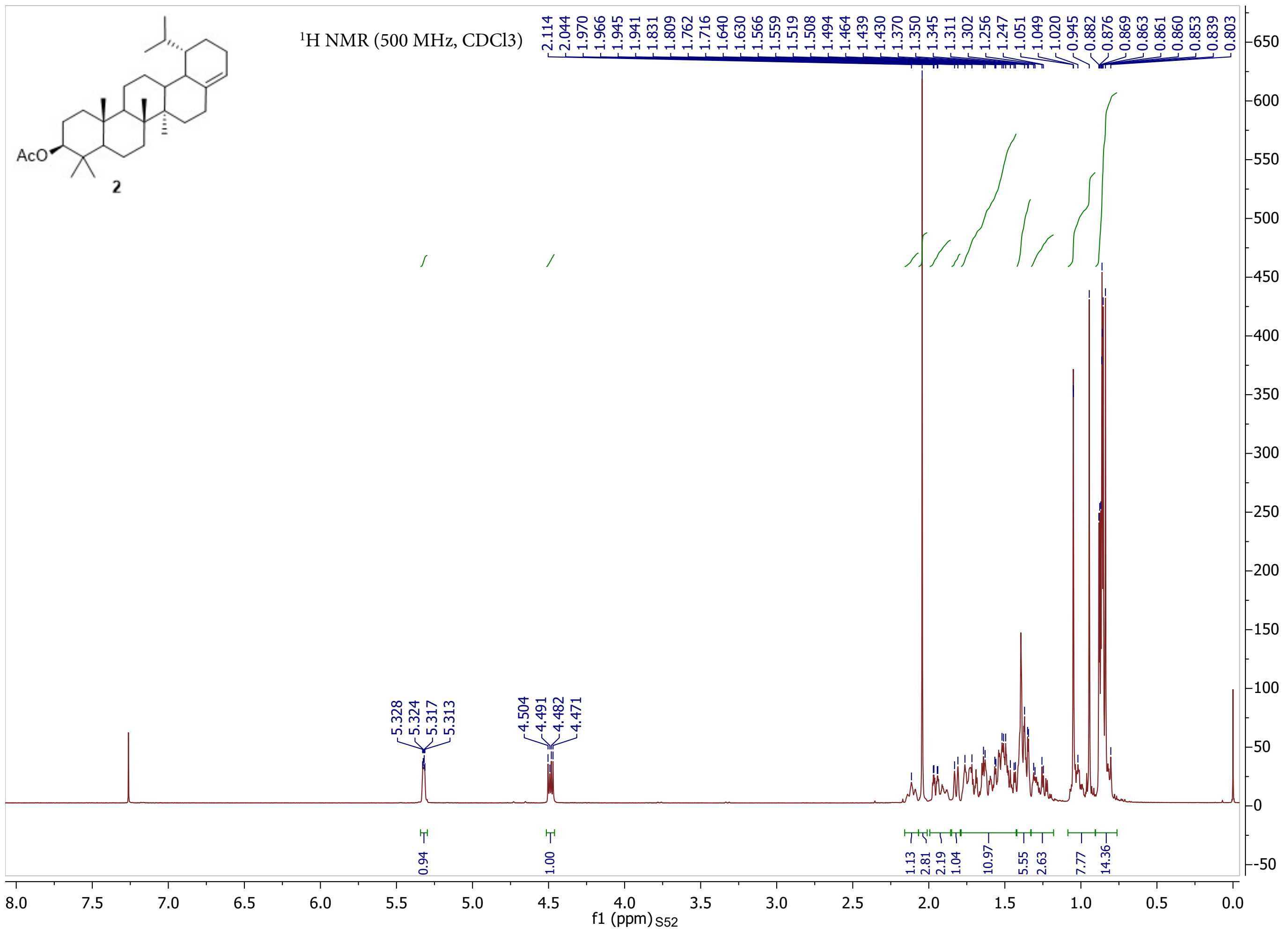




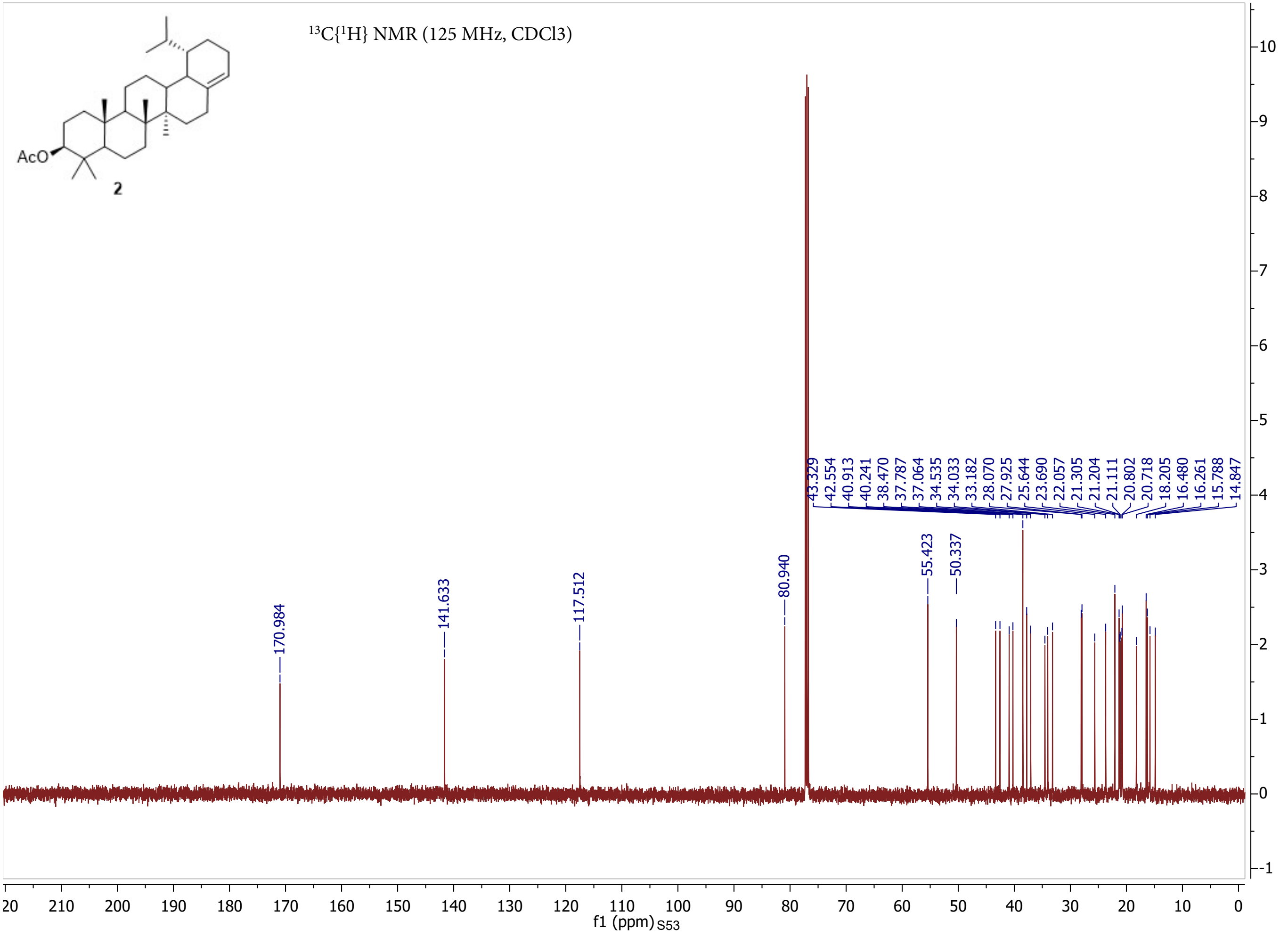




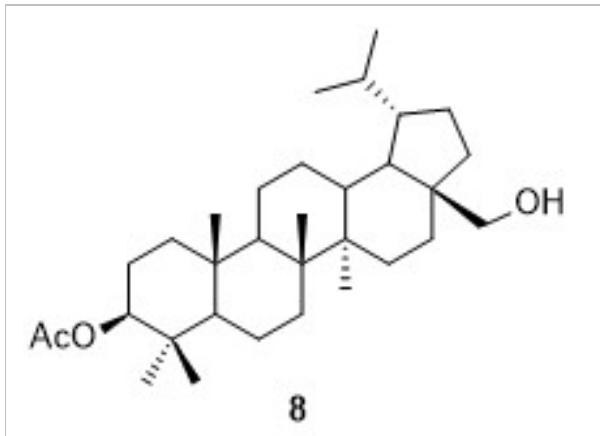

${ }^{1} \mathrm{H}$ NMR $(500 \mathrm{MHz}, \mathrm{CDCl} 3)$

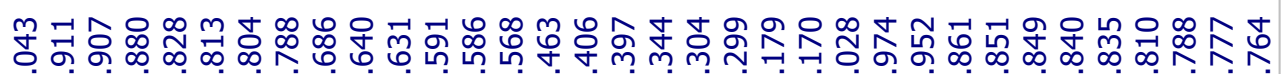
نं
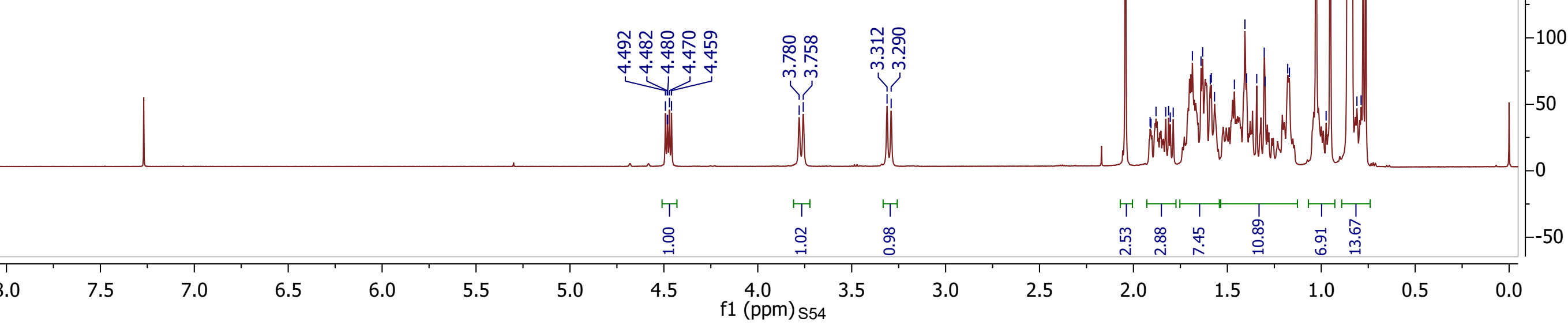


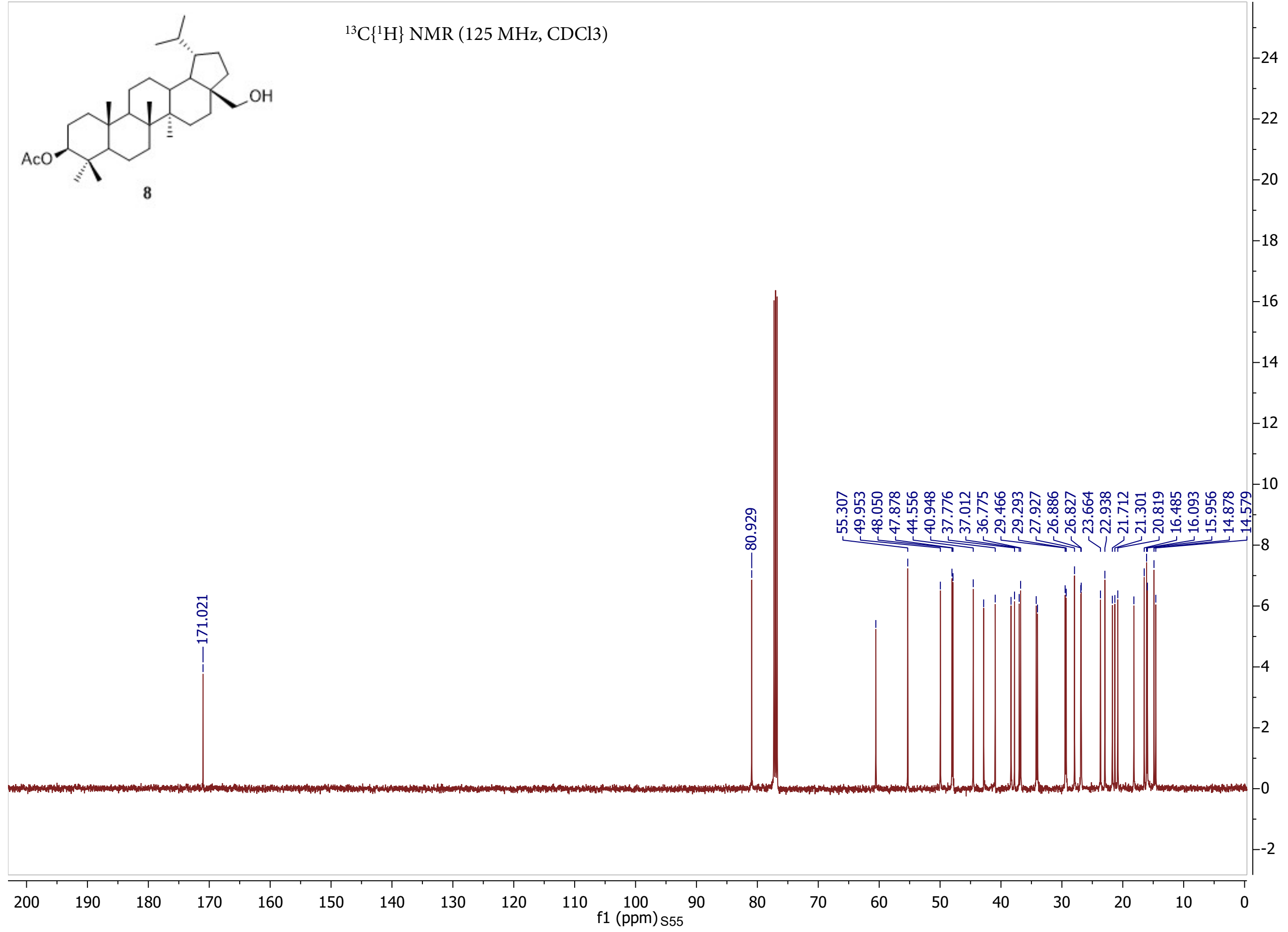




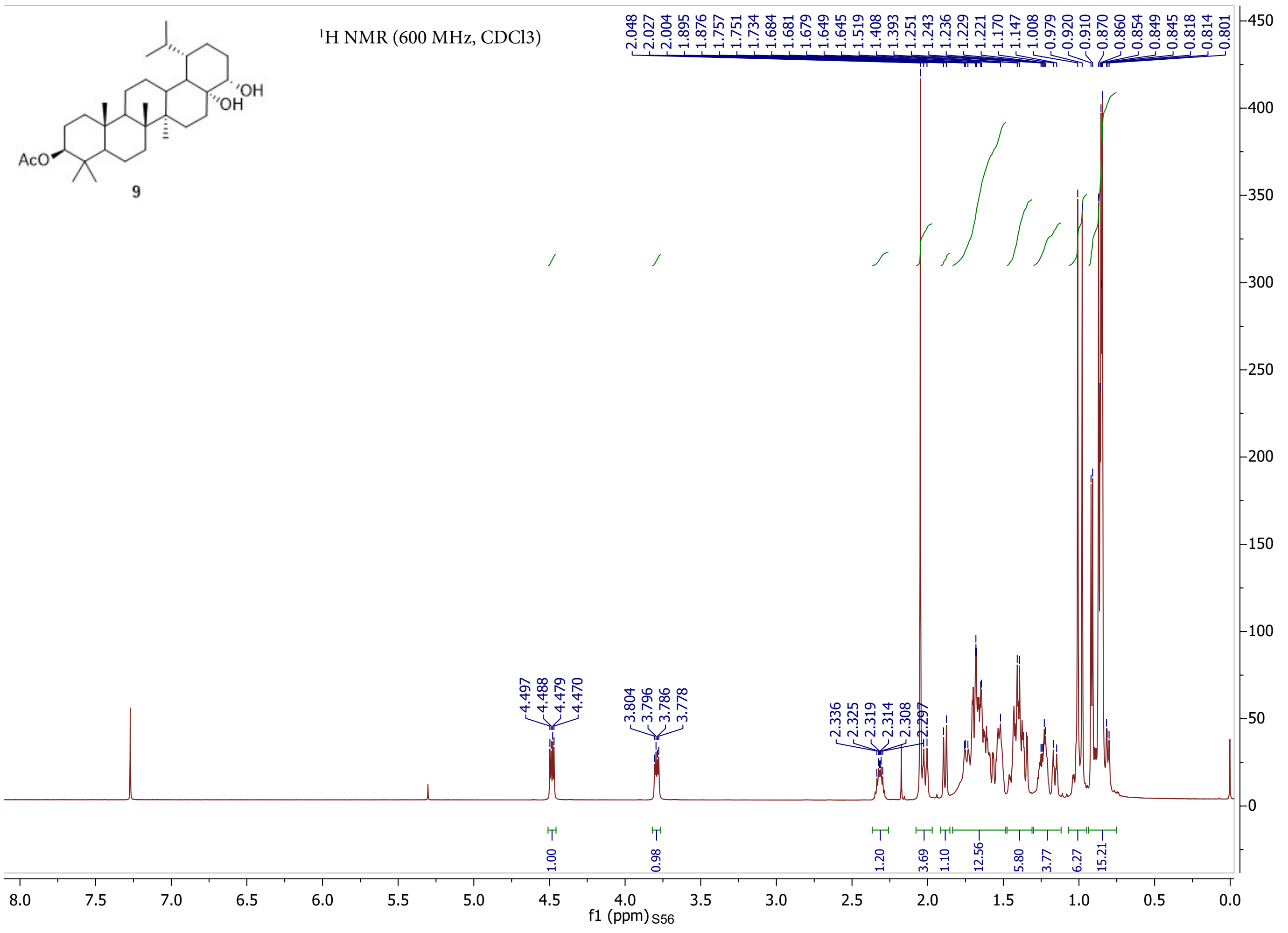




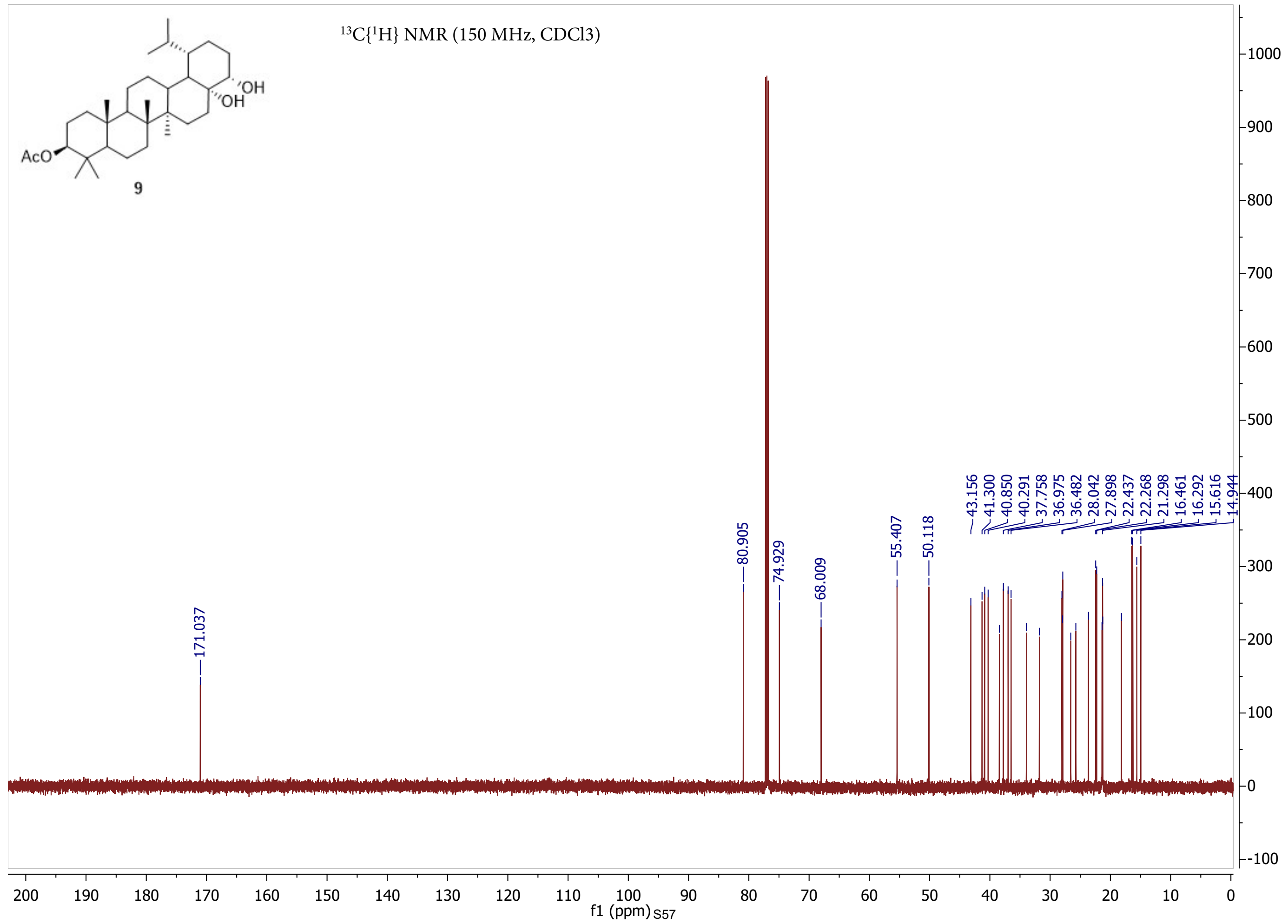




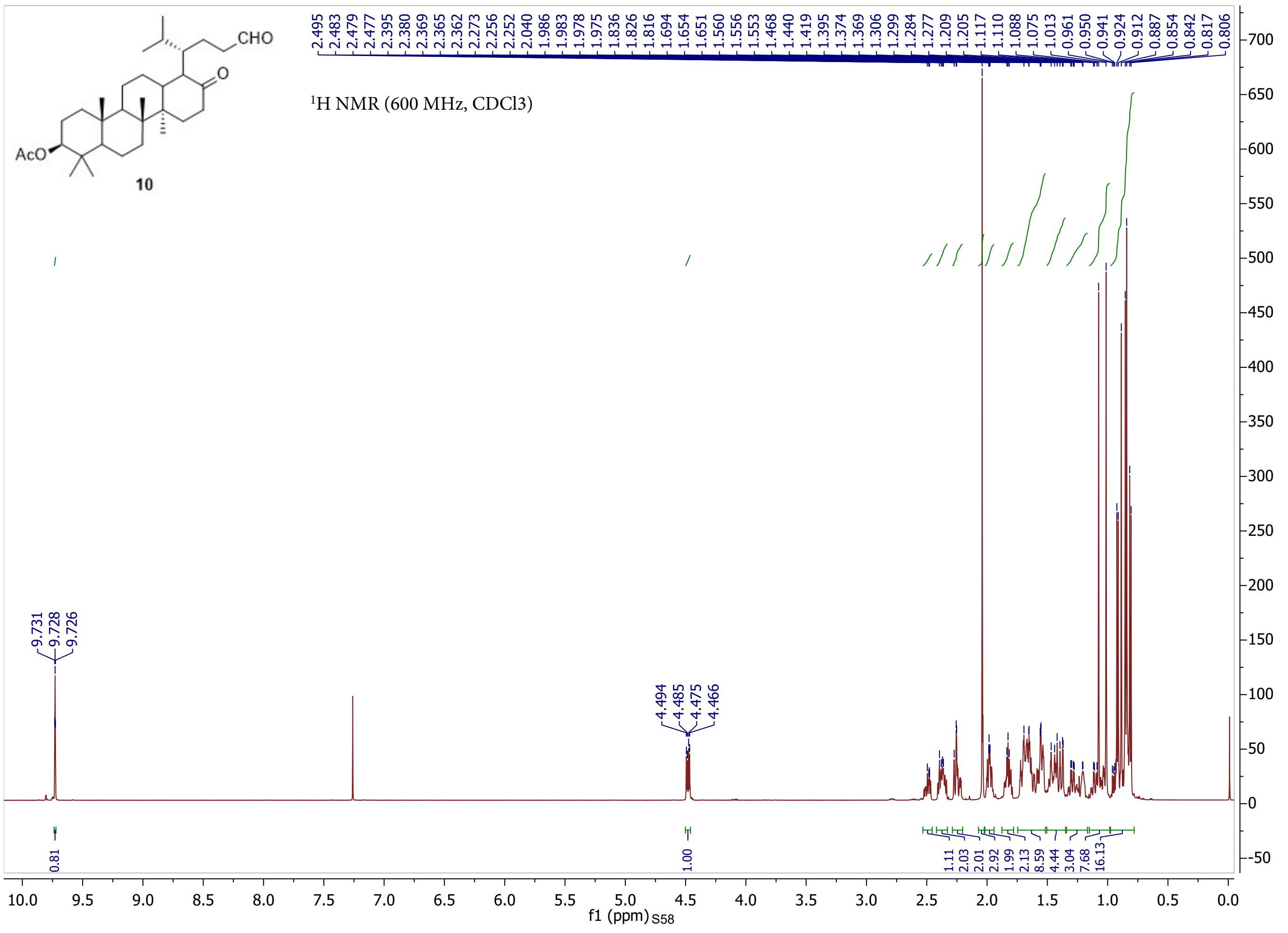




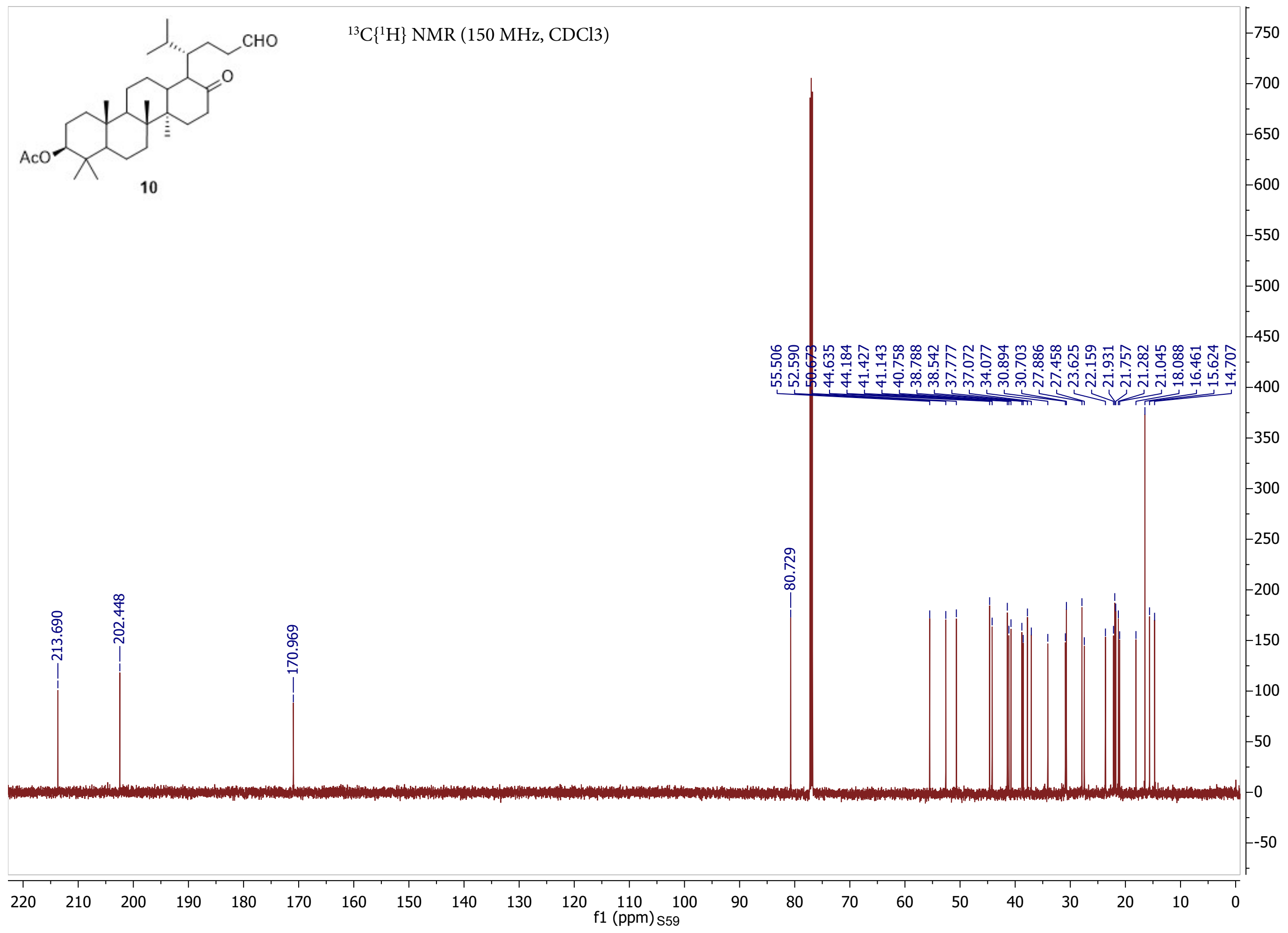




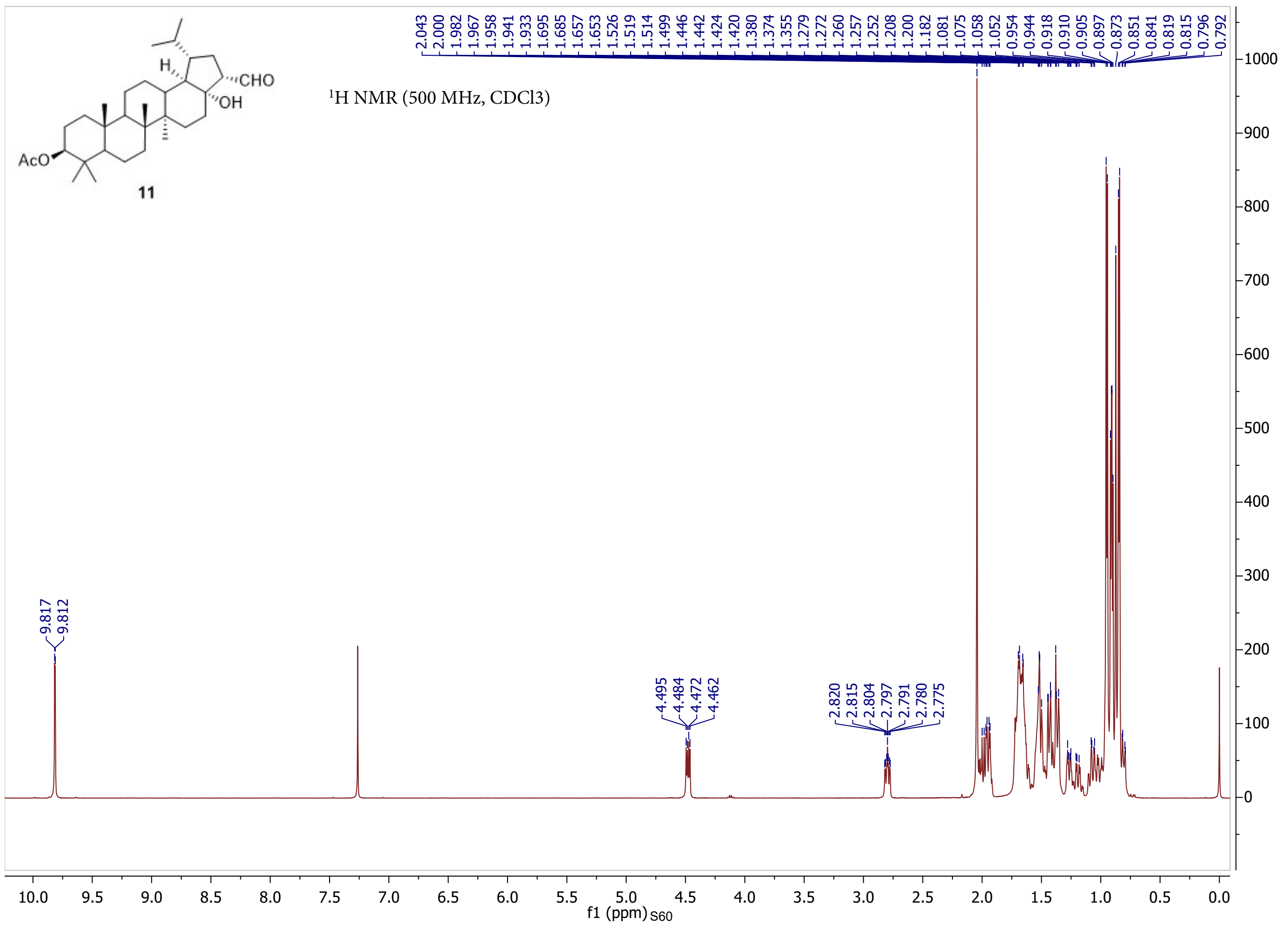




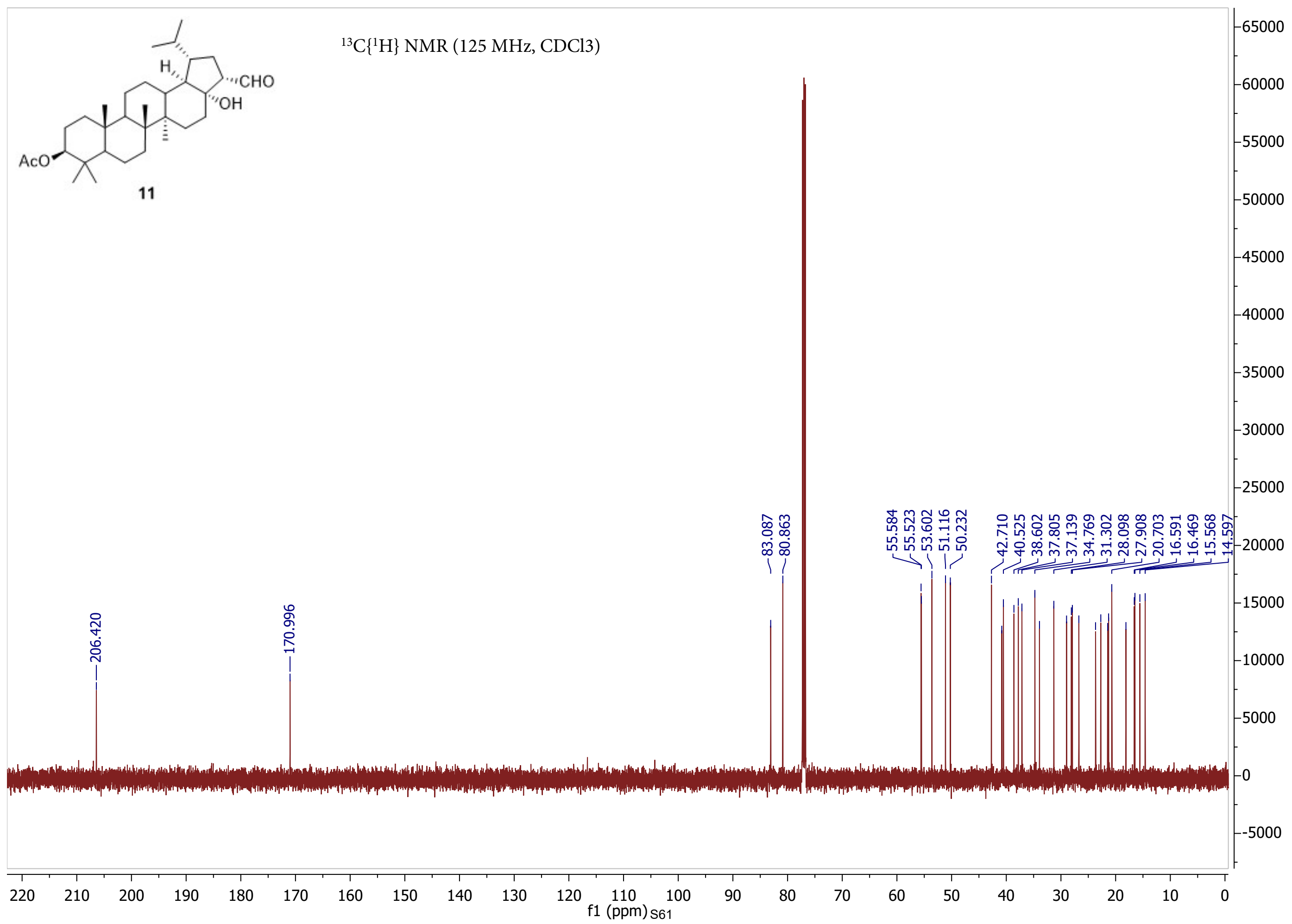




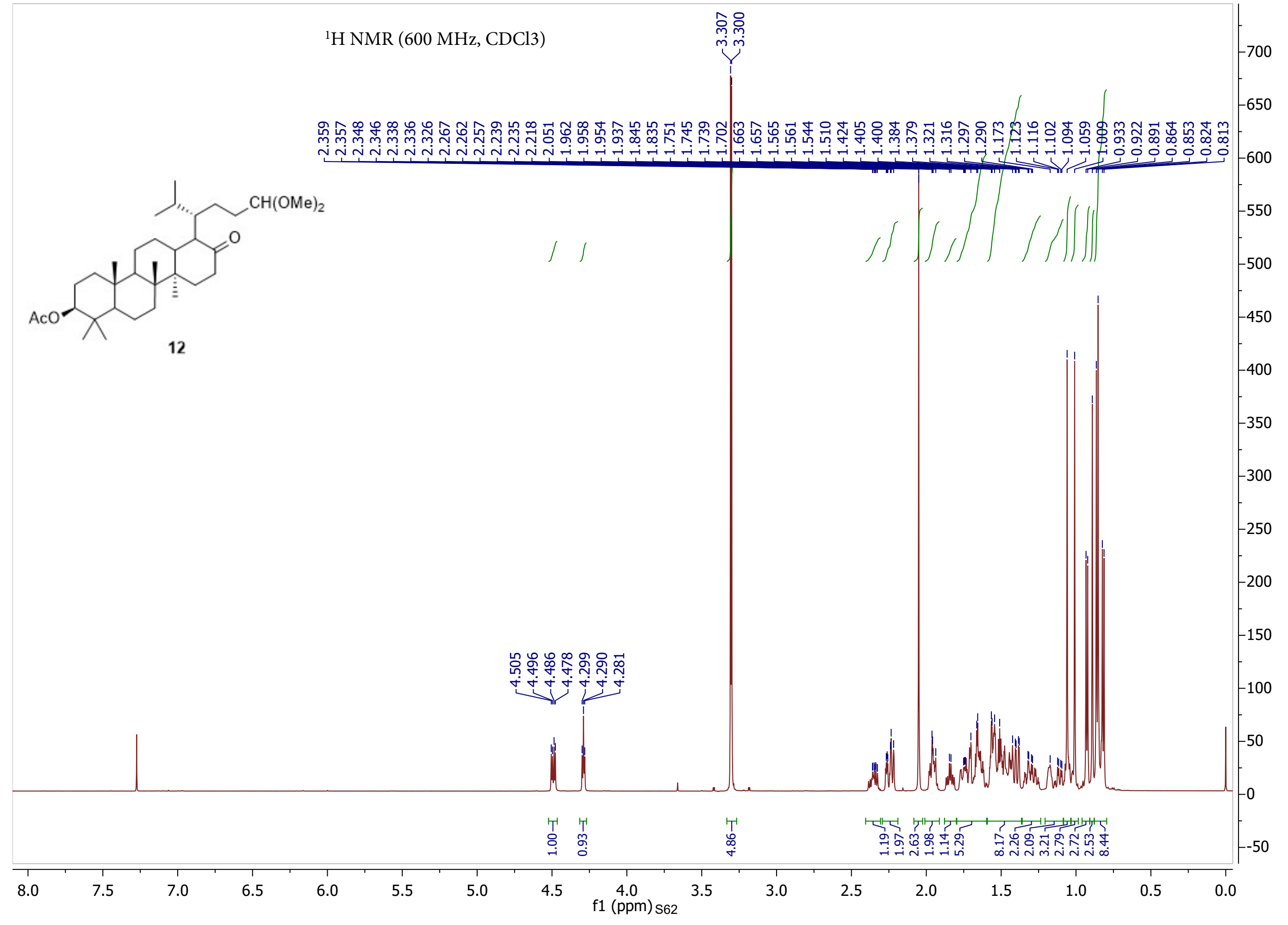




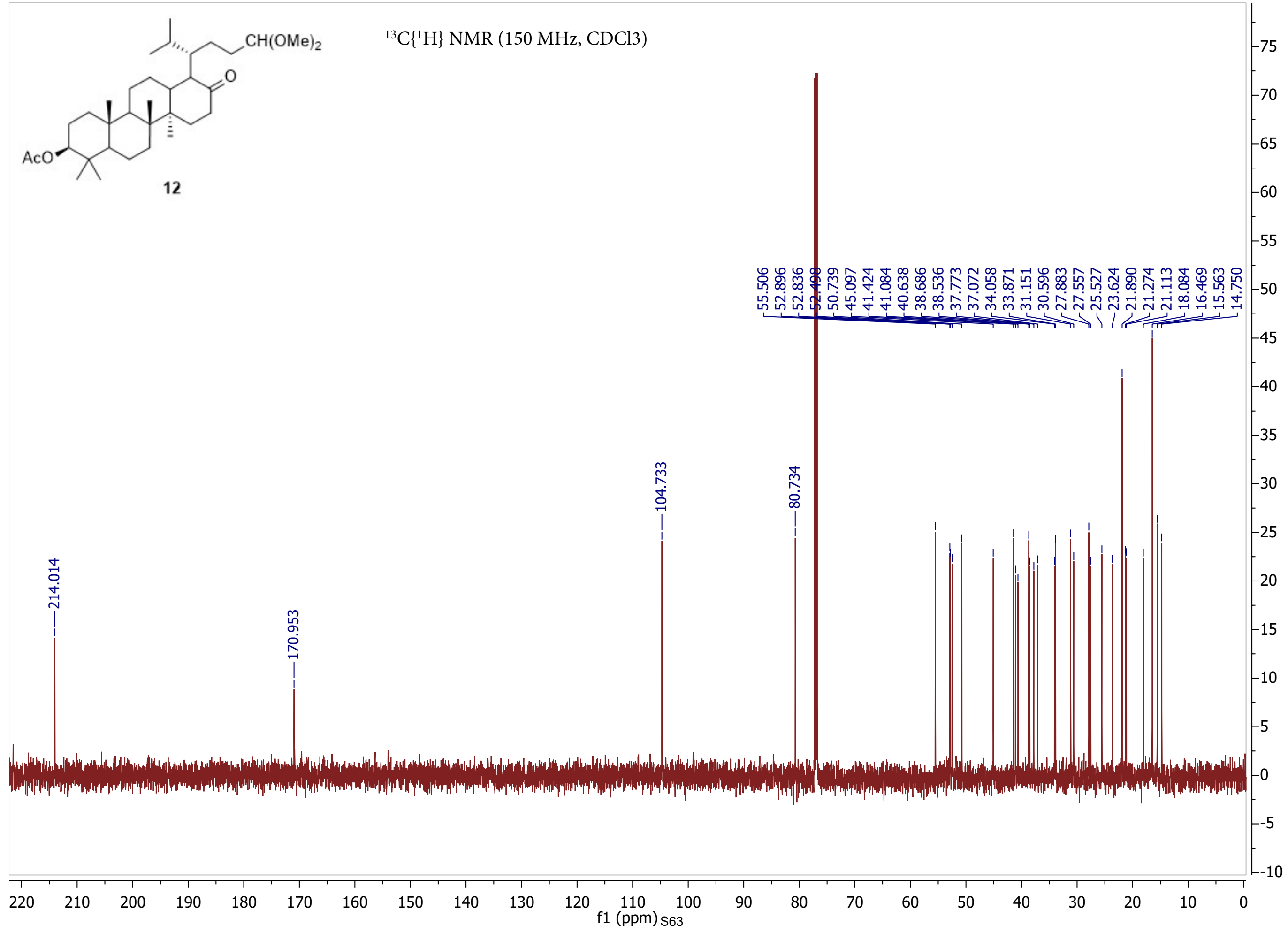




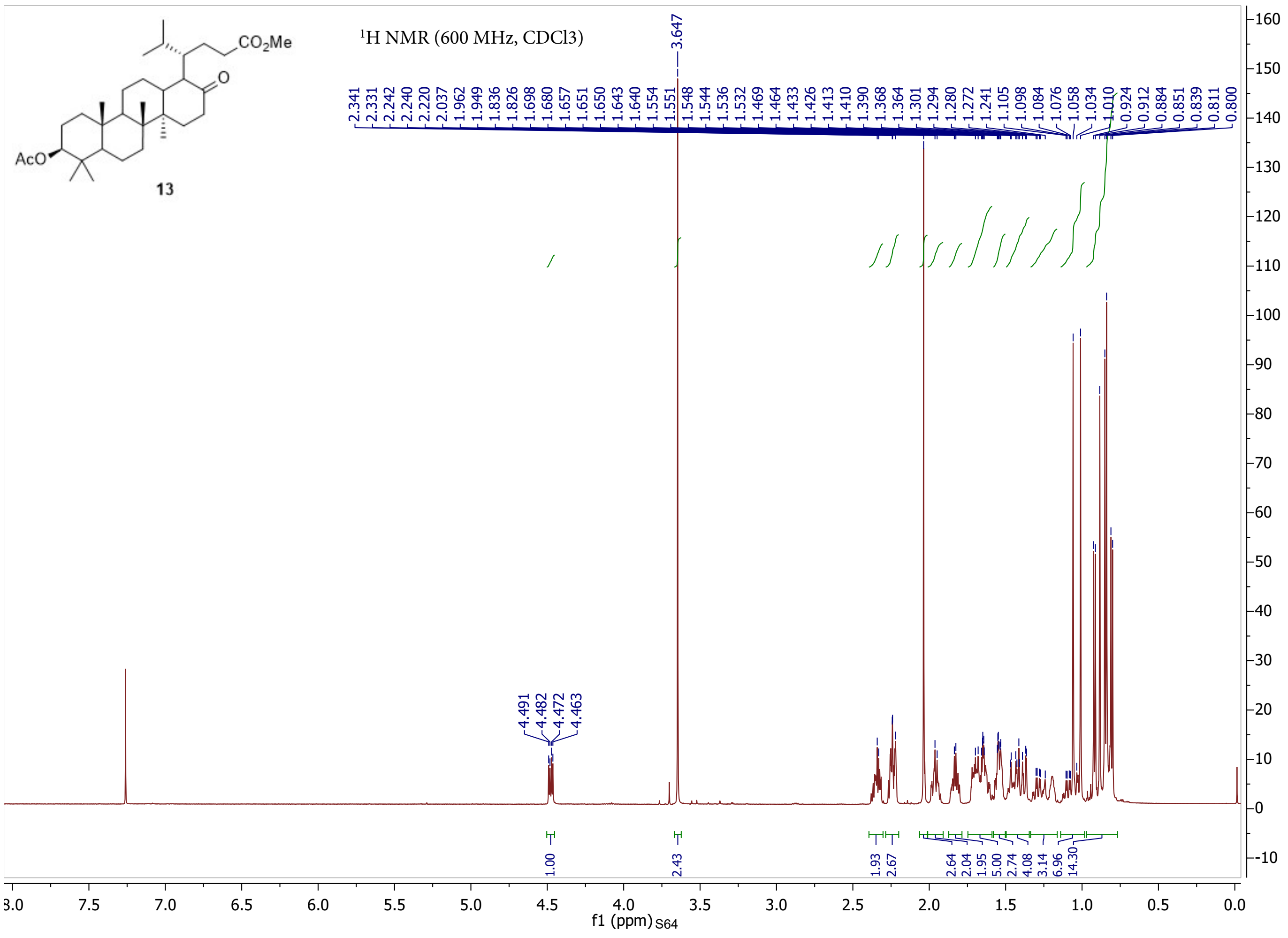




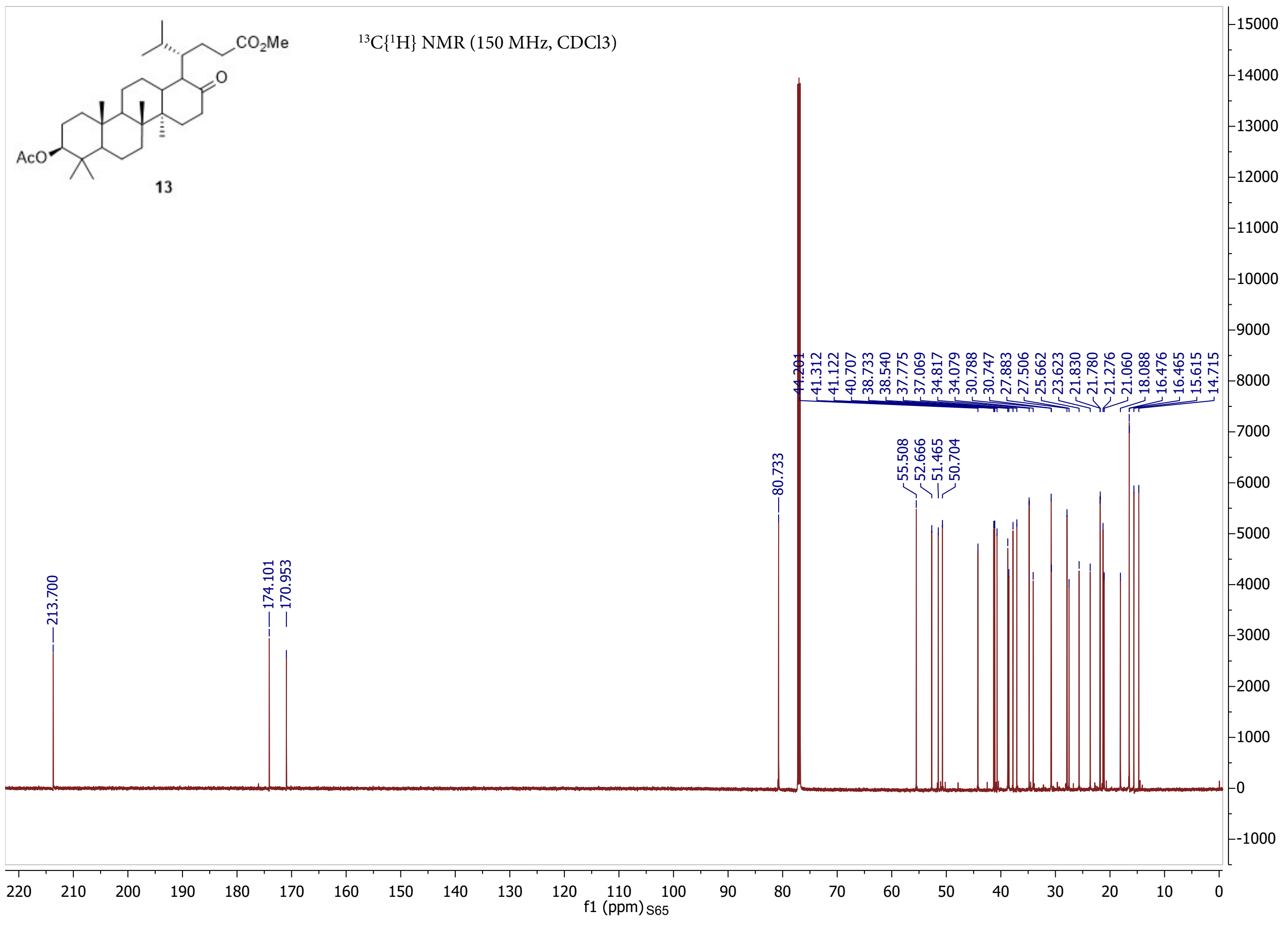




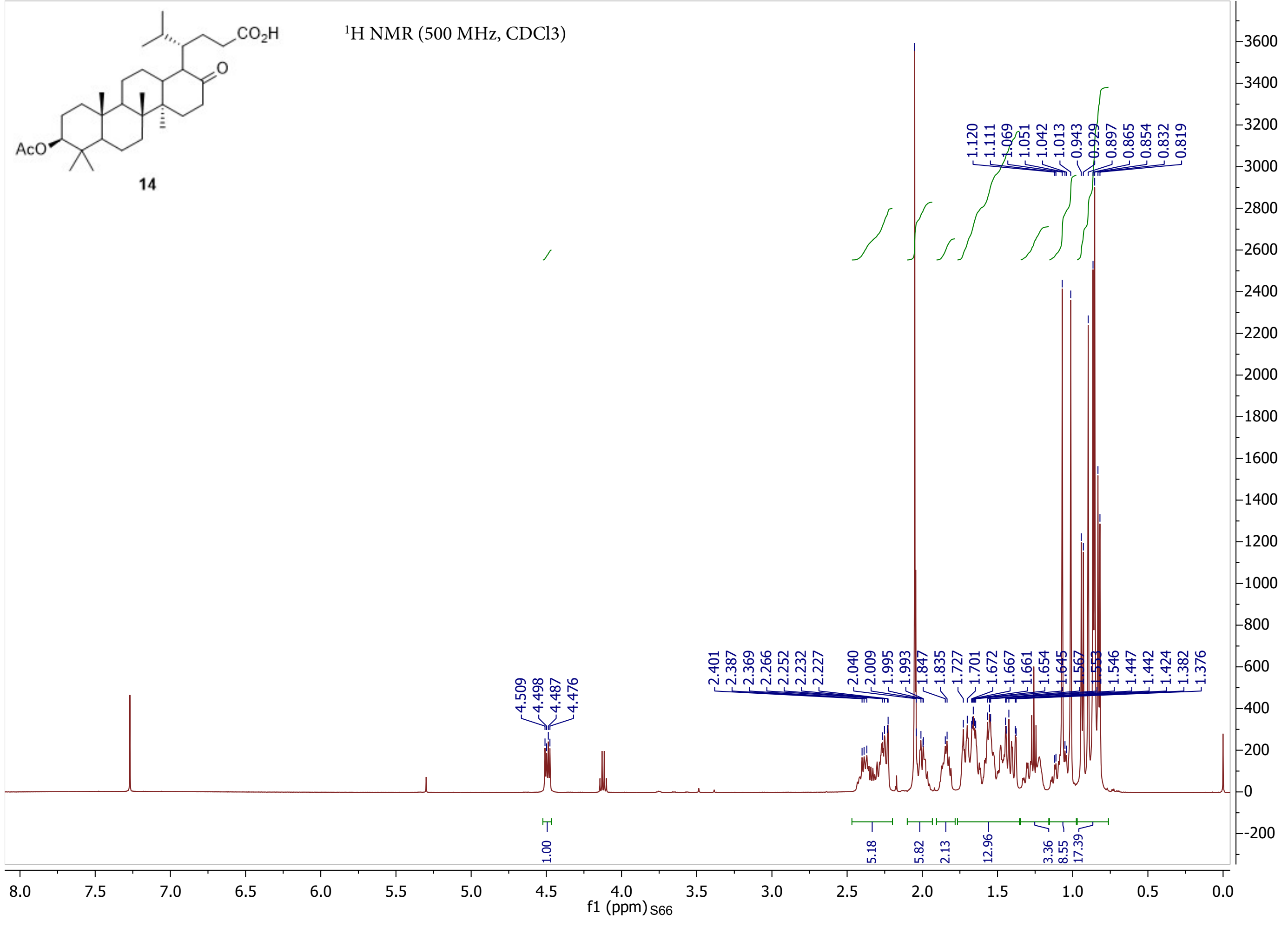




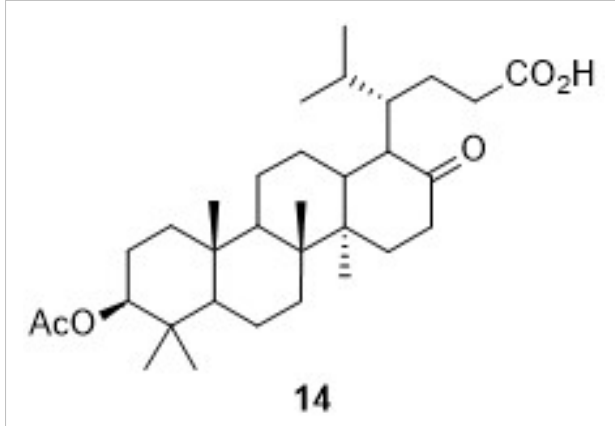




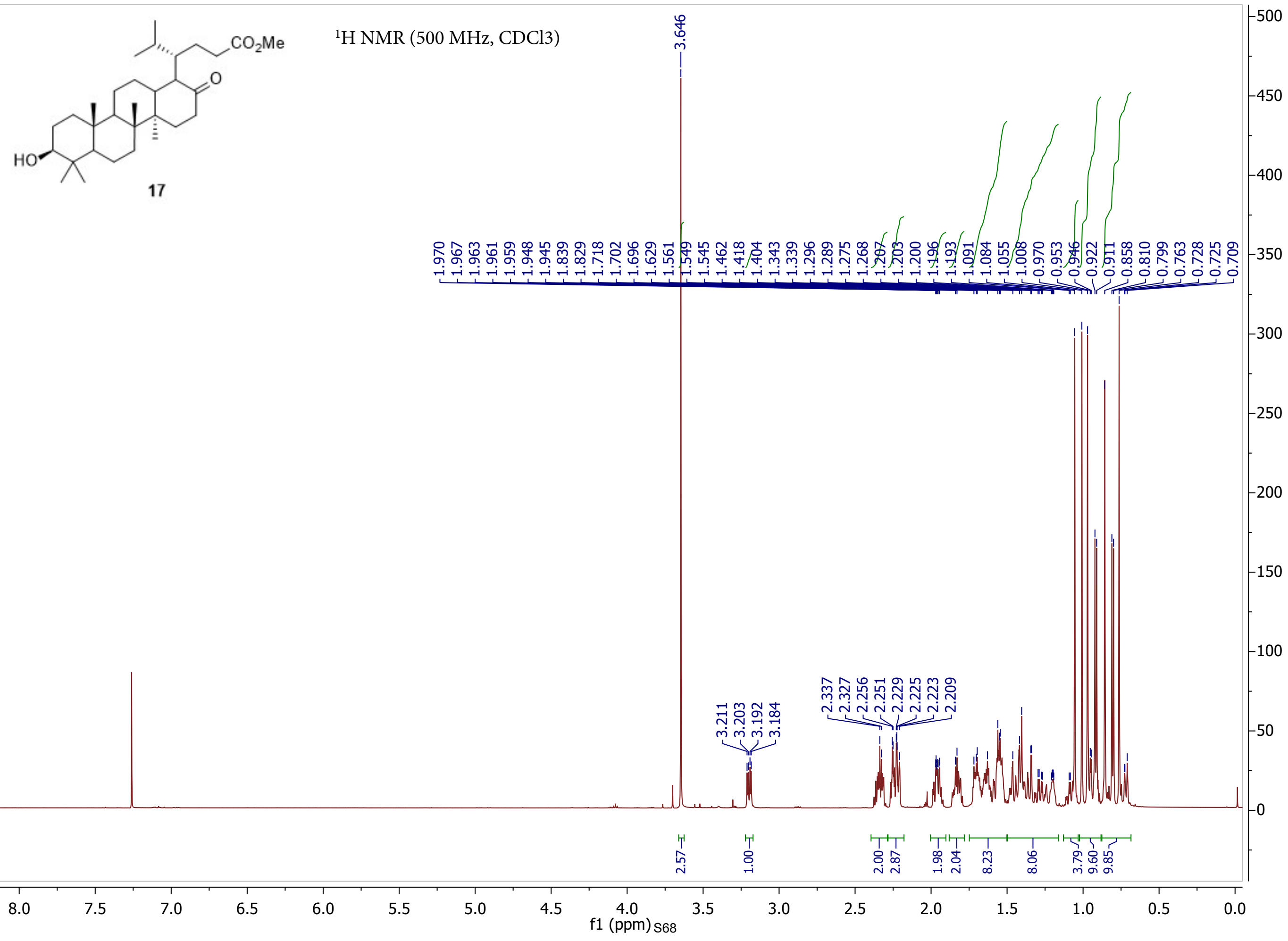




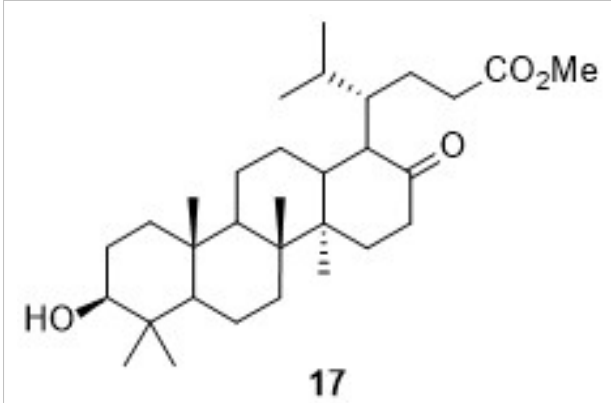

${ }^{13} \mathrm{C}\left\{{ }^{1} \mathrm{H}\right\}$ NMR (125 MHz, CDCl3)

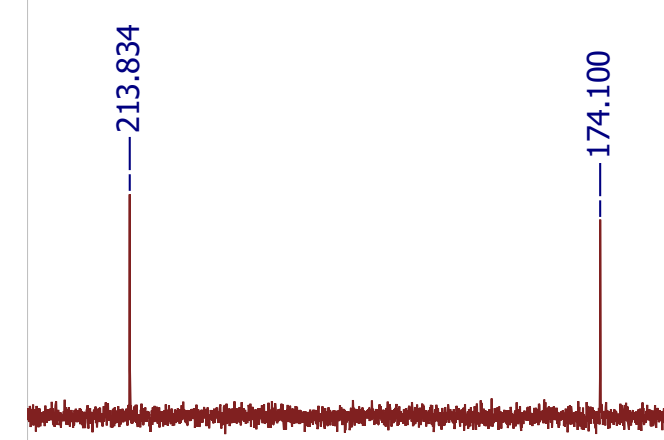




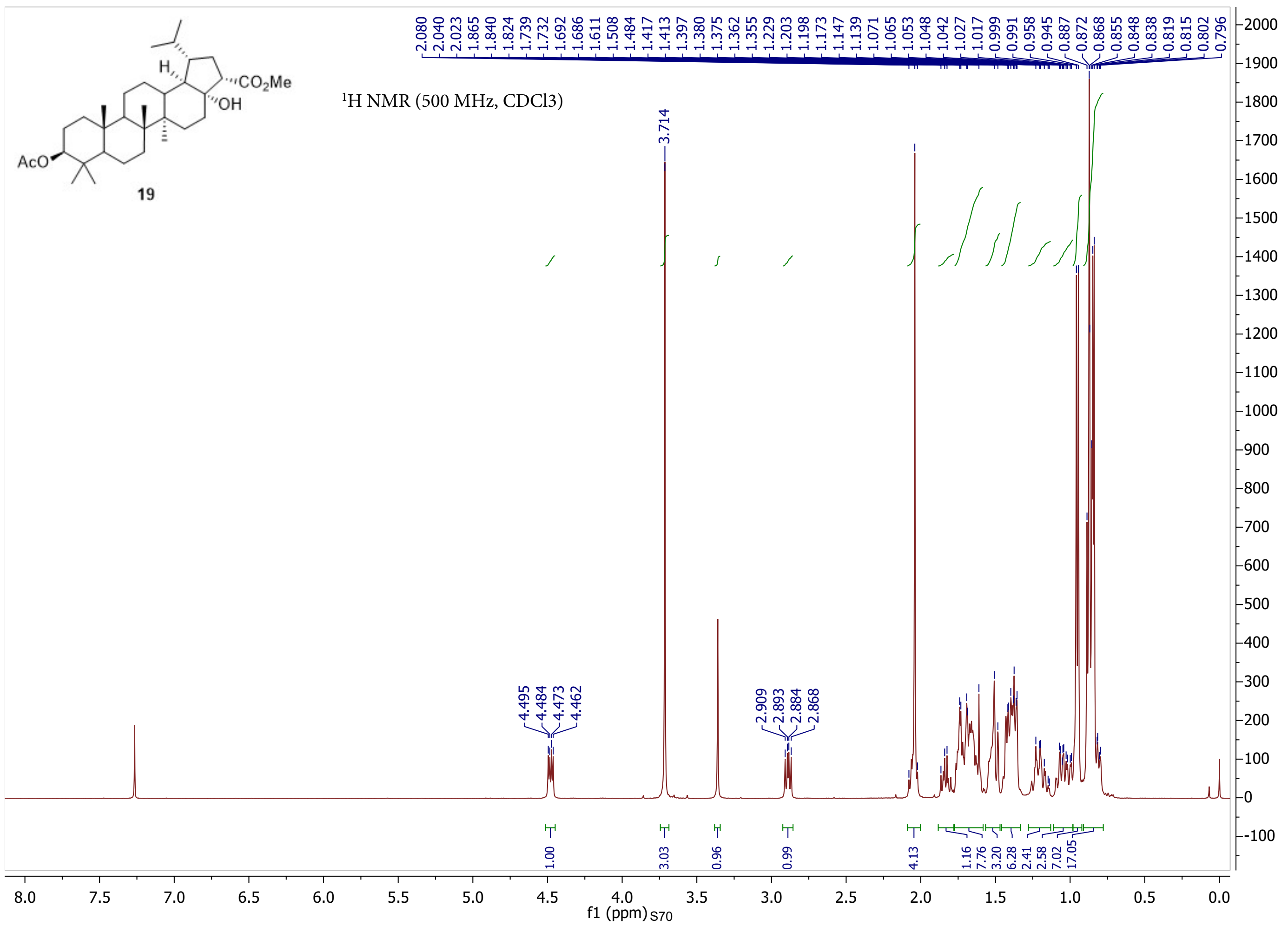




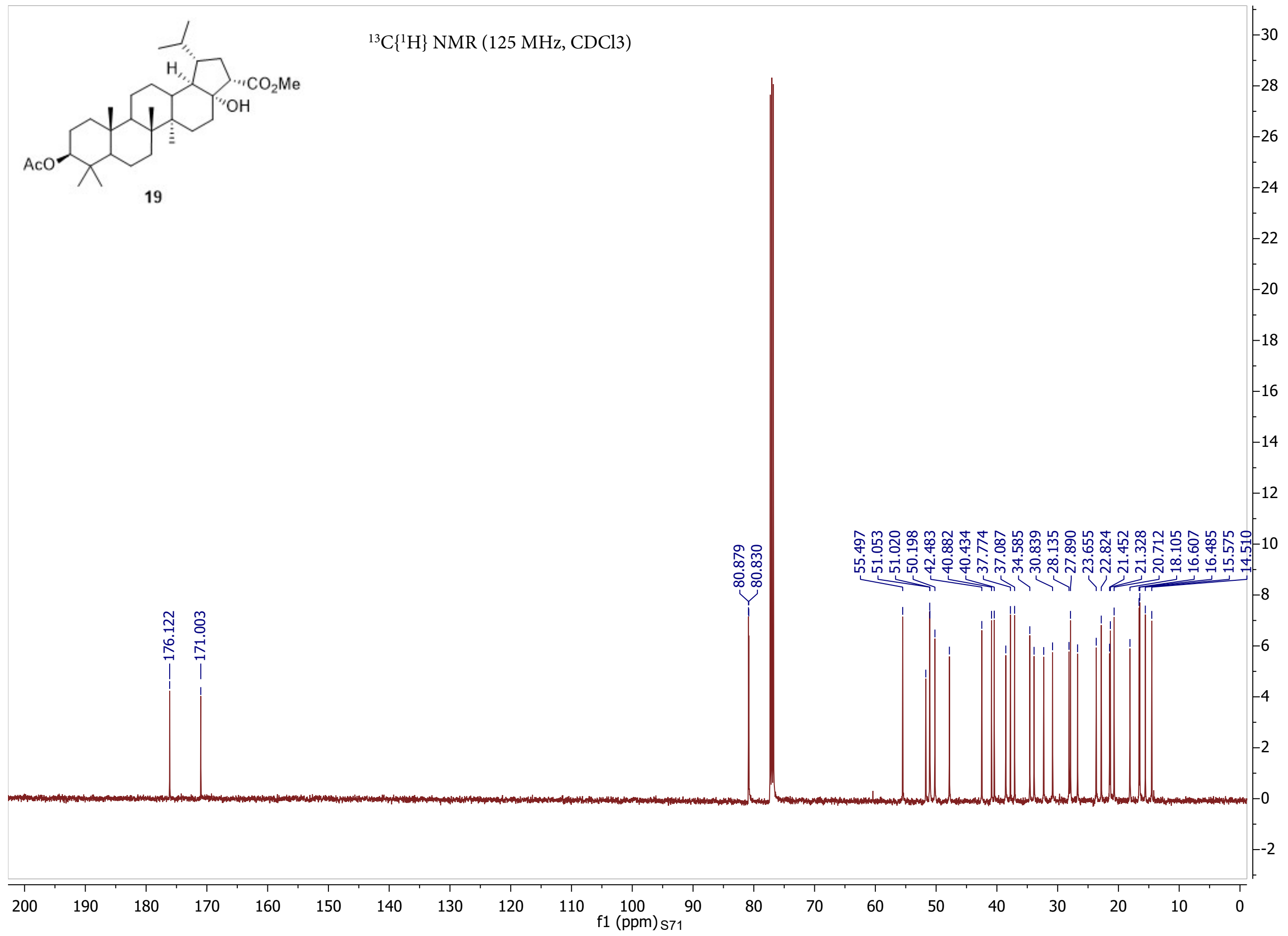




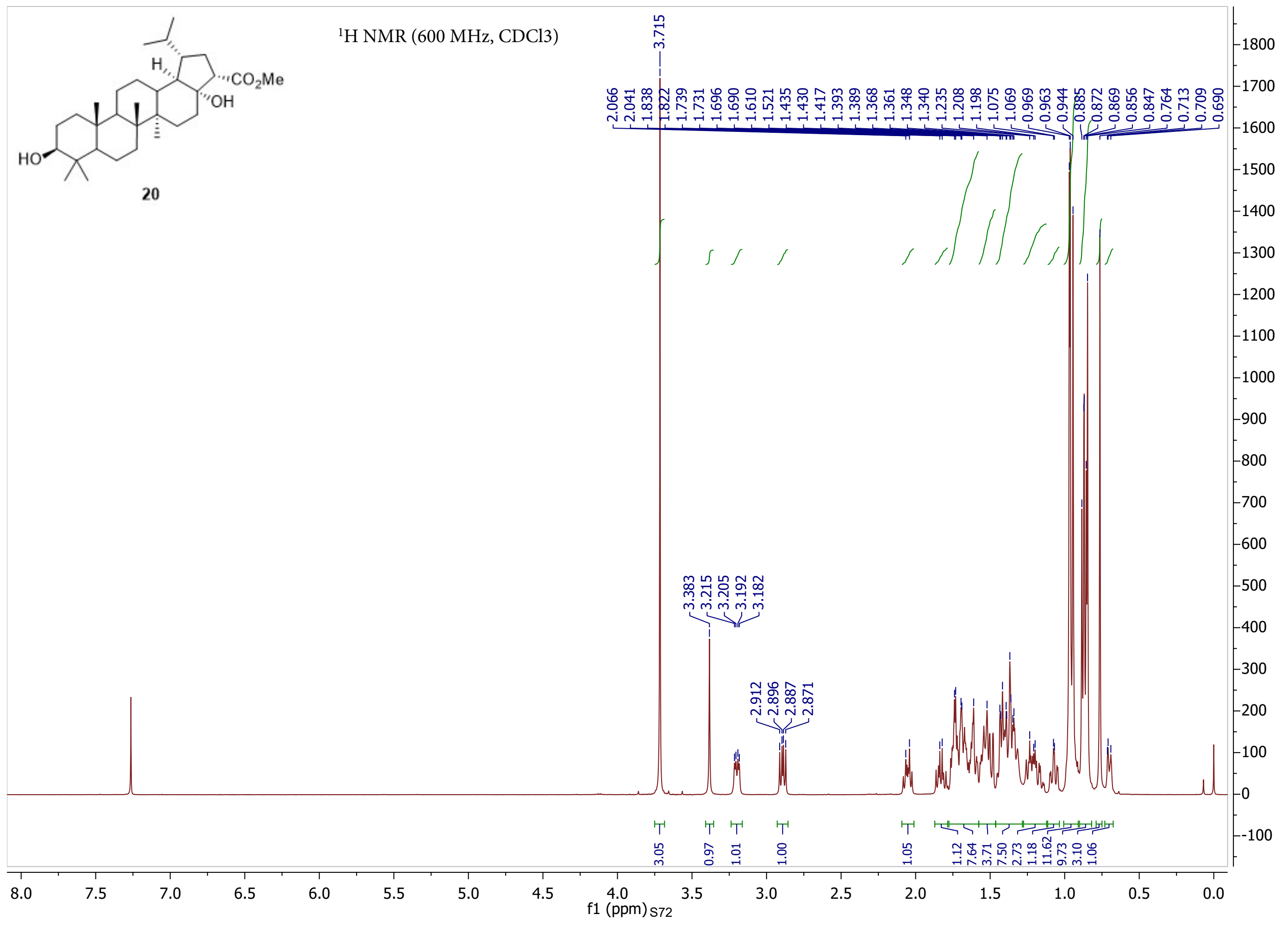




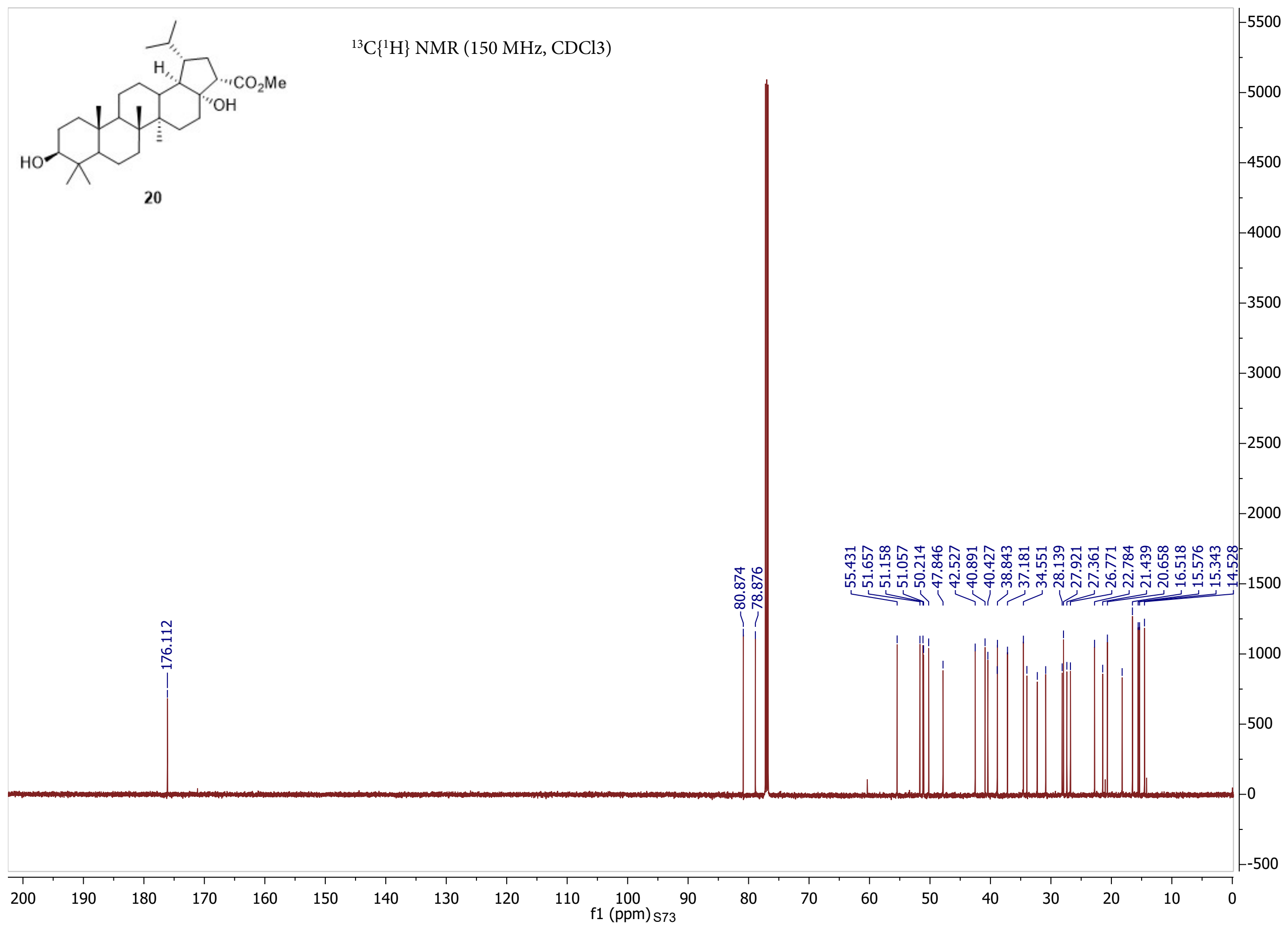




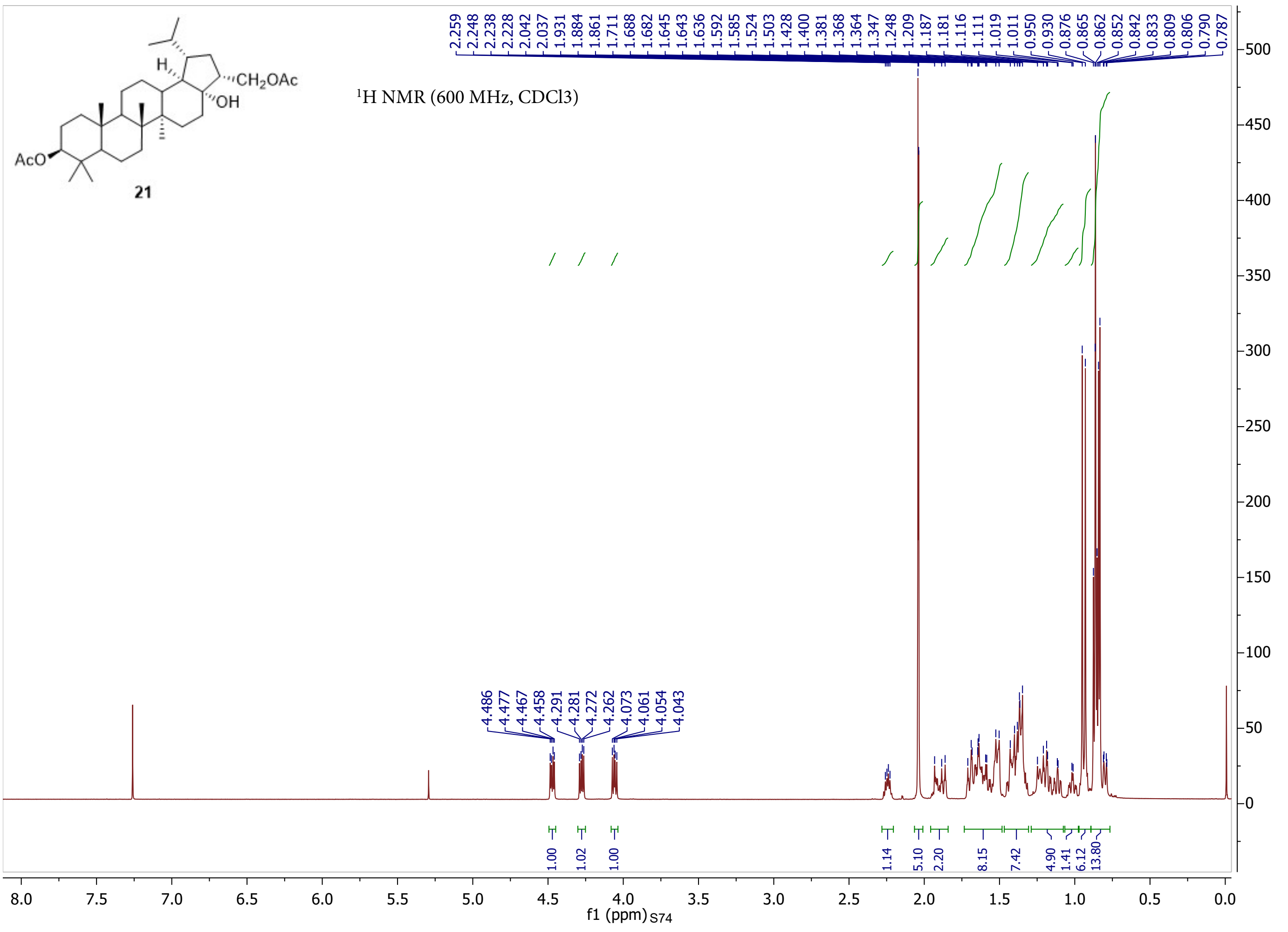




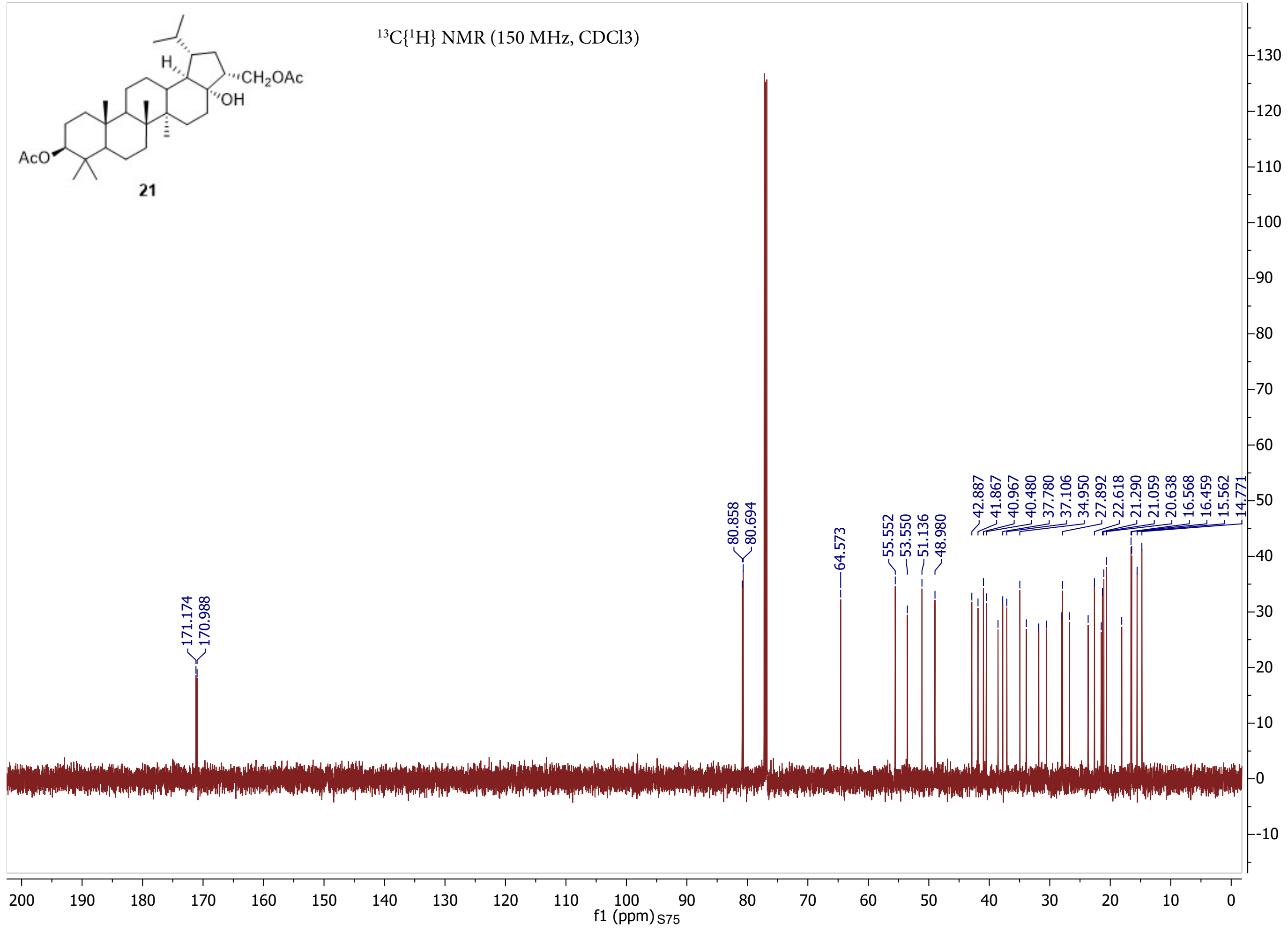




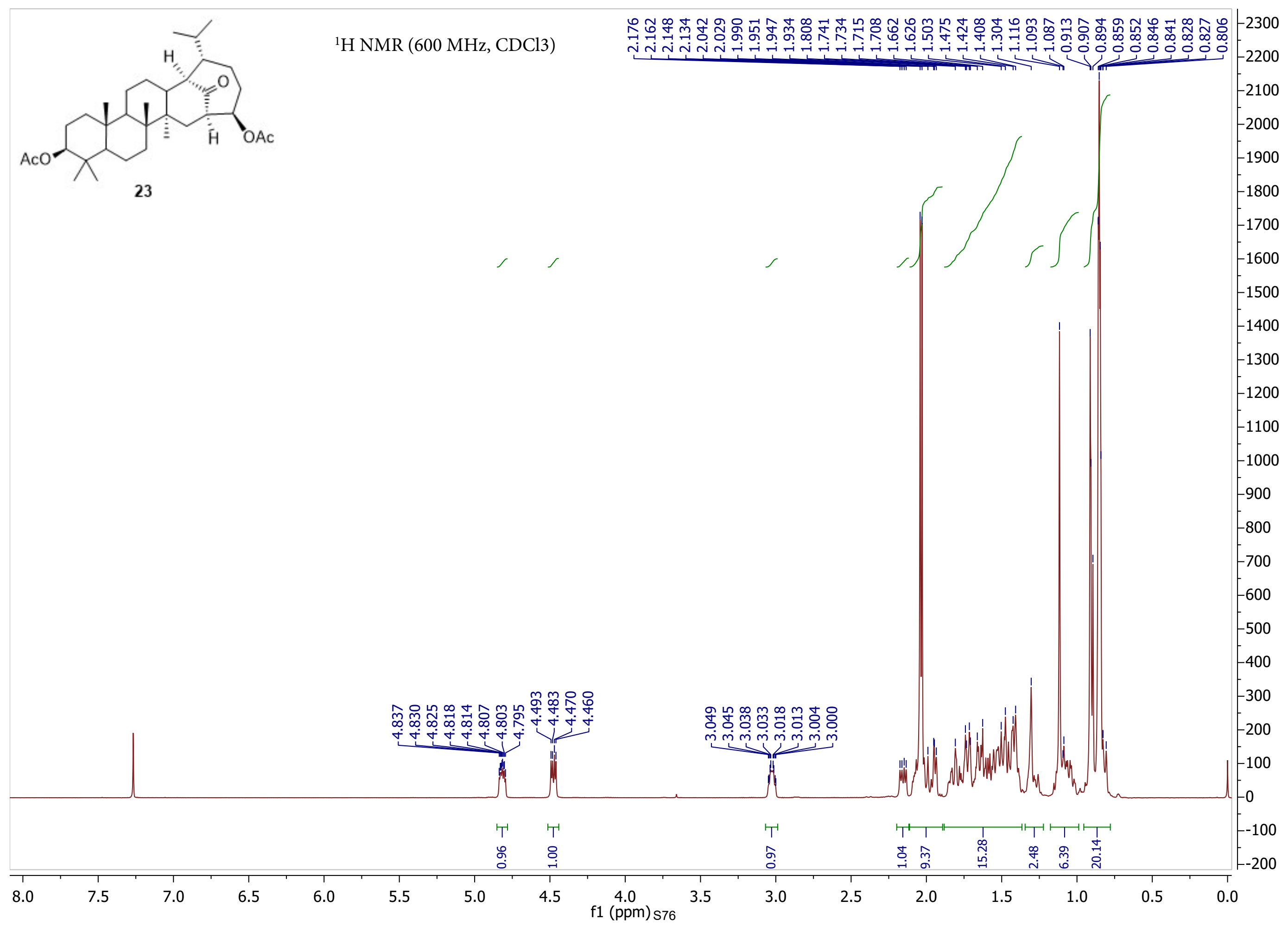




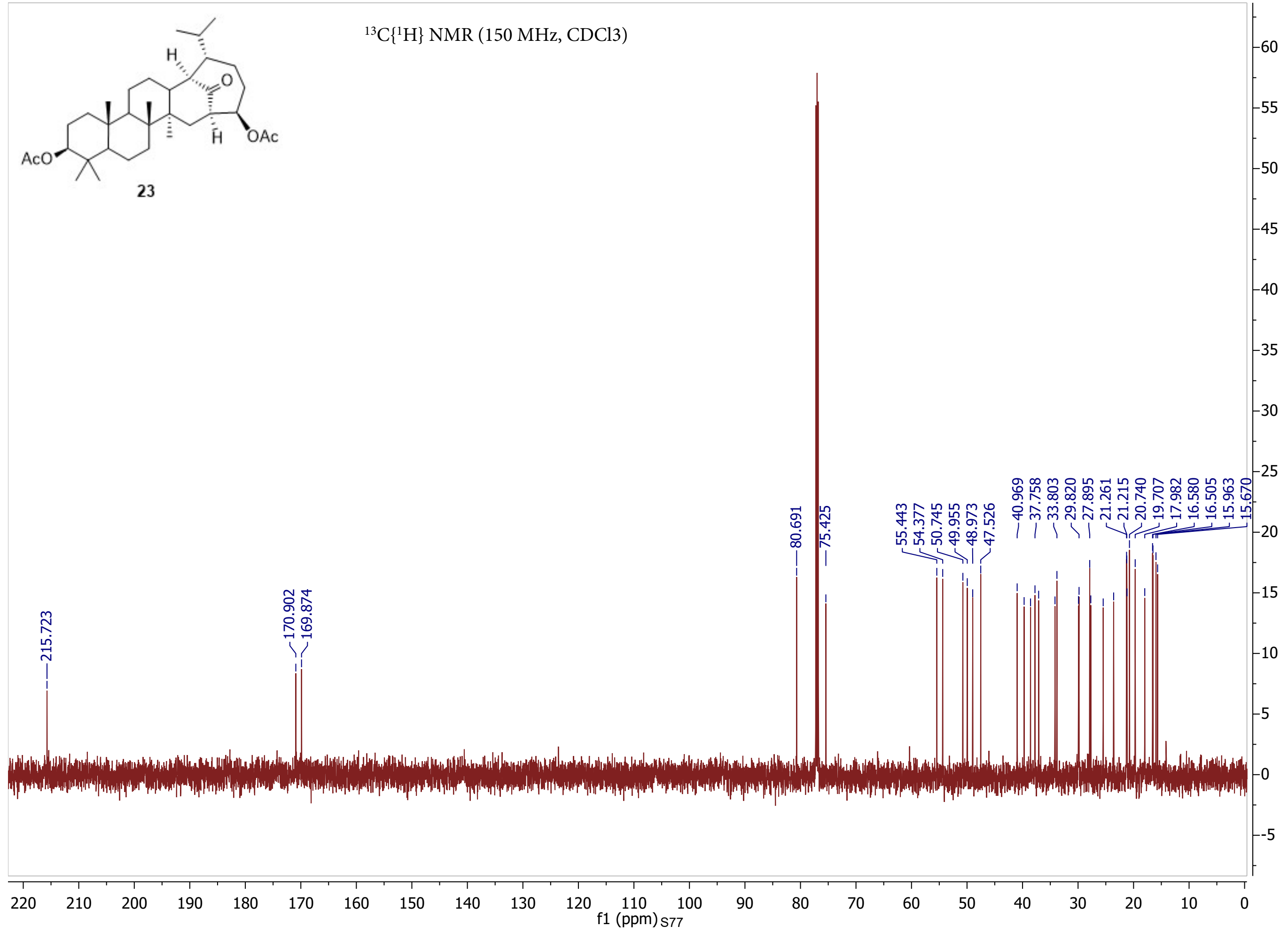




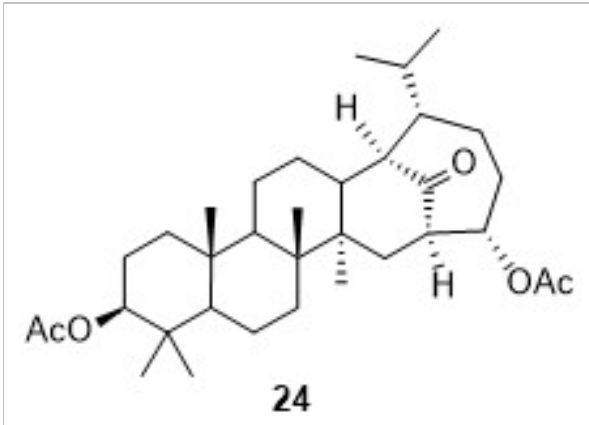

${ }^{1} \mathrm{H}$ NMR (600 MHz, CDCl3)

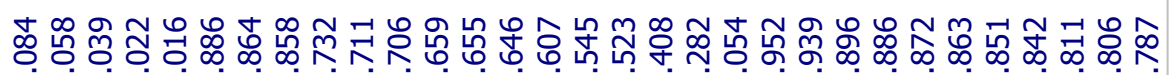

$-1300$
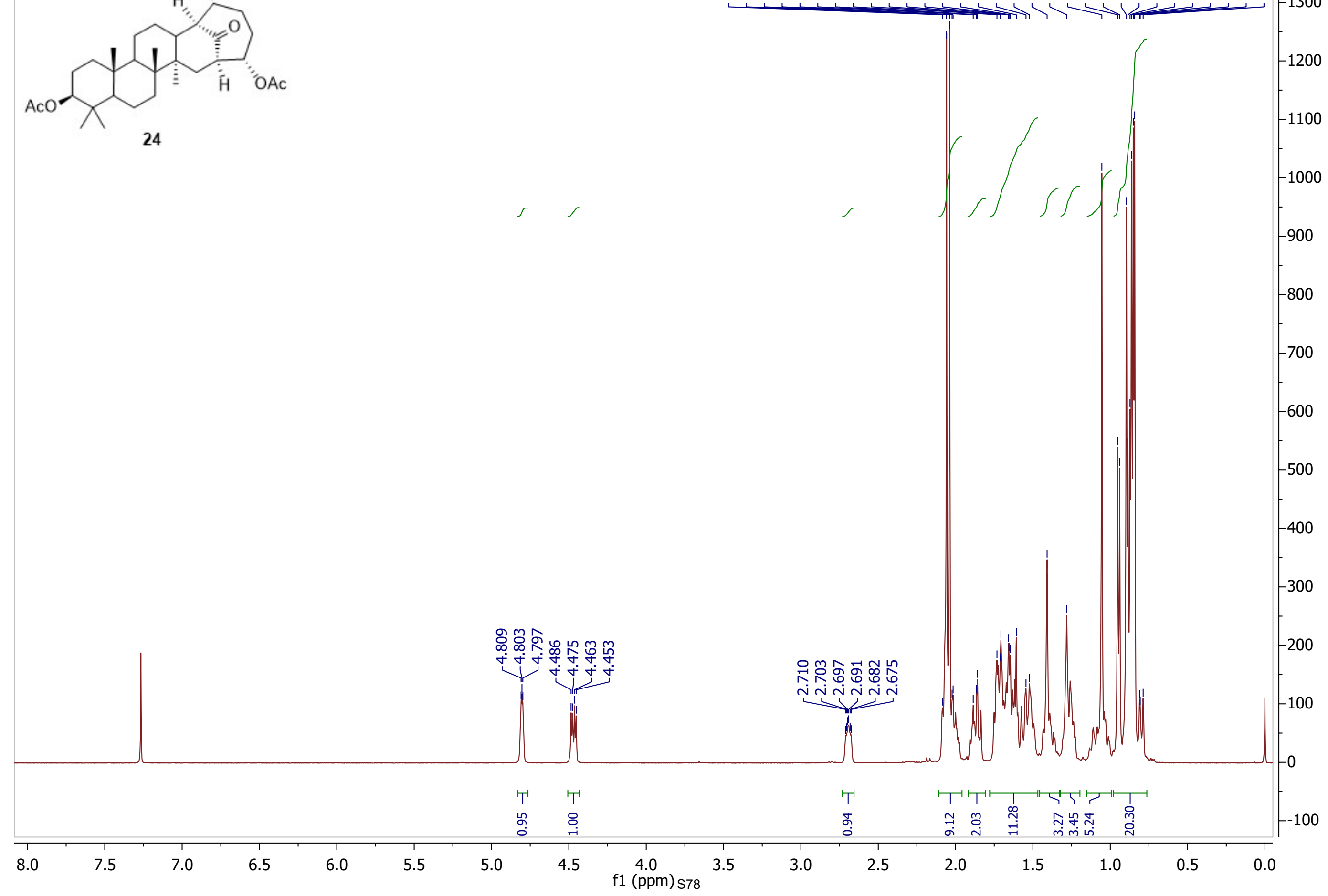


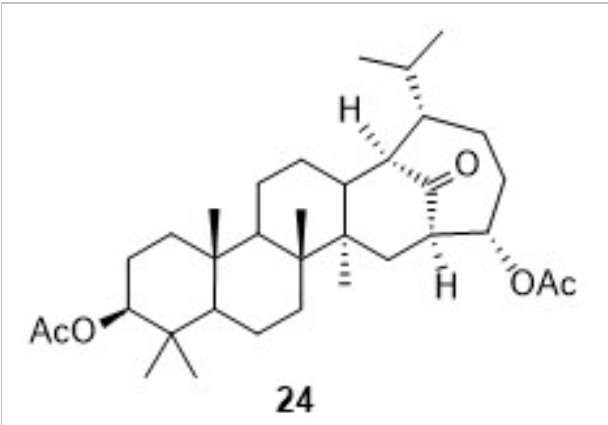

${ }^{13} \mathrm{C}\left\{{ }^{1} \mathrm{H}\right\}$ NMR $(150 \mathrm{MHz}, \mathrm{CDCl} 3)$ 